\title{
Joint Routing, Scheduling and Power Allocation in Generic Multihop Wireless Networks
}

\section{Rozita Rashtchi}

A thesis submitted to the Faculty of Graduate and Postdoctoral

Affairs in partial fulfillment of the requirements

for the degree of

\section{Doctor of Philosophy}

in

Electrical and Computer Engineering

\author{
Carleton University \\ Ottawa, Ontario, Canada \\ January, 2016
}

(C)2016- Rozita Rashtchi 
The undersigned hereby recommends to the

Faculty of Graduate and Postdoctoral Affairs acceptance of the thesis

\title{
Joint Routing, Scheduling and Power Allocation in Generic Multihop Wireless Networks
}

\author{
submitted by Rozita Rashtchi \\ in partial fulfillment of the requirements for the degree of \\ Doctor of Philosophy
}

Professor Ben Liang, External Examiner

Professor Halim Yanikomeroglu, Thesis Co-supervisor

Professor Ramy Gohary, Thesis Co-supervisor

Professor Yvan Labiche, Department Chair

Carleton University

2016 


\section{Abstract}

Future wireless networks are expected to be OFDMA-based with generic topologies in the sense that nodes in addition to being a source and a destination, can act as a half-duplex relay. We consider a joint design that incorporates the physical, medium access and network layers of a network. The goal is to determine the data routes, subcarrier schedules, and power allocations that maximize a weighted sum of the rates, reliably communicated over the network. Four instances are considered.

In the first instance, subcarriers are allowed to be time-shared by multiple links in the network. We cast this problem in an efficiently solvable convex form.

In the second instance, time-sharing is not allowed, and each subcarrier is exclusively assigned to one link throughout the signalling interval. The joint design in this case results in a mixed integer programming. Developing insight into this problem, we propose lower and upper bounds on the weighted-sum rate of the network.

In the third instance, the network employs frequency-reuse, whereby a subchannel might be used simultaneously by multiple nodes. In this case, the joint design problem is nonconvex, and hence to circumvent this difficulty, an efficient technique based on geometric programming is developed to obtain a sub-optimal solution.

In the last instance, each subcarrier can be reused and time-shared by multiple links, thereby generalizing the first three instances and can therefore offer significant performance gains over them. We develop a framework to cast the joint design problem in an optimization form which gives rise to a nonconvex optimization problem. Using approximation techniques based on geometric programming, we provide a computationally-efficient method for obtaining locally optimal solutions.

The generalized design is appealing for designing of future networks due to potentially higher gains. However, this gain comes at the price of complexity which grows exponentially with the size of the network. To circumvent this difficulty, we develop an iterative two-stage algorithm which we refer to it as constraint-splitting and it exhibits fast convergence and finds a sub-optimal power allocation in polynomial time. 
To whom this thesis matters most, My parents, Molouk and Alireza, and my husband, Mehdi. 


\section{Acknowledgments}

It is a pleasure to express my gratitude to many people who directly and indirectly helped and made this thesis possible.

First and foremost, I am greatly indebted to my supervisor, Professor Halim Yanikomeroglu, for his invaluable guidance, endless support and precious patience. Most notably, I would like to thank him for his tremendous efforts to build and continuously expand such a dynamic and large research group. It is never an overstatement to say that one simply could not wish for a better supervisor. My sincere thanks go to my co-supervisor, Dr. Ramy Gohary, for his generous support along the journey of my $\mathrm{PhD}$. He patiently taught me a great deal of optimization and technical writing. Through many late day discussions his insightful advice and questions inspired me to widen and deepen my research in various perspectives. Many thanks go to all the members of our wonderful research group for their encouragement during the course of this research.

A special gratitude and love goes to my parents, Molouk and Alireza, who always supported me and gave me the courage to follow my dreams. I am also very grateful to my sister and brother, Ramina and Amir, who always stood by me with smile and good wishes. My deepest gratitude is reserved for my lovingly husband, Mehdi. Without his tolerance, patience and unfailing support, completing this thesis would not have been possible. 


\section{Table of Contents}

Abstract $\quad$ iii

Acknowledgments $\quad$ V

Table of Contents vi vi

List of Tables $\quad$ X

List of Figures $\quad$ xi

List of Symbols $\quad$ xiii

List of Acronyms $\quad$ XV

1 Introduction 1

1.1 Motivation . . . . . . . . . . . . . . . . . . . . . 1

1.2 Problem Statement . . . . . . . . . . . . . . . . . . . 3

1.3 Contributions .......................... 5

1.3.1 JRSPA with Time-sharing and without Frequency-reuse . . . 5

1.3.2 JRSPA without Time-sharing and without Frequency-reuse . . 6

1.3.3 JRSPA without Time-sharing and with Frequency-reuse . . . 7

1.3.4 JRSPA with Time-sharing and with Frequency-reuse . . . . . 7

1.3.5 Generalized JRSPA: A Low Complexity Approach . . . . . . . 8

1.4 List of Publication . . . . . . . . . . . . . . . . . . . . . . . 9

1.4.1 Published Journal Papers . . . . . . . . . . . . . . . . . 9

1.4.2 Published Conference Papers . . . . . . . . . . . . . . . 9

1.4 .3 Journal Manuscripts . . . . . . . . . . . . . . . . . . . 10

1.5 Organization of the Thesis . . . . . . . . . . . . . . 10 
2 Literature Review 11

2.1 Introduction . . . . . . . . . . . . . . . . . . . . 11

2.2 Power Allocation in Single-carrier Networks . . . . . . . . . . . . 12

2.3 Joint Scheduling and Power Allocation in OFDMA Networks . . . . . 12

2.4 Routing in Multihop Relay Networks . . . . . . . . . . . . . . 14

2.5 JRSPA in Relay Networks . . . . . . . . . . . . . . . . . 14

2.6 JRSPA in Generic OFDMA Networks . . . . . . . . . . . . . 15

3 System Model and Preliminaries 16

3.1 Introduction . . . . . . . . . . . . . . . . . . . 16

3.2 Network Model . . . . . . . . . . . . . . . . . . . . . . . . . 16

3.3 Communication Model . . . . . . . . . . . . . . . . . . . . 17

3.4 Generic Network . . . . . . . . . . . . . . . . . . . . 18

3.5 Design Variables . . . . . . . . . . . . . . . . . . . . 22

3.5.1 Routing Variables . . . . . . . . . . . . . . . . . . . . 22

3.5.2 Power Allocation Variables . . . . . . . . . . . . . . . . . 22

3.5.3 Scheduling Variables . . . . . . . . . . . . . . . . . . 24

3.6 Design Objective . . . . . . . . . . . . . . . . . . . . . . . . . 24

3.7 Implementation $\ldots \ldots \ldots \ldots \ldots \ldots \ldots \ldots \ldots \ldots$

4 JRSPA with Time-Sharing and without Frequency-Reuse 27

4.1 Introduction . . . . . . . . . . . . . . . . . . . . . . 27

4.2 System Constraints . . . . . . . . . . . . . . . . . . . . . . 27

4.2 .1 Routing Constraints . . . . . . . . . . . . . . . 27

$4.2 .2 \quad$ Scheduling Constraints . . . . . . . . . . . . . . . 28

4.2 .3 Power Allocation Constraints . . . . . . . . . . . . . . . . 29

4.2.4 Capacity Constraints . . . . . . . . . . . . . . . . . . . 29

4.3 Problem Formulation . . . . . . . . . . . . . . . . . . 30

4.4 The Optimal Solution . . . . . . . . . . . . . . . . . . . 31

4.5 Complexity Analysis . . . . . . . . . . . . . . . . . 32

4.6 Numerical Results . . . . . . . . . . . . . . . . . . . . . . . . 33

4.7 Conclusion . . . . . . . . . . . . . . . . . . . . . . . . . . . . . 36

5 JRSPA without Time-Sharing and without Frequency-Reuse $\quad 38$

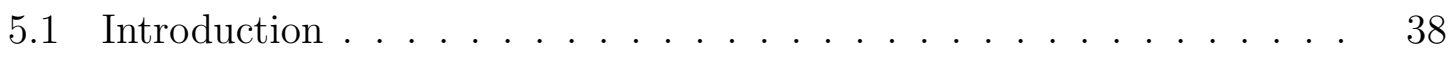


5.2 System Constraints . . . . . . . . . . . . . . . . . . . . 38

5.3 Problem Formulation . . . . . . . . . . . . . . . . . . . . . . . . . 39

5.4 Proposed Lower Bounds . . . . . . . . . . . . . . . . . . . 40

5.4 .1 The Rounding-Based Approach . . . . . . . . . . . . . 40

5.4 .2 The GP-based Approach . . . . . . . . . . . . . . . . 41

5.5 Complexity Analysis . . . . . . . . . . . . . . . 45

5.6 Numerical Results . . . . . . . . . . . . . . . . . . . . . . . . 46

5.7 Conclusion . . . . . . . . . . . . . . . . . . . . . . . . . . . . 52

6 JRSPA without Time-Sharing and with Frequency-Reuse 53

6.1 Introduction . . . . . . . . . . . . . . . 53

6.2 System Constraints . . . . . . . . . . . . . . . . . . 53

6.2 .1 Routing Constraints . . . . . . . . . . . . . . . 53

6.2.2 Power Allocation Constraints . . . . . . . . . . . . . 54

6.2 .3 Capacity Constraints . . . . . . . . . . . . . . 55

6.3 Problem Formulation . . . . . . . . . . . . . . . . . . . 55

6.4 Proposed GP-based Algorithm . . . . . . . . . . . . . . . 56

6.5 Complexity Analysis . . . . . . . . . . . . . . . . 58

6.6 Numerical Results . . . . . . . . . . . . . . . . . . . . . . . . . . . 59

6.7 Conclusion . . . . . . . . . . . . . . . . . . . . . 64

7 JRSPA with Time-Sharing and with Frequency-Reuse $\quad 66$

7.1 Introduction . . . . . . . . . . . . . . . . 66

7.2 System Constraints . . . . . . . . . . . . . . . . 66

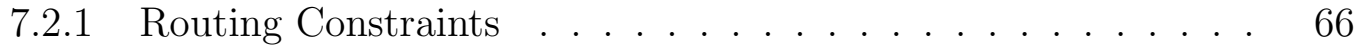

7.2 .2 Scheduling Constraints . . . . . . . . . . . . 67

7.2 .3 Power Allocation Constraints . . . . . . . . . . . . . . 69

7.2 .4 Capacity Constraints . . . . . . . . . . . . . . . 70

7.3 Problem Formulation . . . . . . . . . . . . . . . . . 70

7.4 Proposed GP-based Algorithm . . . . . . . . . . . . . . 71

7.5 Complexity Analysis . . . . . . . . . . . . . . . . 73

7.6 Numerical Results . . . . . . . . . . . . . . . . . . . . . . . . . . 74

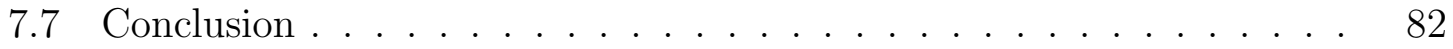


8 Generalized JRSPA: A Low-Complexity Approach 85

8.1 Introduction . . . . . . . . . . . . . . . . . . . 85

8.2 Network Analysis . . . . . . . . . . . . . . . . . . . . 85

8.3 Problem Formulation . . . . . . . . . . . . . . . . . . . . 87

8.4 Joint Design Sub-problems: Scheduling and Power Allocation . . . . . 88

8.4.1 Scheduling with Fixed Power Allocations . . . . . . . . . . . 88

8.4.2 Power Allocation with Fixed Schedules . . . . . . . . . . . . . 89

8.5 Proposed Low Complexity Approach . . . . . . . . . . . . . . . . . . 93

8.6 Complexity Analysis . . . . . . . . . . . . . . . . . . . . . 96

8.6.1 Computational complexity of the first stage . . . . . . . . . . 96

8.6.2 Computational complexity of the second stage . . . . . . . . 97

8.6.3 Computational complexity of the two-stage approach . . . . . 97

8.7 Numerical Results . . . . . . . . . . . . . . . . . . . . . 98

8.8 Conclusion . . . . . . . . . . . . . . . . . 102

9 Conclusions and Future Work 104

9.1 Summary and Contributions . . . . . . . . . . . . . . . . . 104

9.2 Future Work . . . . . . . . . . . . . . . 106

A Geometric Programming 109

A.1 The GP Standard Form . . . . . . . . . . . . . . . . . . 109

A.2 Monomial Approximation . . . . . . . . . . . . . . . 109

B Single Condensation Method 110

C Complexity Analysis 111

C.1 Self-Concordance . . . . . . . . . . . . . . . . . . 111

C.2 Proof of Proposition $1 \ldots \ldots \ldots \ldots 11$

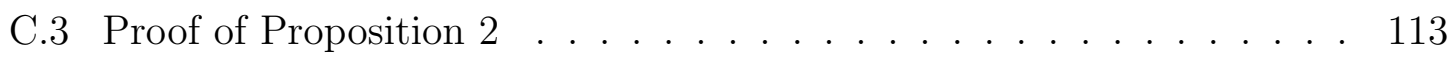

C.3.1 Proving the First Statement of Proposition 2 . . . . . . . . . . 113

C.3.2 Proving the Second Statement of Proposition 2 . . . . . . . . 113

C.3.3 Proving the Third Statement of Proposition 2 . . . . . . . . . 115

C.4 Proof of Proposition $3 \ldots \ldots \ldots$

C.5 Proof of Proposition 4 . . . . . . . . . . . . . . . . . . . . . . . 115

$\begin{array}{ll}\text { List of References } & 115\end{array}$ 


\section{List of Tables}

2.1 Related Work . . . . . . . . . . . . . . . . . . . 11

5.1 Channel gains $[\mathrm{dB}]$ in Example $3 \ldots \ldots \ldots \ldots \ldots$

6.1 Power allocations $(\mathrm{mW})$ in Example $1 . \ldots \ldots \ldots \ldots$

6.2 Channel gains in Example 2. . . . . . . . . . . . . . . 61

7.1 Successive GP-based Algorithm for Solving (8.4) . . . . . . . . 73

7.2 Channel gains $[\mathrm{dB}]$ in Example $1 \ldots \ldots \ldots \ldots \ldots$

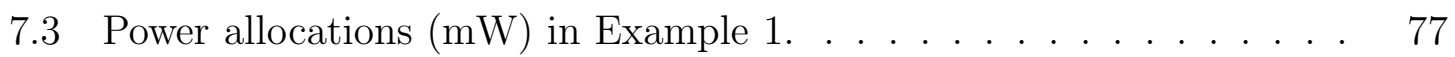




\section{List of Figures}

1.1 An example of future wireless networks. . . . . . . . . . . . . . 2

3.1 A cellular network. . . . . . . . . . . . . . . . . . . . . 19

3.2 A relay-assisted cellular network. . . . . . . . . . . . . . . 20

3.3 A mesh backhaul network. . . . . . . . . . . . . . . . . 21

3.4 A D2D communication network. . . . . . . . . . . . 22

3.5 A network with $N=4, K=2$ (solid and dashed lines) and $D=\{1,2\} .23$

3.6 Scheduling samples for designs (a) w. time-sharing, w/o frequencyreuse, (b) w/o time-sharing, w/o frequency-reuse, (c) w/o timesharing, w. frequency-reuse, and (d) w. time-sharing, w. frequencyreuse. Dark sections represent transmission intervals and light sections represents silent intervals. . . . . . . . . . . . . . . . . . . 24

4.1 Network schematic with $N=3, L=6, K=4$ and $D=1$. . . . . 33

4.2 An achievable rate region for the network shown in Figure 4.1 with the channel coefficients in (4.12). . . . . . . . . . . . . . . 35

4.3 (Example 2) Sum rate generated by the optimal and heuristic JRSPA. 36

5.1 The routes generated by designs considered for the 10-node network in Example 1. . . . . . . . . . . . . . . . . . . . . . . . . . . . . 48

5.2 (Example 1) Sum rate generated by optimal, rounding-based and GPbased JRSPA. . . . . . . . . . . . . . . . . . . . . . . . . . . . . 49

5.3 Topology of the network considered in Example 2. . . . . . . . . . . . 50

5.4 (Example 2) Sum rate generated by upper and lower bounds and optimal binary design. . . . . . . . . . . . . . . 51

5.5 An achievable rate region for the network in Example 3 with the channel gains in Table 5.1. . . . . . . . . . . . . . . . . . . . . 52

6.1 Data routes for (a) $d=1$, (b) $d=2$ in Example 1. . . . . . . . . 60

6.2 Network schematic. Each link is composed of two subcarriers. . . . . 62 
6.3 Sum rate of proposed and continuous scheduling-based designs for the network considered in Example 2. . . . . . . . . . . . . . . . . . . .

6.4 Schematic of the network in Example 3, data routes, subcarriers reuse and corresponding power allocations. . . . . . . . . . . . .

6.5 Sum rate of proposed and continuous scheduling-based designs for the network considered in Example 3. . . . . . . . . . . . . . . . . . . 65

7.1 An exemplary scheduling for a network with $L=3, K=1$. . . . . . 67

7.2 Different ordering of transmissions of the scheduling in Figure 7.1. . . 68

7.3 Data routes for (a) $d=1$, (b) $d=2$ in Example 1. . . . . . . . 76

7.4 Time-sharing schedules of the subcarriers in Example 1. . . . . . . . . 77

7.5 Average weighted-sum rate comparison for (a) $w_{3}^{(2)}=5 w_{4}^{(1)}$, (b) $w_{3}^{(2)}=$

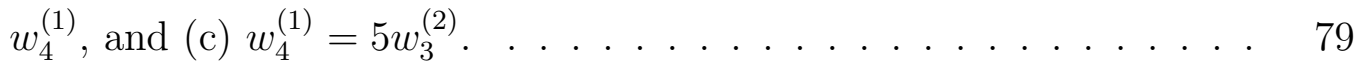

7.6 Sum-rate generated by the generalized algorithm for different values of $I$.

7.7 (a) Convergence behaviour and (b) performance of the generalized algorithm with different initial points. . . . . . . . . . . . . . . . 83

7.8 Rate-region comparison. . . . . . . . . . . . . . . . . . . 84

7.9 Average weighted-sum rate comparison. . . . . . . . . . . . . . . 84

8.1 The scheduling matrix for a 3-node network with $L=6$ links. . . . . 87

8.2 Block diagram of the proposed algorithm in Algorithm 2 . . . . . . . 96

8.3 Network topology . . . . . . . . . . . . . . . . . . . . . . . . 99

8.4 Performance evaluation . . . . . . . . . . . . . . . . . . 100

8.5 Convergence behaviour . . . . . . . . . . . . . . . . . . 101

8.6 Performance comparison . . . . . . . . . . . . . . . . . . 102 


\section{List of Symbols}

\begin{tabular}{ll}
\hline Symbol & Definition \\
\hline \hline$N$ & Number of nodes \\
$L$ & Number of links \\
$K$ & Number of subcarriers \\
$D$ & Number of destinations \\
$W$ & The bandwidth of each subcarrier \\
$\mathcal{N}$ & The set of all nodes \\
$\mathcal{L}$ & the set of all links \\
$\mathcal{K}$ & The set of all subcarriers \\
$\mathcal{O}(n)$ & The set of outgoing links from node $n$ \\
$\mathcal{I}(n)$ & The set of incoming links to node $n$ \\
$a_{n \ell}$ & Node-link connection indicator \\
$a_{n \ell}^{+}$ & Outgoing link indicator at node $n$, i.e., $a_{n \ell}^{+}=\max \left\{0, a_{n \ell}\right\}$ \\
$a_{n \ell}^{-}$ & Incoming link indicator at node $n$, i.e., $a_{n \ell}^{-}=\left|\min \left\{0, a_{n \ell}\right\}\right|$ \\
$h_{n n^{\prime}}^{(k)}$ & Channel coefficient on subcarrier $k$ of the link connecting node $n$ \\
& to node $n^{\prime}$ \\
$g_{\ell k}$ & Normalized channel gain on the subcarrier $k$ of link $\ell$ \\
$u_{n}^{(k)}$ & The signal transmitted by node $n$ on subcarrier $k$ \\
$y_{n}^{(k)}$ & The signal received by node $n$ on subcarrier $k$ \\
\hline
\end{tabular}




\begin{tabular}{ll}
\hline Symbol & Definition \\
\hline \hline$v_{n}^{(k)}$ & The zero-mean additive Gaussian noise on subcarrier $k$ at node $n$ \\
$N_{0}$ & Noise variance \\
$s_{n}^{(d)}$ & The rate of data injected into node $n$ and intended for destination $d$ \\
$x_{\ell k}^{(d)}$ & The rate of data on subcarrier $k$ of link $\ell$, intended for destination $d$ \\
$p_{n}^{(k)}$ & The power emitted by node $n$ on subcarrier $k$ \\
$q_{\ell k}$ & The power allocated to subcarrier $k$ of link $\ell$ \\
$w_{n}^{(d)}$ & The weight assigned to the rate $s_{n}^{(d)}$ \\
$c_{\ell k}$ & The fraction of time in which subcarrier $k$ of link $\ell$ is scheduled for \\
$t_{\ell k}^{(d)}$ & transmission \\
$\gamma_{\ell_{1} \ldots \ell_{m}}^{(k)}$ & The fraction of time in which subcarrier $k$ in used by links $\ell_{1}, \cdots, \ell_{m}$ \\
& simultaneously \\
$y_{\ell k}$ & The set of all scheduling variables \\
& The total power budget at node $n$
\end{tabular}




\section{List of Acronyms}

\begin{tabular}{ll}
\hline Acronym & Definition \\
\hline \hline $4 \mathrm{G}$ & Fourth Generation \\
BS & Base Station \\
CDMA & Code Division Multiple Access \\
CSI & Channel State Information \\
D2D & Device-to-Device \\
FDMA & Frequency Division Multiple Access \\
GP & Geometric Programming \\
IMT-Advanced & International Mobile Telecommunications-Advanced \\
InH & Indoor HotSpot \\
IPM & Interior Point Method \\
JRSPA & Joint Routing, Scheduling and Power Allocation \\
KKT & Karush-Kuhn-Tucker conditions \\
LP & Linear Programming \\
LTE & Long-Term Evolution \\
MAC & Medium Access Control \\
Mbps & Mega bits per second \\
NLoS & Non Line-of-Sight \\
OFDM & Orthogonal Frequency Division Multiplexing \\
\hline
\end{tabular}




\begin{tabular}{ll}
\hline Acronym & Definition \\
\hline \hline OFDMA & Orthogonal Frequency Division Multiple Access \\
QoS & Quality of Service \\
SINR & Signal-to-Interference-plus-Noise Ratio \\
SNR & Signal-to-Noise Ratio \\
UMa & Urban Macro-cell \\
UT & User Terminal \\
\hline
\end{tabular}




\section{Chapter 1}

\section{Introduction}

\subsection{Motivation}

Wireless communication gained commercial momentum during the 1990s with the introduction of cellular networks as a convenient means for voice communication. The primary usage of these networks has since shifted towards data communication, covering applications that range from entertainment-centric to military-centric ones. These data-intensive applications have become an integral part of our daily lives and is expected to increase significantly in the future. In particular, with the introduction of a myriad of smart devices, user demands for high data-rate services such as video streaming and multimedia file sharing, are undergoing an unprecedented rise. According to a recent mobile traffic forecast report by Cisco, the demand for high data-rate services is expected to increase 10-fold by 2019 as compared to 2014 [1]. On the other hand, future wireless networks are envisioned to provide ubiquitous high data-rate coverage for anything and everywhere [2]. However, such a substantial increase in mobile data traffic presents a tremendous challenge in designing future wireless networks, which is due mainly to the scarcity of radio resources. Providing such high data-rates is beyond the limits of current wireless networks and hence, the next generation of wireless networks requires novel techniques.

In conventional wireless networks a geographical area is tessellated into smaller regions called cells and a base station (BS), located in each cell, provides wireless services to mobile user terminals (UTs) in its coverage region. In such traditional networks, the roles of the nodes have been restricted to being either sources or destinations; they did not help each other in relaying information. Meeting the prospective 
increase in demands on the high data-rate services delivered by future wireless networks will bank on the versatility and the portability of the communication devices that will form the nodes of future wireless networks. These devices will be able to perform multiple tasks simultaneously and adapt to varying channel conditions and dynamic network topologies [3]. For instance, the nodes are expected to be capable of sending, receiving and relaying data to other nodes [4]. As such, future communication networks are expected to be less structured and more responsive to instantaneous demands. An example of such networks is depicted in Figure 1.1. In this figure, nodes of the networks help each other in terms of relaying information to other nodes. The dynamic nature of these networks gives rise to a generic topol-

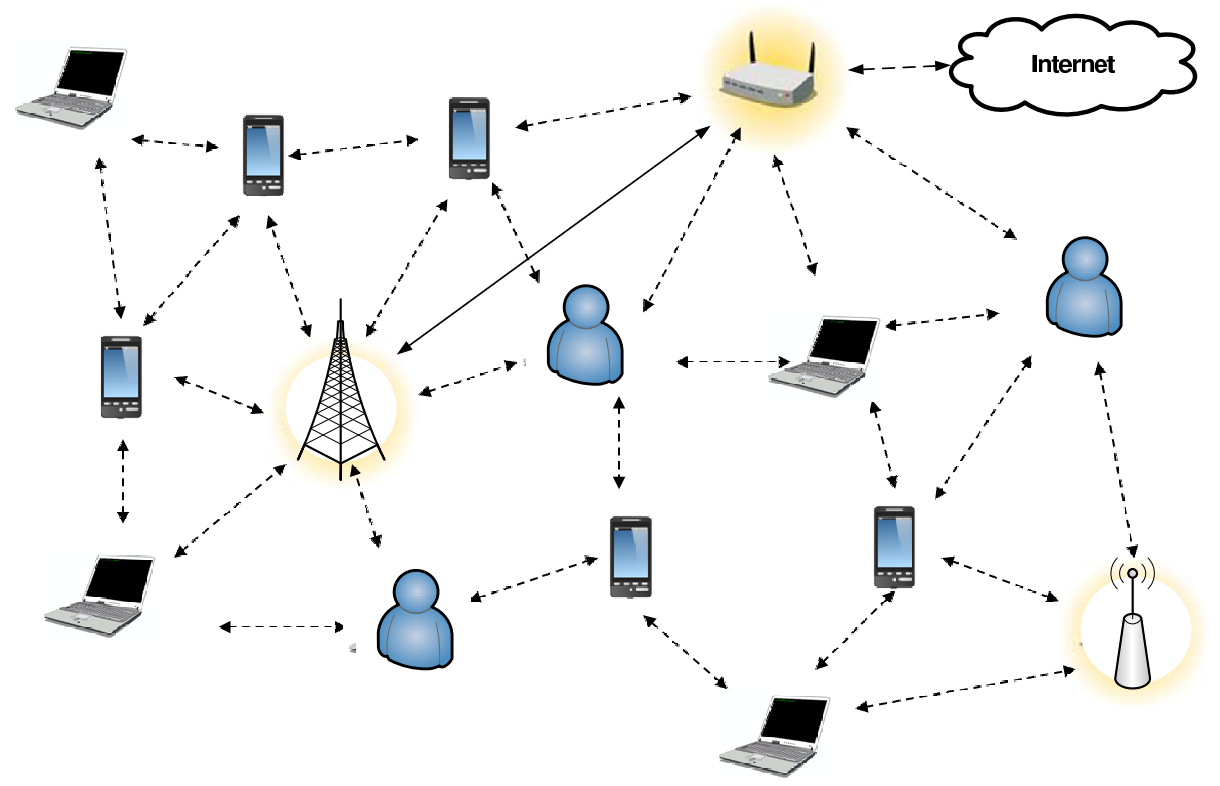

Figure 1.1: An example of future wireless networks.

ogy which encompasses various communication network structures, including current cellular ones and device-to-device (D2D) communication networks [5].

To facilitate the implementation of future wireless networks, these networks are likely to rely on orthogonal frequency division multiple access (OFDMA) for accessing the wireless medium. In particular, OFDMA divides the frequency-selective wideband channel into a set of orthogonal narrowband subcarriers and offers several practical advantages over other multiple access techniques. These advantages include design simplicity and resilience to multiuser interference and frequency-selective fading [6]. In addition, OFDMA offers an effective means for sharing the frequency band between 
multiple terminals, depending on their channel conditions [7]. Furthermore, by providing multiple orthogonal channels between any two nodes in the network, OFDMA exposes the degrees of freedom inherent in the system design. Additionally, OFDMA provides an opportunity for flexible routing by offering multiple path options to route the traffic between sources and destinations.

Another feature of future wireless networks is that the resources available for their operation are likely to be rather scarce. Such resources include the available frequency spectrum, time and the typically low power of the batteries to be used by the wireless terminals. Given the scarcity of these resources, accommodating high data-rate services imposes stringent constraints on the way the communication resources are exploited. This leaves little room for resources to be wasted. To properly exploit these resources, the routes taken by each data stream must be carefully selected, the subcarriers used for transmissions must be properly scheduled, and the powers allocated to these transmissions must be judiciously determined. In particular, although the isolated design of system functionalities, e.g., routing and power allocation, simplifies the design of the communication network, these functionalities are interrelated and considering them conjointly results in more effective utilization of the available resources. A practical impediment is the computational burden incurred by the joint consideration of these functionalities. This is especially true for networks with a large number of wireless terminals with various capabilities. Hence, it is desirable to develop joint designs that are not only close to being optimal, but also efficiently computable. This is the main focus of this thesis.

\subsection{Problem Statement}

To avoid the performance loss resulting from the separate design of various tasks, in this thesis we devise optimization frameworks that incorporate joint routing, scheduling and power allocation (JRSPA) in the design of a generic multicarrier network. The objective of the design is to maximize a weighted-sum of the rates injected and reliably communicated over the network. Weights are assumed to be known a priori, but can be adapted over time to account for fairness issues and to maintain a desired quality of service. The nodes can assume multiple roles simultaneously including being sources, destinations and/or relays. The nodes acting as relays are restricted to operate in the half-duplex mode, i.e., a node cannot send and receive at 
the same time on the same subcarrier.

Given the stringent limitations on the spectrum available for wireless communications, providing high data-rate services banks on sharing the spectrum by multiple users which results in potentially significant interference. Cross-layer designs can be classified according to whether the available subcarriers can be reused by multiple nodes simultaneously and whether these subcarriers can be time-shared by them.

Designs with time-sharing and without frequency-reuse To facilitate the design of OFDMA-based wireless networks, each subcarrier can be restricted to be exclusively used at any given instant by one of the nodes in the network. Such scheduling ensures that the transmissions of the nodes do not interfere with each other.

Designs without time-sharing and without frequency-reuse In many practical scenarios, allowing the subcarriers to be time-shared by multiple terminals increases the overhead required for establishing coherent communication between the wireless terminals. This is especially true in networks with time-varying channels. In these situations, it is more practical to restrict the subcarrier schedules to be used exclusively by one terminal. Such schedules result in assigning each subcarrier to a particular node for the entire signalling interval.

Designs without time-sharing and with frequency-reuse Despite the practical advantages of using subcarrier scheduling for avoiding interference, the rates provided by the networks that use this technique may be significantly inferior to those provided by networks that allow the subcarriers to be used simultaneously by multiple nodes. This is especially the case when the network is composed of essentially separated clusters; subcarrier reuse is optimal for clustered networks. In this scenario, a subcarrier, once assigned to a set of nodes, will be used by those nodes throughout the signalling interval.

Designs with time-sharing and with frequency-reuse Although using a subcarrier exclusively by one node (i.e., without reuse) throughout the signalling interval (i.e., without time-sharing) is relatively easy to implement in practice, this approach limits the network capabilities and deprives it from achieving rates that can be reliably communicated over it. Networks in which subcarriers are allowed to be reused 
and time-shared jointly enhance the network capabilities and support data-rates well beyond those supported by networks that consider frequency-reuse and time-sharing separately. This scenario subsumes other ones as special cases and hence, can be considered as a generalization of them.

The latter instance generalizes the other three, and can hence provide a superior performance compared to them. This makes it appealing for designing future wireless networks; however, this performance gain comes at the price of increased complexity. In fact, this design offers a tradeoff between performance gain and computational complexity; the complexity of the design grows fast with the size of the network which makes it suitable for designing small-to-medium size networks.

With the rapid growth of wireless devices, future wireless networks are expected to be denser with massive connectivity. Hence, it is desirable to develop techniques that not only provide higher gains, but also have low computational costs even for large size networks. Developing such techniques is the main focus of this thesis.

\subsection{Contributions}

In this thesis, we consider the JRSPA problem for the four instances of the network described in Section 1.2. For these networks, it is assumed that the design problem is solved by a central entity that has access to the channel state information (CSI) and that provides the nodes with the signalling parameters. The main contributions of this thesis are summarized below.

\subsubsection{JRSPA with Time-sharing and without Frequency- reuse}

In this instance, each subcarrier is scheduled to be used exclusively by one node at any given time instant. In this case, the scheduling variables assume continuous values, and the design problem is referred to as "continuous JRSPA".

By changing the design variables, this problem is cast in a convex form that can be solved efficiently to yield the set of all the rates that using this approach can be reliably communicated over a given OFDMA-based wireless network, and the data routes, the subcarrier schedules and the power allocations that achieve them. This

form is amenable to efficient interior point method (IPM)-based solvers and enables 
us to efficiently determine a set of all the rates that are achievable in a given network using this approach.

Numerical results show that networks that utilize time-sharing can achieve significantly higher rates than networks in which time-sharing is not allowed, and that the advantage of time-sharing becomes more pronounced as the network size increases.

\subsubsection{JRSPA without Time-sharing and without Frequency- reuse}

In this instance, time-sharing is not allowed, and each subcarrier is exclusively assigned to one link throughout the signalling interval. Thereby, the scheduling variables assume binary values, and result in a complex mixed integer JRSPA problem [7], which we refer to as "binary JRSPA". To alleviate the difficulty that arises in this case, we develop bounds on the rates that can be achieved by this network.

Rounding-based Lower Bound First we note that, because continuous scheduling is a relaxation of binary scheduling, the weighted-sum rates it achieves constitute an upper bound on the weighted-sum rates achieved by its binary counterpart. To develop lower bounds, we observe that normalizing and rounding the (optimal) continuous schedules obtained in the case of continuous scheduling yields, potentially suboptimal, binary schedules. With these schedules fixed, the JRSPA design problem can be cast in a convex form and this form is used to obtain the first lower bound.

GP-based Lower Bound To develop another lower bound, the restriction of the schedules to be binary is captured by imposing a set of constraints on the power allocations. The resulting JRSPA design problem is nonconvex. To overcome this difficulty, a logarithmic transformation is used to cast the original problem in a form that, for all but a few constraints, complies with the geometric programming (GP) [8] standard form. The constraints that are not compatible with that form are then approximated by monomial expressions corresponding to their first order Taylor series expansion around a given initial point [9]. Using an exponential transformation, the resulting approximation can be cast in the form of a convex optimization problem. A refinement of this approximation can be obtained by iterative updating of the initial point. In particular, we use the so-called iterative monomial approximation technique, 
wherein the solution of one convex approximation is used as the initial point in the following iteration. One of the advantages of this technique is that, under relatively mild conditions, it is guaranteed to yield a solution of the Karush-Kuhn-Tucker (KKT) system that corresponds to the original optimization problem [10]. This technique yields locally optimal routes and power allocations, which are subsequently used to recover the binary schedules.

When time-sharing is not allowed, numerical results suggest that the gap between the lower bounds and the true maximum is usually small, specially at low SNRs.

\subsubsection{JRSPA without Time-sharing and with Frequency- reuse}

In this instance time-sharing is not allowed and a subcarrier, once assigned to a set of links, will be used by those links throughout the signalling interval. This case results in binary subcarrier scheduling variables with frequency-reuse, which are implicitly captured in our formulations by the power allocation design variables.

Unfortunately, the optimization problem arising from the joint design in this case is nonconvex and hence, difficult to solve. By manipulating the constraints, we cast the JRSPA design problem is a form that is amenable to an efficient GP-based approximation technique, analogous to the one proposed for the case in Section 1.3.2.

Numerical results show that, in some scenarios, the design developed for this case yields achievable data-rates that are superior to those yielded by designs in which the subcarriers are not reused by multiple links.

\subsubsection{JRSPA with Time-sharing and with Frequency-reuse}

In contrast to the previous instances, in this instance, each subcarrier can be both reused and time-shared by multiple links. This results in continuous subcarrier scheduling variables with frequency-reuse. Mathematical manipulation of these variables constitutes the intricate task of determining the number of times and the duration over which a particular subcarrier is reused. It is worth noting that this case is a generalization of the three previous cases. As such, the framework considered in

this case can offer significant performance advantages over other designs, but at the expense of increasing dimensionality and design complexity. In fact, the design in this case provides an inherent tradeoff between performance and design complexity; the 
design complexity can be reduced by restricting the number of times that a particular subcarrier can be reused.

Numerical results show that the designs developed in this instance yields performance that is considerably superior to that of their counterparts in the previous instances.

\subsubsection{Generalized JRSPA: A Low Complexity Approach}

The problem we considered in this instance is the same as the one considered in Section 1.3.4, i.e., generalized JRSPA. The approach we took there to find a solution for this problem was based on GP and monomial approximations. This approach solved the problem but at the expense of exponential complexity and slow convergence which makes that approach suitable for designing small-to-medium size networks. In contrast, in this section our goal is to develop a low-complexity algorithm that exhibits fast convergence for larger networks. Our approach is to decompose the problem into two smaller but tractable sub-problems, i.e., scheduling and power allocation. These sub-problems are solved iteratively, i.e., the output of one sub-problem is used to obtain an initial point for the other sub-problem in the subsequent iteration.

Scheduling Sub-problem In the first stage, we solve the design sub-problem when power allocations are fixed. This assumption yields an efficiently solvable Linear Program (LP).

Power Allocation Sub-problem In the second stage, we consider the design problem when subcarrier schedules are fixed. This problem is nonconvex and difficult to solve. To overcome this difficulty we develop a novel iterative technique that we refer to as constraint-splitting. This technique exhibits fast convergence and finds a suboptimal power allocation in polynomial time. The philosophy of this technique is to split a particular constraint into two parts. We observe that by fixing the right-handside (RHS) of that constraint and defining an appropriate lower bound, the problem can be cast as a GP, which can be readily converted into a convex optimization problem. We also observe that fixing the left-hand-side (LHS) of that constraint makes the problem convex and hence, efficiently solvable.

Our numerical results shows that the proposed two-stage algorithm has much less computational cost and offers higher performance gains in comparison with any 
currently available technique.

\subsection{List of Publication}

The following is a list of publications generated during the course of the Ph.D. Program.

\subsubsection{Published Journal Papers}

- Rozita Rashtchi, Ramy Gohary, and Halim Yanikomeroglu, "Generalized cross-layer designs for generic half-Duplex multicarrier wireless networks with frequency-reuse", accepted for publication in IEEE Transactions on Wireless Communications (acceptance date: 14 August 2015).

- Rozita Rashtchi, Ramy Gohary, and Halim Yanikomeroglu, "Routing, scheduling and power allocation in generic OFDMA wireless networks: Optimal design and efficiently computable bounds", IEEE Transactions on Wireless Communications, vol. 13, no. 4, pp. 2034-2046, April 2014.

\subsubsection{Published Conference Papers}

- Ramy Gohary, Rozita Rashtchi , and Halim Yanikomeroglu, "Optimal design and power allocation for multicarrier decode and forward relays", IEEE International Conference on Acoustics, Speech, and Signal Processing (ICASSP), 4-9 May 2015, Florence, Italy ${ }^{1}$.

- Rozita Rashtchi, Ramy Gohary, and Halim Yanikomeroglu, "A cross-layer design for generic half-duplex interference-limited multicarrier networks", 15th IEEE International Workshop on Signal Processing Advances in Wireless Communications (SPAWC), 22-25 June 2014, Toronto, Canada.

- Rozita Rashtchi, Ramy Gohary, and Halim Yanikomeroglu, "An efficient cross layer design for OFDMA-based wireless networks with channel reuse", IEEE Global Communications Conference (Globecom), 9-13 December 2013, Atlanta, GA, USA.

\footnotetext{
${ }^{1}$ This work was conducted during the fourth year of the $\mathrm{PhD}$ program. To maintain coherence and flow, this work is not included in this thesis.
} 
- Rozita Rashtchi, Ramy Gohary, and Halim Yanikomeroglu, "Efficiently computable bounds on the rates achieved by a cross layer design with binary scheduling in generic OFDMA wireless networks", IEEE Global Communications Conference (Globecom) Workshop: The 8th Broadband Wireless Access Workshop, 3-7 December 2012, Anaheim, California, USA.

- Rozita Rashtchi, Ramy Gohary, and Halim Yanikomeroglu, "Joint routing, scheduling and power allocation in OFDMA wireless ad hoc networks", IEEE International Conference on Communications (ICC), 10-15 June 2012, Ottawa, Canada.

\subsubsection{Journal Manuscripts}

- Rozita Rashtchi, Ramy Gohary, Halim Yanikomeroglu, Gamini Senarath and Ngoc-Dung Dao, "A Low-complexity algorithm for resource allocation in multihop OFDMA-based D2D communication Networks", awaiting clearance for submission to IEEE Transactions on Wireless Communications (patent application discussions with our industrial partner are under way).

\subsection{Organization of the Thesis}

The rest of the thesis is organized as follows. Chapter 2 provides a literature review of the problem considered herein. In Chapter 3, we explain the topology and design objective of the network under consideration. In Chapter 4, we consider the JRSPA problem when subcarriers are entitled to time-sharing but not to frequency-reuse. In Chapter 5, we consider the second case, when subcarriers are neither time-shared nor reused by multiple links. In Chapter 6, we consider the complementary case when, time-sharing of subcarriers is not allowed but the subcarriers can be reused by multiple links. The general case in which time-sharing and frequency-reuse are allowed is addressed in Chapter 7. In Chapter 8, we consider the general case for large-size networks and we propose a novel approach to solve it. A summary of this work, along with proposed future research directions are presented in Chapter 9. GP definitions, analyses pertaining thereto and equivalent self-concordant formulations are provided in the appendices. 


\section{Chapter 2}

\section{Literature Review}

\section{$2.1 \quad$ Introduction}

To put our work in perspective, in this chapter we provide a brief overview of the design techniques currently available for routing and resource allocation in wireless networks. A summary of this review is presented in Table 2.1. The entries of this table will be discussed next.

Efficient utilization of radio resources requires the use of optimization-based techniques for designing the network functionalities, including data routing, subcarrier scheduling and power allocation. While a plethora of techniques is available for optimizing each of these functionalities in isolation, significantly fewer techniques consider their optimization jointly.

Table 2.1: Related Work

\begin{tabular}{lcccccc}
\hline Platform & $\begin{array}{c}\text { Data } \\
\text { routing }\end{array}$ & $\begin{array}{c}\text { Power } \\
\text { allocation }\end{array}$ & $\begin{array}{c}\text { Subcarrier } \\
\text { scheduling }\end{array}$ & $\begin{array}{c}\text { Time } \\
\text { sharing }\end{array}$ & $\begin{array}{c}\text { Frequency } \\
\text { reuse }\end{array}$ & Reference \\
\hline Gaussian interference channel & $\times$ & $\checkmark$ & $\times$ & $\times$ & $\checkmark$ & {$[11-17]$} \\
\hline OFDMA cellular & $\times$ & $\checkmark$ & $\checkmark$ & $\times$ & $\times$ & {$[18-34]$} \\
\hline Relay network & $\checkmark$ & $\times$ & $\times$ & $\times$ & $\times$ & {$[35-38]$} \\
\hline OFDMA with 2-hop relay & $\checkmark$ & $\checkmark$ & $\checkmark$ & $\times$ & $\times$ & {$[39-47]$} \\
\hline OFDMA network & $\checkmark$ & $\checkmark$ & $\checkmark$ & $\checkmark$ & $\times$ & {$[48-50]$} \\
\hline
\end{tabular}




\subsection{Power Allocation in Single-carrier Networks}

Resource allocation in single-carrier wireless networks constitutes the task of determining the power allocated for the transmission of each node. Instances in which power allocation techniques were developed are provided in $[11,12,14-17]$ for various network scenarios. For instance, power allocation techniques are developed in [11] and [16] for systems with Gaussian interference channels. For high signal-to-noiseratio (SNR) regimes, the power allocation problem was cast in a GP form and the optimal power allocations were found in [16]. In contrast, for low SNR regimes, this problem is not a GP, but amenable to a technique called "monomial approximation". A general GP-based framework based on this technique was developed in [11] to obtain approximated power allocations. To obtain the optimal power allocations in this case, the authors in [12] used a technique called "polyblock outer approximation" which has high complexity. Several attempts have been made to develop low complexity algorithms for power allocation in systems with Gaussian interference channels. For instance, in [13] a small system with two users was considered and its optimal power allocation was found to be binary; each user either is silent or transmits with full power. For systems with more than two users, the authors in [14] proposed binary power allocation and they showed that if the level of interference in the system is less than a particular threshold, the optimal solution is to let all the users transmit at full power and if the level of interference is more than another threshold, the optimal solution is to let the user with the best channel condition transmit at full power and to let the other users remain silent. The optimal power allocation for all the interference levels has been confirmed to be binary in [15] through numerous numerical results. Recently in [17], the optimal power allocation has been analytically found for the uplink scenario of a single-cell cellular system and, using majorization theory, this allocation was shown to be binary.

\subsection{Joint Scheduling and Power Allocation in OFDMA Networks}

To enable more effective utilization of resources, power allocations were optimized jointly with binary-constrained subcarrier schedules, i.e., schedules that restricted each subcarrier to be used by only one node throughout the signalling interval and 
hence, the transmissions of the nodes do not interfere with each other.

The joint design of subcarrier schedules and power allocations was extensively considered in the literature for the downlink scenario of orthogonal frequency division multiplexing (OFDM) systems, see e.g., [18-23]. It was proved in [18] and [19] that the total throughput of a multiuser system is maximized if each subcarrier is assigned to the user with the best channel gain and the power is distributed using the water-filling policy. However, using this approach, the users with higher channel gains will be allocated most of the resources while leaving less for the users with low channel gains. To alleviate this difficulty, a related problem was considered in [21] with proportional fairness among the users, i.e., a set of proportional fairness constraints was imposed to assure that each user can achieve a required data rate. In this paper a low-complexity suboptimal algorithm that separates subcarrier scheduling and power allocation was proposed. In addition to fairness, in [22] the queue length of each user at the BS was also considered. Therein, a two-step algorithm was proposed to maximize the system throughput while maintaining a fairness among the users. Unlike the conventional water-filling algorithm, the practical consideration of discrete modulation levels was considered in [23] for the joint optimization of subcarrier schedules and power allocations. A survey on recent joint subcarrier scheduling and power allocation designs in downlink scenario of OFDM systems can be found in [7].

For the uplink scenario of wireless systems, the optimality of OFDMA was studied in [24] and it was shown that, while OFDMA is not optimal in general, the performance gap between the OFDMA scheme and the optimal solution is small in most cases. For this scenario an efficient algorithm based on the KKT conditions was developed in [25] and further investigated in [26] for scheduling the subcarriers and allocating power to them.

Further network performance improvements can be effected through relaxing the binary constraint on the subcarrier schedules and thereby allowing subcarriers to be time-shared by multiple links. For instance, when the receiver nodes experience self-noise, jointly optimal subcarrier scheduling and power allocation were obtained in [27] and [28] for the uplink and downlink scenarios of OFDM systems. For the downlink scenario, low complexity algorithms were developed in [29] and [30] to obtain the subcarrier schedules and power allocations sequentially. A related problem was considered in [31] and the design developed therein relied on the premise that the 
CSI is not perfect at the BS.

Efficient utilization of scarce radio resources suggests reusing the subcarriers in designing the network which, due to the resulting interference, makes the joint design problem more complicated. In this case, the power allocation problem is be NPhard [32]. To circumvent this difficulty, a heuristic algorithm was developed in [33] for the downlink scenario of OFDM systems. A related problem was considered in [34], wherein a successive convex approximation technique was used to obtain suboptimal solutions.

\subsection{Routing in Multihop Relay Networks}

To enhance coverage and performance of the future wireless networks, there has been increasing interest in integrating relaying functionalities into cellular systems [51]. There are two types of relay nodes in the network: 1) multihop relay to enhancing the network coverage [3]; 2) cooperative relay to increase the network performance [52]. In the latter case, a relay can employ different strategies for its transmission, e.g., Decode-and-Forward or Amplify-and-Forward [52]. Relays are also categorized based on their mobility, either fixed, nomadic or terminal relays [35]. In networks with multihop relays, routing traditionally has been done in isolation from the power allocation and subcarrier scheduling. For instance, the routing problem in networks with multihop relays was considered in [36] and in networks with cooperative relays was considered in $[37,38]$.

\subsection{JRSPA in Relay Networks}

Capitalizing on the advantages of considering multiple aspects jointly, several cross layer techniques have been developed for designing relay networks [53]. For instance, the joint design of data routes and power allocations was cast as a convex optimization problem for frequency-division-multiple-access (FDMA) systems in [54] and for code-division-multiple-access (CDMA) systems in [55]. To exploit the broadcast feature of the wireless medium, a locally optimal solution for data routes and power allocation is obtained in $[56,57]$. Therein, the nodes use superposition coding for transmission and the design is performed using the GP framework. An improvement on the design in [56] is proposed in [58] by allowing the nodes to reduce interference 
using a successive cancellation technique.

Another aspect to be considered jointly with subcarrier scheduling in relay-aided OFDMA cellular networks is the way in which data is routed across the network. In this case, communication between the BS and the users can be effected through two-hop routes and hence, designing the optimal routes is equivalent to selecting the best relay. In [39-43] several heuristic algorithms for the joint relay selection and subcarrier scheduling were developed for cases in which the relays operate in multihop fashion. The cases when relays operate in cooperative fashion were considered in [44-47] and, under various network constraints, optimal and suboptimal solutions were obtained.

\subsection{JRSPA in Generic OFDMA Networks}

Additional performance gains in OFDMA networks have been sought by incorporating more functionalities in the joint design of the network, i.e., joint data routing, subcarrier scheduling and power allocation. For instance, in $[48,49]$ heuristics were developed to optimize the data routes and subcarrier schedules jointly through LP. The power allocation in [48] was fixed and obtained a priori using the water-filling technique. The restriction of powers to be fixed was replaced with the one that allows powers to take discrete values in [50]. Therein, the joint design of routes, schedules and powers was cast in an LP form. The solution obtained therein can be considered as a lower bound on that corresponding to continuous power values.

In the current work, we consider the joint optimization of the power allocations, subcarrier schedules and data routes in the design of generic multicarrier networks with and without frequency-reuse, and with and without time-sharing. As such, the designs developed herein generalize currently available ones, and will subsequently offer a significant improvement over their performance. 


\section{Chapter 3}

\section{System Model and Preliminaries}

\subsection{Introduction}

In this chapter, we provide the network and communication models considered throughout this thesis.

\subsection{Network Model}

We consider a generic multicarrier wireless network with $N$ nodes and $L$ directed links. The presence of a link between two nodes indicates that the network is able to forward data from the start node to the end node of that link. The set of all destination nodes is denoted by $\mathcal{D}, \mathcal{D} \subseteq \mathcal{N}$. This network can be represented by a weighted directed graph with $N$ vertices, that is fully connected with $L=N(N-1)$ links. To facilitate enumeration of links, the link from node $n$ to node $n^{\prime}, n, n^{\prime} \in$ $\mathcal{N} \triangleq\{1, \cdots, N\}$, will be labelled by $\ell=(N-1)(n-1)+n^{\prime}-1$ if $n<n^{\prime}$ and by $\ell=(N-1)(n-1)+n^{\prime}$ if $n>n^{\prime}$. The set of all links is denoted by $\mathcal{L}$ and the sets of incoming and outgoing links of node $n \in \mathcal{N}$ are denoted by $\mathcal{L}_{-}(n)$ and $\mathcal{L}_{+}(n)$, respectively. The connectivity of this graph can be captured by an incidence matrix, $A=\left[a_{n \ell}\right]$, where $[56]$

$$
a_{n \ell}= \begin{cases}1 & \text { if link } \ell \in \mathcal{L}_{+}(n) \\ -1 & \text { if link } \ell \in \mathcal{L}_{-}(n) \\ 0 & \text { otherwise }\end{cases}
$$




\subsection{Communication Model}

In the considered multicarrier network, each node is assumed to have one transmit and one receive antenna, and a power budget, $P_{n}, n \in \mathcal{N}$. Nodes are assumed to be sources, destinations and/or relays. This assumption is generic, in the sense that constraining some nodes to perform a subset of these tasks can be readily incorporated in the forthcoming formulations. For tractability, the nodes will be assumed to always have data ready for transmission [54], and for practical considerations, the relaying nodes are assumed to operate in a half-duplex mode, whereby each node uses distinct physical channels for transmission and reception [48]. The relays operate in a multi-hop, rather than cooperative, fashion. We assume that the traffic flow can be split arbitrarily at nodes as long as the flow conservation law is satisfied at each node. It should be noted that this model describes the average behavior of data transmissions and ignores packet-level details of transmission protocols and forwarding mechanisms. The available frequency spectrum, $W_{0}$, is assumed to be divided into $K$ narrowband subcarriers, each of bandwidth $W=\frac{W_{0}}{K}$. The $K$ subcarriers are assumed to remain essentially constant during the entire signalling interval. In the considered multicarrier network, each link $\ell \in \mathcal{L}$ is composed of $K$ subcarriers, each with a complex random coefficient. The coefficient of the $k$-th subcarrier of link $\ell$ connecting node $n$ to node $n^{\prime}$ is denoted by $h_{n n^{\prime}}^{(k)}$.

In addition to the desired signals, the nodes receive a superposition of noise and interference, which is composed of the transmissions of all other nodes on the same subcarrier, scaled by the respective gains. Using $u_{n}^{(k)}$ and $y_{n}^{(k)}$ to denote the signals transmitted to and received by node $n$ on the $k$-th subcarrier, respectively, we can write

$$
y_{n^{\prime}}^{(k)}=\sum_{n \in \mathcal{N} \backslash\left\{n^{\prime}\right\}} h_{n n^{\prime}}^{(k)} u_{n}^{(k)}+v_{n^{\prime}}^{(k)}, \quad n^{\prime} \in \mathcal{N},
$$

where \denotes the set-minus operation and $v_{n^{\prime}}^{(k)}$ denotes the zero-mean additive Gaussian noise with variance of $N_{0}$.

In the current model, it is assumed that the nodes cannot broadcast data simultaneously on the same subcarrier to different destinations. However, because of superposition, this model resembles a multiple access channel, wherein the nodes might be able to jointly decode the signals of other nodes. Such decoding involves successive detection and cancellation in a certain order, which makes the network design rather complicated. A more pragmatic approach is for each node to decode 
the signal received on each link separately, while treating the signals received on other links as additive interference. In this case, assuming, as before, that $\ell \in \mathcal{L}$ is the link connecting node $n$ to node $n^{\prime}$, it can be seen from (3.2) that the signal-tointerference-plus-noise ratio (SINR) observed by node $n^{\prime}$ on subcarrier $k$ of link $\ell$ is given by

$$
\operatorname{SINR}(\ell, k)=\frac{p_{n}^{(k)}\left|h_{n n^{\prime}}^{(k)}\right|^{2}}{W N_{0}+\sum_{n^{\prime \prime} \in \mathcal{N} \backslash\left\{n, n^{\prime}\right\}} p_{n^{\prime \prime}}^{(k)}\left|h_{n^{\prime \prime} n^{\prime}}^{(k)}\right|^{2}}, \quad \ell \in \mathcal{L}, k \in \mathcal{K},
$$

where $p_{n}^{(k)}$ is the power allocated by node $n$ to the $k$-th subcarrier and $N_{0}$ is the spectral density of the additive white Gaussian noise at destination nodes. The second term in the denominator of (3.3) represents the aggregate interference observed by node $n^{\prime}$ on subcarrier $k$ of link $\ell$. When the nodes transmit Gaussian distributed signals, the maximum data rate that can be reliably communicated on this subcarrier is given by $W \log _{2}(1+\operatorname{SINR}(\ell, k))$.

For notational convenience, we will use the fact that the index of each link $\ell$ corresponds to a specific $\left(n, n^{\prime}\right)$ pair and will use $g_{\ell k}$ to denote the normalized channel gain, $\frac{\left|h_{n n^{\prime}}^{(k)}\right|^{2}}{W N_{0}}$, for any two nodes $n, n^{\prime} \in \mathcal{N}$.

\subsection{Generic Network}

The nodes of the network considered in this thesis can assume multiple roles simultaneously including being sources, destinations and/or relays; constraining some nodes to perform a subset of these tasks can be readily incorporated in the forthcoming formulations. This assumption is generic in the sense that it encompasses many existing and upcoming network structures as special cases. In this section we will provide few examples of networks which can be considered as special cases of the generic network considered in this paper.

Cellular Networks: In cellular networks each cell is served by at least one fixedlocation BS. UTs are connected to the nearest BS with star topology. Each user is served by only one BS. As all the BSs share common pool of resources, resource management is critical in such networks. Figure 3.1 illustrates an example of such networks. In this figure, in the downlink (uplink) scenario, BSs act as sources (destinations) and UTs act as destinations (sources). Note that, 
as UTs are directly connected to BSs, there is no relaying node and hence, no multihop routing.

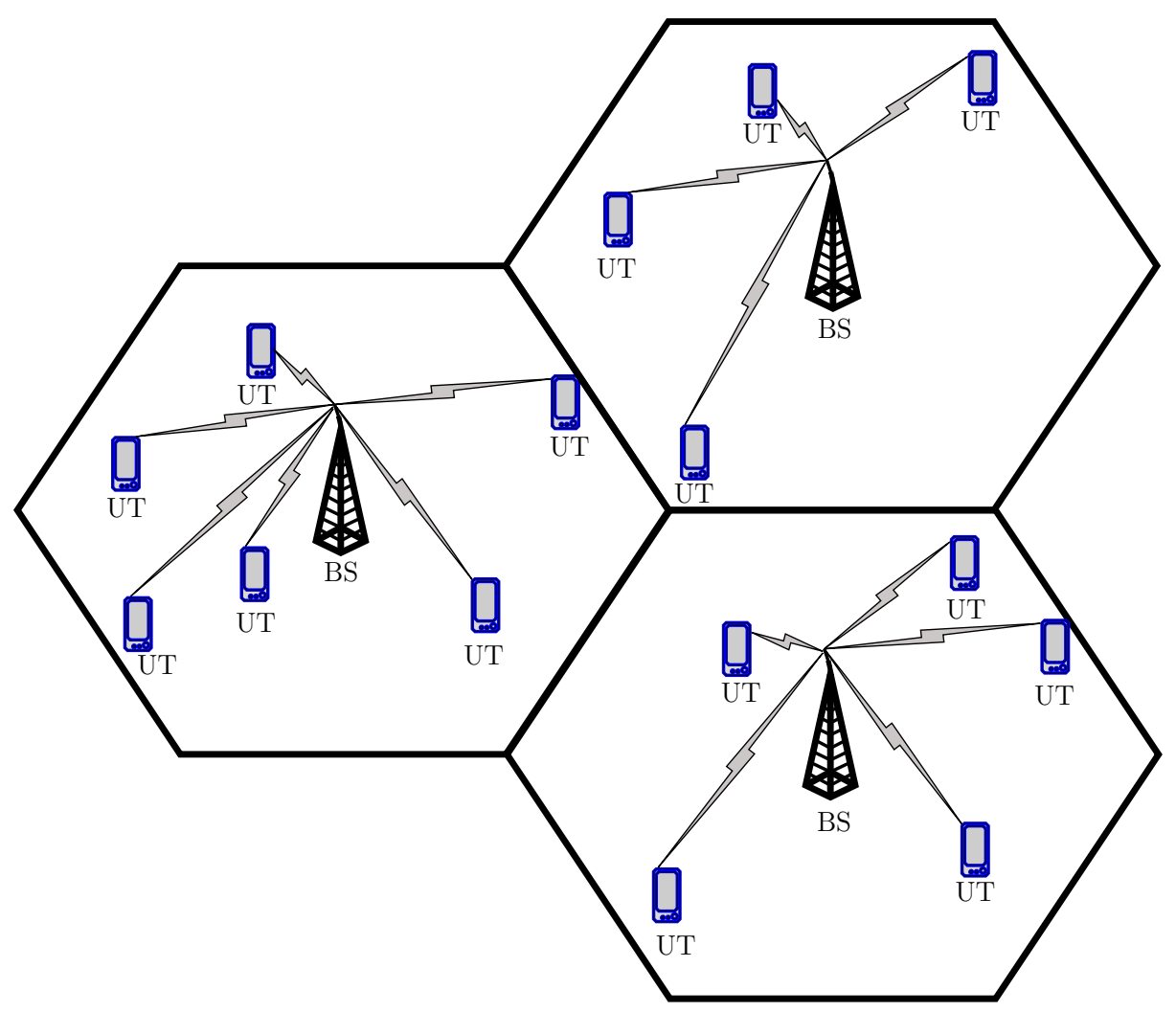

Figure 3.1: A cellular network.

Relay Assisted Cellular Networks: To have ubiquitous coverage, it is advantageous for network service providers to distribute system capacity across the network area, reaching UTs in the most cost-effective way. With the traditional cellular architecture, increasing capacity and improving coverage requires the deployment of a large number of BSs. This approach is cost prohibitive to network service providers. As an alternative, relaying techniques are expected to alleviate this coverage problem since a relay station is usually less capable than a BS, but can forward high data rates to remote areas of the cell while lowering infrastructure cost. Fixed relaying which involves the deployment of low-power BSs to assist cellular communications has been extensively studied 
in the literature, e.g., $[3,35,42]$ and it has already been included in the fourthgeneration $(4 \mathrm{G})$ long-term evolution (LTE)-Advanced standard. Figure 3.2 illustrates an example of such networks. In this figure, in the downlink (uplink) scenario, BSs act as sources (destinations), UTs act as destinations (sources) and relay stations assist communication between sources and destinations.

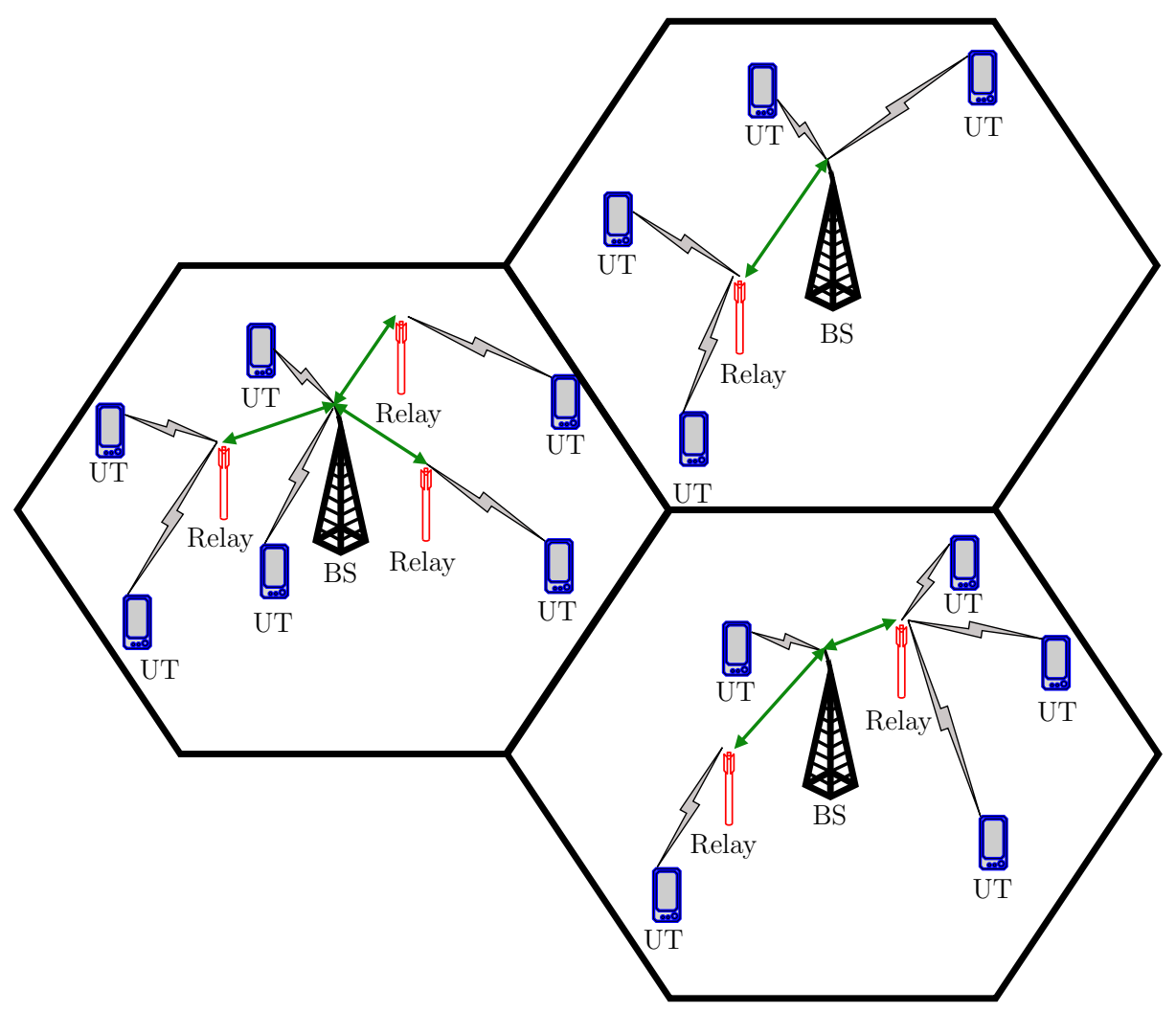

Figure 3.2: A relay-assisted cellular network.

Mesh Backhaul Networks: A mesh network is an efficient backhaul solution when the cost of connecting BSs or access points by a wired backhaul is too high, mainly because of the cable deployment cost [48]. This is specially true in wireless networks with smaller cell sizes. In this case it is important to provide a cost-effective backhaul solution for a large number of access points serving a given coverage area. Moreover, an efficient backhaul may provide extended network coverage through multi-hop routing and is also necessary for cooperative interference mitigation techniques. Figure 3.3 illustrates an example of such networks. In this figure, in the downlink (uplink) scenario, the wireless 
gateways which are connected to Internet serve as sources (destinations), UTs and potentially BSs serve as destinations (sources), and BSs serve as relays.

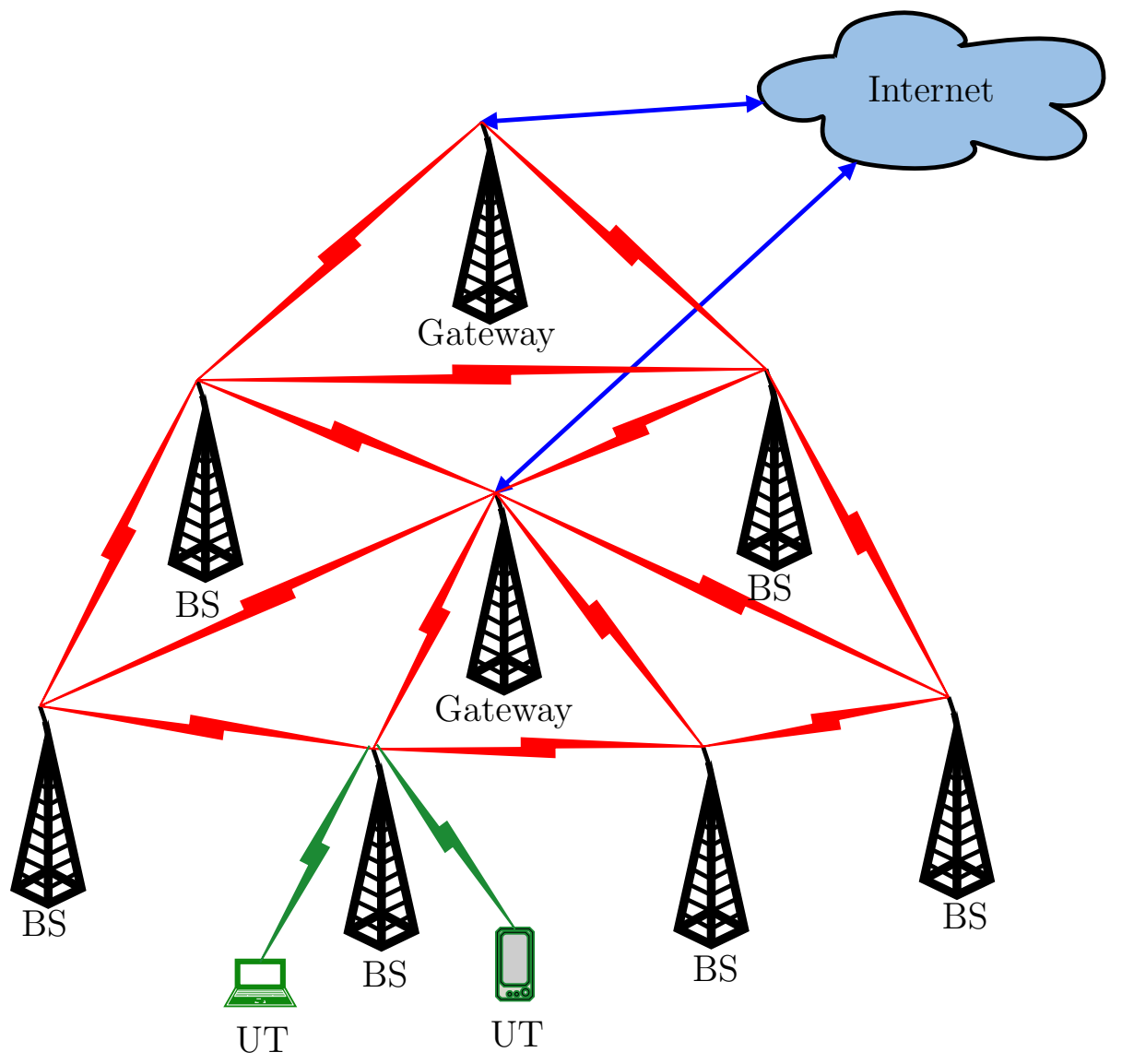

Figure 3.3: A mesh backhaul network.

D2D Communications Networks: With the rapid growth of context-aware and location-aware services such as social networks, D2D communications within cellular network has attracted enormous research interest [59]. In D2D communications, two devices are allowed to communicate in the licensed cellular bandwidth with or without BS involvement [5]. One of the most challenging research topics is how to develop high-performance techniques for routing and radio resource management in D2D networks. This challenge arises from the fact that the number of devices with higher demands increases in cellular networks and hence low-complexity algorithms are required to design such networks. Figure 3.4 illustrate an example of such networks. In this figure, 
different data streams are identified by numbers. As can be seen in this figure, while some UTs are acting only as sources, destinations or relays, others perform multiple tasks simultaneously.

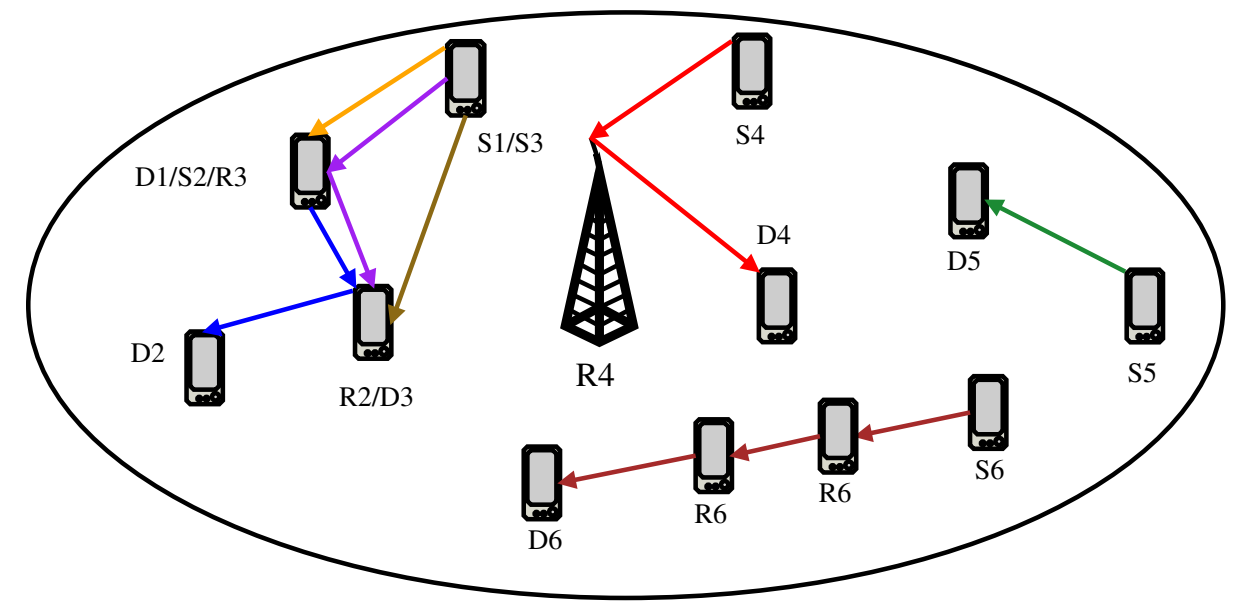

Figure 3.4: A D2D communication network.

\subsection{Design Variables}

\subsubsection{Routing Variables}

Characterizing the data routes between various source-destination pairs can be effected through the data flows on all subcarriers of each link. The flows are distinguished by the intended destination. In particular, we denote $x_{\ell k}^{(d)}$ to be the data flow intended for destination $d \in \mathcal{D}$ on subcarrier $k \in \mathcal{K}$ of link $\ell \in \mathcal{L}$. Also, we denote $s_{n}^{(d)}$ to be the rate of the data stream injected into node $n \in \mathcal{N}$ and intended for destination $d \in \mathcal{D}$. An instance of a network considered herein with $N=4$ nodes, $L=12$ links and $K=2$ subcarriers (solid and dashed lines) is depicted in Figure 3.5. In this figure, nodes 1 and 2 are destination nodes, $D=\{1,2\}$.

\subsubsection{Power Allocation Variables}

In the considered network, the relaying nodes operate in the half-duplex mode and the nodes cannot simultaneously broadcast to multiple destinations on the same 


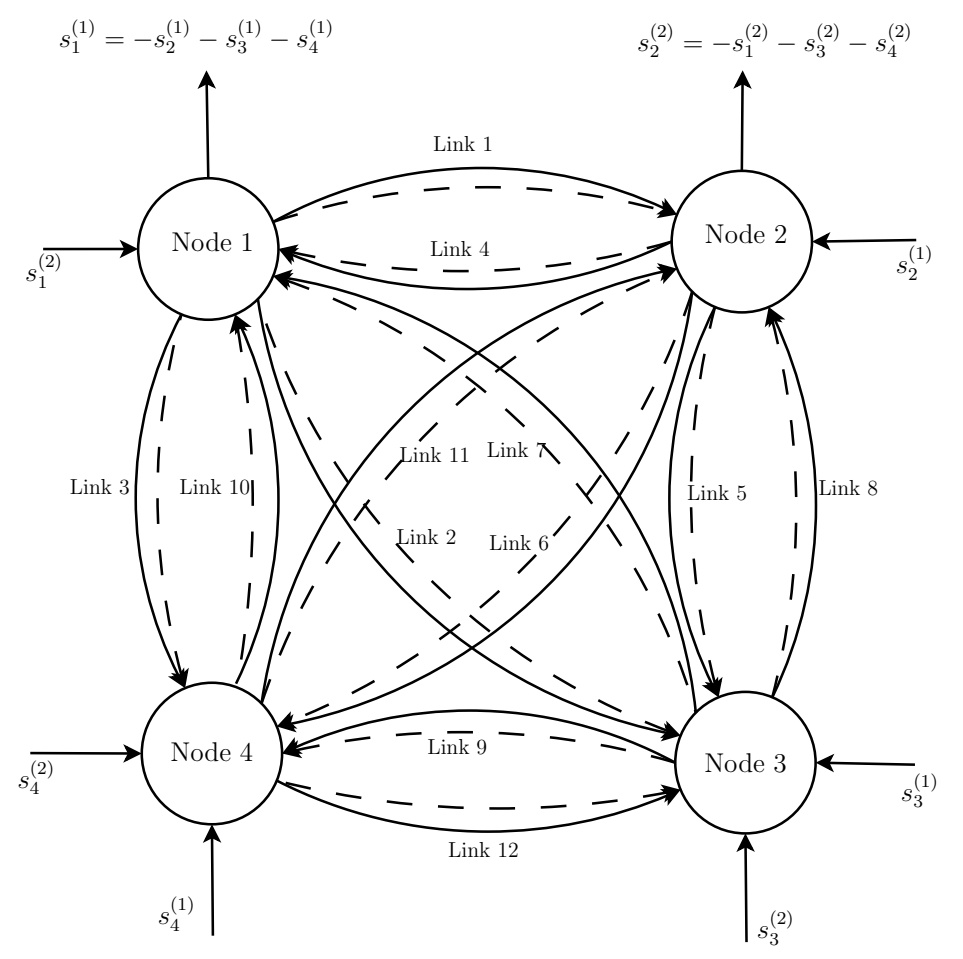

Figure 3.5: A network with $N=4, K=2$ (solid and dashed lines) and $D=\{1,2\}$.

subcarrier. To capture these requirements, we distinguish between the transmissions on incoming and outgoing links of node $n \in \mathcal{N}$. In particular, we introduce the variables $\left\{q_{\ell k}\right\}, \ell \in \mathcal{L}, k \in \mathcal{K}$ which refer to the link powers. These variables are related to the node powers by the following set of transformations:

$$
\begin{aligned}
& a_{n \ell}^{+} a_{n \ell^{\prime}}^{+} q_{\ell k} q_{\ell^{\prime} k}=0, \quad \ell, \ell^{\prime} \in \mathcal{L}, k \in \mathcal{K}, n \in \mathcal{N}, \\
& p_{n}^{(k)}=\max _{\ell \in \mathcal{L}_{+}(n)} q_{\ell k}, \quad n \in \mathcal{N}, k \in \mathcal{K},
\end{aligned}
$$

where $a_{n \ell}^{+}=\max \left\{0, a_{n \ell}\right\}$, that is, $a_{n \ell}^{+}=1$ if $\ell \in \mathcal{L}_{+}(n)$ and zero otherwise.

To gain a better understanding of the transformation in (3.4), we note that (3.4a) implies that for any subcarrier $k \in \mathcal{K}$ and any two links $\ell, \ell^{\prime} \in \mathcal{L}_{+}(n)$, at least $q_{\ell k}=0$ or $q_{\ell^{\prime} k}=0$. In other words, the equality in (3.4a) implies that, of all the links in $\mathcal{L}_{+}(n)$, only one element in the set $\left\{q_{\ell k}\right\}_{\ell \in \mathcal{L}_{+}(n)}, \forall n \in \mathcal{N}, k \in \mathcal{K}$, can assume a strictly positive value. Now, (3.4b) indicates that this value is the power allocated by node $n$ to subcarrier $k$. Note that using (3.4) will enable us to formulate the design in terms of $\left\{q_{\ell k}\right\}$ instead of $\left\{p_{n}^{(k)}\right\}$. 


\subsubsection{Scheduling Variables}

To characterize the constraints that must be satisfied by subcarrier schedules, let $c_{\ell k}$ be a variable that determines the fraction of time during which link $\ell \in \mathcal{L}$ is scheduled to use subcarrier $k \in \mathcal{K}$.

The four categories of design problems discussed in Section 1.2 can be represented in terms of scheduling variables, $\left\{c_{\ell k}\right\}$. Scheduling samples of these four categories are shown in Figure 3.6 for a network with $L=3$ links and $K=1$ subcarrier.

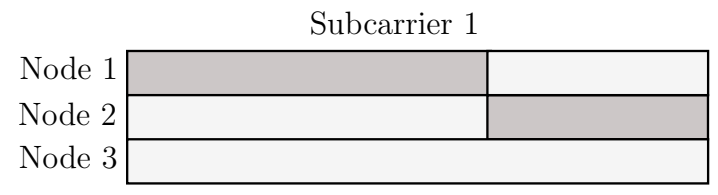

(a)

Subcarrier 1

\begin{tabular}{l|l|} 
Node 1 & \\
Node 2 & \\
\cline { 3 - 3 } Node 3 &
\end{tabular}

(c)

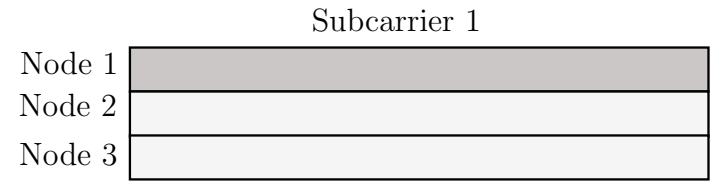

(b)

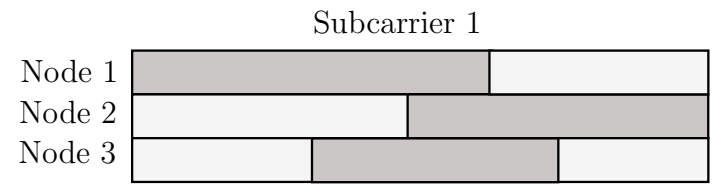

(d)

Figure 3.6: Scheduling samples for designs (a) w. time-sharing, w/o frequency-reuse, (b) w/o time-sharing, w/o frequency-reuse, (c) w/o time-sharing, w. frequencyreuse, and (d) w. time-sharing, w. frequency-reuse. Dark sections represent transmission intervals and light sections represents silent intervals.

\subsection{Design Objective}

Let $w_{n}^{(d)}$ be the non-negative weight assigned to $s_{n}^{(d)}$. Our objective is to maximize the weighted-sum of the rates injected into the network, i.e., $\max \sum_{d \in \mathcal{D}} \sum_{n \in \mathcal{N}, n \neq d} w_{n}^{(d)} s_{n}^{(d)}$, for some given $\left\{w_{n}^{(d)}\right\}$ satisfying $\frac{1}{D(N-1)} \sum_{d \in \mathcal{D}} \sum_{n \in \mathcal{N} \backslash\{d\}} w_{n}^{(d)}=1$. A particular weight assignment is when all rates are assigned the same weight, i.e., $w_{n}^{(d)}=1$, for all $n \in \mathcal{N}$ and $d \in \mathcal{D}$.

From a practical perspective, assigning weights to injected rates provides a convenient means for controlling the quality of service (QoS) offered to various network users; a higher weight implies a higher priority to the corresponding rate. Such weights are typically assigned a priori, but can be adapted to meet variations in the QoS requirements [28]. Another advantage of considering weighted-sum rates is that 
varying the weights over the unit simplex enables us to evaluate the region of all injected rates that, by using a specific design, can be communicated over the network during a signalling interval. To see the utility of assigning weights to rates rather than users, we note that since there are multiple sources, a user may wish to assign different priorities to different sources. Hence, assigning weights to rates is more general than assigning them to users.

\subsection{Implementation}

We now provide a brief discussion on the implementation of the joint design. To begin with, we note that the design considered in this thesis is centralized, in the sense that the design is performed by a central entity that is aware of the network parameters. The signalling exchange between the nodes and the central entity, required to establish communication in the considered framework, are described as follows. At the beginning of each signalling interval, the central entity prompts the nodes in the network to sequentially broadcast pilot signals of prescribed power levels. Subsequently, each node computes the subcarrier channel gains from all other nodes in the network. There is total of $L K$ such gains, where $L$ is the number of links and $K$ is the number of subcarriers. Each node sends these gains along with its destination nodes, if any, and its priority weights to the central entity. The central entity performs the joint optimization of the power allocations, scheduling parameters and data routes. It then forwards these decisions to all the nodes, possibly over a dedicated control channel. In particular, the information forwarded by the central entity include

1. The subcarrier index allocated to each transmission. This information is provided by the set $\left\{c_{\ell k}\right\}$ and the cardinality of this set is $L K$;

2. The power allocated to each transmission. This information is provided by the set $\left\{q_{\ell k}\right\}$ and the cardinality of this set is $L K$; and

3. The data rates at each transmitting and receiving node in the route of the stream intended for each destination. This information is provided in the set $\left\{x_{\ell k}^{(d)}\right\}$ and the cardinality of this set is $L K D$, where $D$ is the number of intended destinations.

We note that the joint design problem must be re-solved at each scheduling instance because of changes in the channel and weights. While the former change is 
due to the time-varying nature of wireless channels, the latter change is due to new arrivals and past service decisions. We also note that the designs developed in this thesis update the variables at the same time. This is in contrast to the conventional communication systems where the time-scale at which the routes are updated is larger than the time-scale at which the schedules and powers are updated. 


\section{Chapter 4}

\section{JRSPA with Time-Sharing and without Frequency-Reuse}

\subsection{Introduction}

In this chapter, we consider the JRSPA problem in a generic multicarrier network in which each subcarrier is scheduled to be used exclusively by one node at any given time instant, refereed to as "continuous scheduling". As such, each subcarrier can be time-shared among multiple links, but cannot be reused over the network.

\subsection{System Constraints}

To ensure realizability of the prospective design, data routes must satisfy network layer constraints, whereas subcarrier schedules and power allocations must satisfy medium access control (MAC) layer constraints. The constraints from both network and MAC layers are coupled by the capacity of each wireless link, which imposes a physical layer constraint. These constraints, their implications, and their interdependence will be elucidated in this section.

\subsubsection{Routing Constraints}

To characterize the routing constraints, we note that the flows, $\left\{x_{\ell k}^{(d)}\right\}$, and the injected rates, $\left\{s_{n}^{(d)}\right\}$, are related by the flow conservation law, which must be satisfied at each node in the network. This law stipulates that the sum of all flows intended for each destination $d \in \mathcal{D}$ at each node must be equal to zero [54]. This guarantees the 
existence of continuous routes between sources and destinations. Hence, to obtain the routes that enable the maximum weighted-sum rate to be achieved, we will include $\left\{x_{\ell k}^{(d)}\right\}$ in the cross-layer design framework. Using the incidence matrix in (3.1) to distinguish between incoming and outgoing links, it can be seen that $\left\{x_{\ell k}^{(d)}\right\}$ and $\left\{s_{n}^{(d)}\right\}$ must satisfy the following constraints:

$$
\sum_{\ell \in \mathcal{L}} \sum_{k \in \mathcal{K}} a_{n l} x_{\ell k}^{(d)}=s_{n}^{(d)}, \quad \forall n \in \mathcal{N}, \forall d \in \mathcal{D}, n \neq d .
$$

Successive application of the flow conservation law yields that the total data rate received by any destination node $d \in \mathcal{D}$ is given by $s_{d}^{(d)}=-\sum_{n \in \mathcal{N} \backslash\{d\}} s_{n}^{(d)}$. The fact that $s_{d}^{(d)}$ is a negative quantity implies that this rate "leaves" the network.

The injected rates, $\left\{s_{n}^{(d)}\right\}_{n \neq d}$, are non-negative and since in our model the network is represented by a directed graph, the flows, $\left\{x_{\ell k}^{(d)}\right\}$, must be also non-negative. Hence,

$$
\begin{array}{ll}
x_{\ell k}^{(d)} \geq 0, & \forall \ell \in \mathcal{L}, \forall k \in \mathcal{K}, \forall d \in \mathcal{D}, \\
s_{n}^{(d)} \geq 0, & \forall n \in \mathcal{N} \quad \forall d \in \mathcal{D}, n \neq d .
\end{array}
$$

For mathematical tractability, the source nodes will be assumed to have an infinite backlog, and thus they always have data ready for transmission [60].

\subsubsection{Scheduling Constraints}

When time-sharing is allowed, $\left\{c_{\ell k}\right\}$ assume continuous values, i.e.,

$$
c_{\ell k} \in[0,1], \quad \forall \ell \in \mathcal{L}, \forall k \in \mathcal{K},
$$

In the considered multicarrier structure, interference is avoided by restricting each subcarrier to be used at most once across the entire network [44]. Hence, with (4.4) satisfied, this requirement can be expressed as

$$
\sum_{\ell \in \mathcal{L}} c_{\ell k} \leq 1, \quad \forall k \in \mathcal{K}
$$




\subsubsection{Power Allocation Constraints}

To determine the constraints that must be satisfied by any feasible power allocation, first we note that the elements of $\left\{q_{\ell k}\right\}$ must satisfy

$$
q_{\ell k} \geq 0, \quad \forall \ell \in \mathcal{L}, \forall k \in \mathcal{K} .
$$

In a practical network, each node $n \in \mathcal{N}$ has a energy budget, $P_{n}$, which bounds the total power allocated by each node on outgoing links and subcarriers. This constraint can be written as

$$
\sum_{k \in \mathcal{K}} \sum_{\ell \in O(n)} c_{\ell k} q_{\ell k} \leq P_{n}, \quad \forall n \in \mathcal{N}
$$

In writing (4.7), we have used the fact that only the subcarriers scheduled to outgoing links contribute to the power consumed by every node.

\subsubsection{Capacity Constraints}

The data routes, subcarrier schedules and power allocations are coupled by the maximum rate that can be reliably communicated on the subcarriers of each link, i.e., subcarrier capacities. In other words, the aggregate flow on each subcarrier $k \in \mathcal{K}$, of each link $\ell \in \mathcal{L}, \sum_{d=1}^{D} x_{\ell k}^{(d)}$, must not exceed the capacity of this subcarrier. Assuming that the nodes use Gaussian signaling, the capacity of subcarrier $k \in \mathcal{K}$ of link $\ell \in \mathcal{L}$ can be expressed as $W \log _{2}\left(1+q_{\ell k} g_{\ell k}\right)$. When the $k$-th subcarrier is used on the $\ell$-th link for a fraction $c_{\ell k}$ of the signalling interval, the aggregate flow on this subcarrier must not exceed $W c_{\ell k} \log _{2}\left(1+q_{\ell k} g_{\ell k}\right)$, that is,

$$
\sum_{d \in \mathcal{D}} x_{\ell k}^{(d)} \leq W c_{\ell k} \log _{2}\left(1+q_{\ell k} g_{\ell k}\right), \forall \ell \in \mathcal{L}, \forall k \in \mathcal{K}
$$

The following remark exposes the generality of the considered model.

Remark 1 (Special Configurations).

- Prohibiting a node from being a source can be accomplished by setting its injected rate to zero, and prohibiting a node from being a destination can be accomplished by excluding its index from $\mathcal{D}$. 
- Restricting a node to be a source can be accomplished by setting all the subcarrier gains of incoming links to zero. Likewise, restricting a node to be a destination can be accomplished by setting all the subcarrier gains of outgoing links to zero.

\subsection{Problem Formulation}

Combining the objective in Section 3.6 with the constraints in (4.1)-(4.8), the JRSPA design problem can be cast as the following optimization problem:

$$
\max _{\left\{s_{n}^{(d)}\right\},\left\{x_{\ell k}^{(d)}\right\},\left\{c_{\ell k}\right\},\left\{q_{\ell k}\right\}} \sum_{d} \sum_{n, n \neq d} w_{n}^{(d)} s_{n}^{(d)}
$$

subject to

$$
\begin{array}{lr}
\sum_{\ell \in \mathcal{L}} \sum_{k \in \mathcal{K}} a_{n \ell} x_{\ell k}^{(d)}=s_{n}^{(d)}, & \forall n \in \mathcal{N}, \forall d \in \mathcal{D}, n \neq d, \\
x_{\ell k}^{(d)} \geq 0, & \forall \ell \in \mathcal{L}, \forall k \in \mathcal{K}, \forall d \in \mathcal{D}, \\
s_{n}^{(d)} \geq 0, & \forall n \in \mathcal{N}, \forall d \in \mathcal{D}, n \neq d, \\
\sum_{d \in \mathcal{D}} x_{\ell k}^{(d)} \leq W c_{\ell k} \log _{2}\left(1+q_{\ell k} g_{\ell k}\right), & \forall \ell \in \mathcal{L}, \forall k \in \mathcal{K}, \\
c_{\ell k} \in[0,1], & \forall \ell \in \mathcal{L}, \forall k \in \mathcal{K}, \\
q_{\ell k} \geq 0, & \forall \ell \in \mathcal{L}, \forall k \in \mathcal{K}, \\
\sum_{\ell \in \mathcal{L}} c_{\ell k} \leq 1, & \forall k \in \mathcal{K}, \\
\sum_{k \in \mathcal{K}} \sum_{\ell \in O(n)} c_{\ell k} q_{\ell k} \leq P_{n}, & \forall n \in \mathcal{N} .
\end{array}
$$

The objective and the constraints in this formulation are linear, except the constraints in $(4.9 \mathrm{e})$ and (4.9i). For the constraint in (4.9e), the left hand side (LHS) is linear. However, as can be readily verified by direct computation of the Hessian matrix, the right hand side (RHS) of $(4.9 \mathrm{e})$ is not jointly concave in $\left(c_{\ell k}, q_{\ell k}\right)$. For the constraint in (4.9i), the RHS is linear, but the LHS is not jointly convex in $c_{\ell k}$ and $q_{\ell k}$, as can be verified by direct computation of the Hessian matrix. 


\subsection{The Optimal Solution}

The nonconvexity of (4.9e) and (4.9i) renders the JRSPA design in (4.9) difficult to solve. This difficulty can be circumvented by using the following change of variables. Let

$$
y_{\ell k}=c_{\ell k} q_{\ell k}, \quad \forall \ell \in \mathcal{L}, \forall k \in \mathcal{K} .
$$

Since $\left\{c_{\ell k}\right\}$ are nonnegative, it can be seen that this change of variables yields a oneto-one mapping from $\left\{y_{\ell k}\right\}$ to $\left\{q_{\ell k}\right\}$; when $c_{\ell k}=0, q_{\ell k}$ can be set to zero without loss of optimality. A similar change of variables was used in [29] in a different optimization framework. Using $\left\{y_{\ell k}\right\}$ instead of $\left\{q_{\ell k}\right\}$, the RHS of $(4.9 \mathrm{e})$ can be expressed as $W c_{\ell k} \log _{2}\left(1+\frac{y_{\ell k} g_{\ell k}}{c_{\ell k}}\right)$.

We now recall the following result from [8]. Let $g(u)$ be a function and $f(t, u)$ be its perspective: $f(t, u) \triangleq \operatorname{tg}\left(\frac{u}{t}\right)$. Then $g(u)$ is concave in $u$ if and only if $f(t, u)$ is concave in $(t, u)$. Now, $W c_{\ell k} \log _{2}\left(1+\frac{y_{\ell k} g_{\ell k}}{c_{\ell k}}\right)$ is the perspective of $W \log _{2}\left(1+y_{\ell k} g_{\ell k}\right)$. Since this function is concave in $y_{\ell k}$, we conclude that $W c_{\ell k} \log _{2}\left(1+\frac{y_{\ell k} g_{\ell k}}{c_{\ell k}}\right)$ is concave in $\left(c_{\ell k}, y_{\ell k}\right)$.

Using this observation and the change of variables in (4.10), the JRSPA problem can be cast in the following convex form:

$$
\max _{\left\{s_{n}^{(d)}\right\},\left\{x_{\ell k}^{(d)}\right\},\left\{c_{\ell k}\right\},\left\{y_{\ell k}\right\}} \sum_{d} \sum_{n, n \neq d} w_{n}^{(d)} s_{n}^{(d)}
$$

subject to

$$
\begin{array}{lr}
\sum_{\ell \in \mathcal{L}} \sum_{k \in \mathcal{K}} a_{n \ell} x_{\ell k}^{(d)}=s_{n}^{(d)}, & \forall n \in \mathcal{N}, \forall d \in \mathcal{D}, n \neq d, \\
x_{\ell k}^{(d)} \geq 0, & \forall \ell \in \mathcal{L}, \forall k \in \mathcal{K}, \forall d \in \mathcal{D}, \\
s_{n}^{(d)} \geq 0, & \forall n \in \mathcal{N}, \forall d \in \mathcal{D}, n \neq d, \\
\sum_{d \in \mathcal{D}} x_{\ell k}^{(d)} \leq W c_{\ell k} \log _{2}\left(1+\frac{y_{\ell k} g_{\ell k}}{c_{\ell k}}\right), & \forall \ell \in \mathcal{L}, \forall k \in \mathcal{K}, \\
c_{\ell k} \geq 0, y_{\ell k} \geq 0, & \forall \ell \in \mathcal{L}, \forall k \in \mathcal{K}, \\
\sum_{\ell \in \mathcal{L}} c_{\ell k} \leq 1, & \forall k \in \mathcal{K}, \\
\sum_{k \in \mathcal{K}} \sum_{\ell \in \mathcal{L}_{+}(n)} y_{\ell k} \leq P_{n}, & \forall n \in \mathcal{N} . \\
\end{array}
$$

From this formulation it can be seen that the objective is linear and all, but (4.11e), 
are linear constraints. However, we have shown that (4.11e) is also convex. Hence, we conclude that (4.11) is a convex optimization problem which can be solved efficiently. Being strictly feasible, it can be seen that the global optimal solution of this problem can be found in polynomial time [8].

\subsection{Complexity Analysis}

In this section we will examine the computational complexity required for solving the problem in (4.11).

The formulation for the cross-layer design provided in Section 4.4 is convex and hence highly-efficient IPM-based solvers can be utilized to attain its global optimal. The philosophy that underlies IPMs is to use the objective and the inequality constraints to synthesize a log-barrier function, which is then minimized using Newton's method along a central path. The complexity of each Newton step along this path can be shown to grow with the cube of the number of constraints, and the number of Newton steps can be bounded, if the log-barrier function possesses the so-called self-concordance property [61], cf. Appendix C.1.

Unfortunately, verifying the self-concordance property for the log-barrier function corresponding to the formulation in (4.11) is overly complicated. Hence, to assess the complexity of solving it, in Appendix C.2 we introduce auxiliary constraints that do not affect the solution, but enables us to cast the log-barrier function in a selfconcordant form. Using this approach we arrive at the following result.

Proposition 1. The complexity of solving (4.11) is of order

$$
\mathcal{O}\left((L K(4+D)+N+K+D(N-1))^{3.5}\right)
$$

Proof. See Appendix C.2.

Proposition 1 shows that the computational complexity required for finding the optimal JRSPA design with time-sharing is polynomial in the number of nodes, $N$, and subcarriers, $K$. Hence, the complexities of the optimal design is relatively small. In particular, the computational complexity of the optimal JRSPA with time-sharing grows as $K^{3.5} N^{7}$. 


\subsection{Numerical Results}

In this section we provide numerical examples to evaluate the performance of the cross-layer design approach presented in Sections 4.4 for the continuous scheduling framework without frequency-reuses. We present two examples. In the first example, we assess the performance of the optimal JRSPA design with time-sharing and we obtain the region of rates achievable by this design technique. In the second example, we apply the design technique of Section 4.4 in a 10-node LTE-based network. We illustrate the sum rate achieved by this technique and compare it with the fixed power allocation counterpart.

For all examples, the mathematical programs are solved using the CVX package [62] with underlying Sedumi [63] and MOSEK [64] solvers.

Example 1: (Performance Evaluation) In this section we provide a numerical example of a network with $N=3$ nodes, $L=6$ links, and $K=4$ subcarriers of unit bandwidth each, i.e., $W=1$. This network is depicted in Figure 4.1. We consider

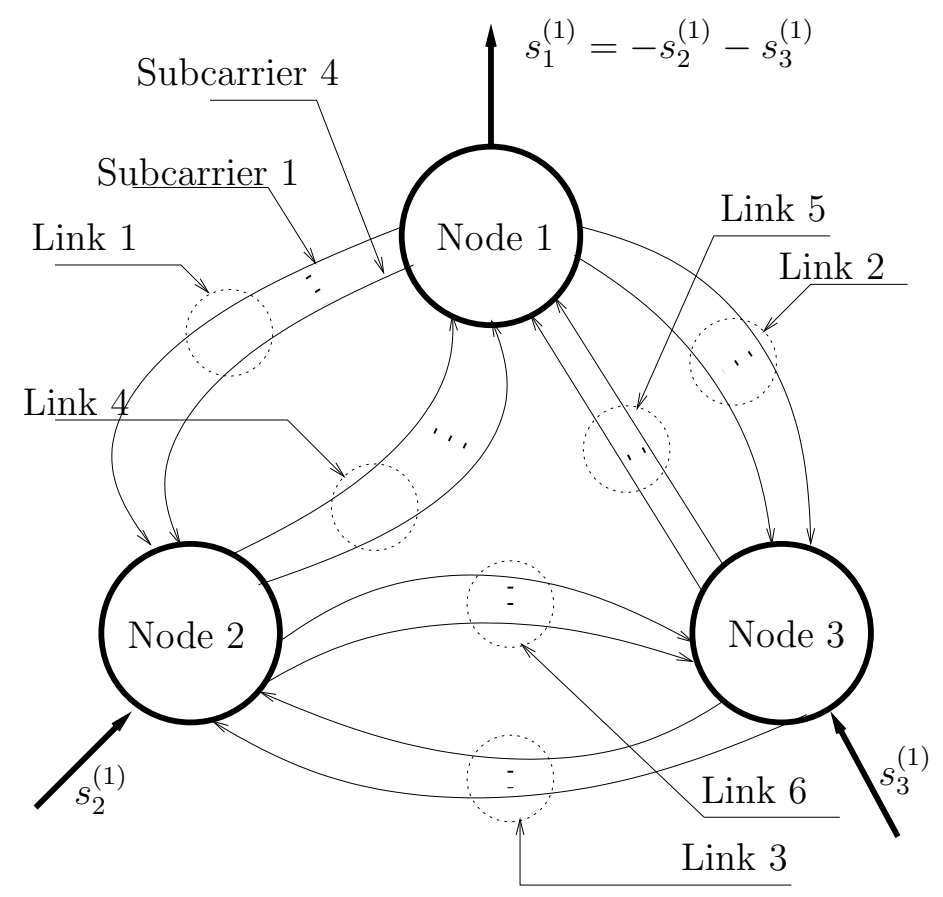

Figure 4.1: Network schematic with $N=3, L=6, K=4$ and $D=1$.

a quasi stationary setup with a fixed realization of the channel gains, $\left\{g_{\ell k}\right\}$, drawn from the standard circularly symmetric Gaussian distribution. The values of these 
gains, $g_{\ell k}$, are given in the following matrix:

$$
\left[g_{\ell k}\right]=\left[\begin{array}{cccc}
0.3803 & 0.3576 & 0.1657 & 0.1260 \\
0.0369 & 0.3098 & 0.4149 & 0.0389 \\
0.3169 & 0.7361 & 0.1134 & 0.1988 \\
0.2057 & 0.3733 & 0.4316 & 0.0453 \\
0.2589 & 0.3697 & 0.0781 & 0.3457 \\
0.3441 & 0.1846 & 0.2002 & 0.0630
\end{array}\right]
$$

First, we consider the case of maximizing the sum of the rates that nodes 2 and 3 can communicate to node 1 . In this case, $w_{1}^{(d)}=0, s_{1}^{d}=0, d=2,3, w_{n}^{(1)}=0.5$, $s_{n}^{(1)} \geq 0, n=2,3$. The number of optimization variables corresponding to this setup is 74 and the number of constraints is 107 .

When $P_{n}=20 \mathrm{~dB}, n=1,2,3$, the sum rate achieved by solving (4.11) directly (with the continuous scheduling constraints) is 16.02 bits per seconds per hertz (bits/s/Hz).

It is interesting to look at the scheduling variables obtained by solving (4.11). Solving (4.11) yields $c_{3 k}=1, k=2,3, c_{54}=1, c_{31}=0.25, c_{51}=0.75$, and $c_{\ell k}=0$ otherwise. This implies that the optimal solution of (4.11) corresponds to exclusively assigning the second and third subcarriers to the third link and the fourth subcarrier to the fifth link. The first subcarrier is assigned to the third link $25 \%$ of the time and to the fifth link $75 \%$ of the time.

Furthermore, by solving (4.11) for all $w_{2}^{(1)}$ and $w_{3}^{(1)}$ in the unit simplex $\left\{\left(w_{2}^{(1)}, w_{3}^{(1)}\right) \mid w_{2}^{(1)} \geq 0, w_{3}^{(1)} \geq 0, w_{2}^{(1)}+w_{3}^{(1)}=1\right\}$, we obtain in Figure 4.2 the region of all rates tuples $\left(s_{2}^{(1)}, s_{3}^{(1)}\right)$ that can be achieved by the network in Figure 4.1 for $P=10,20$ and $30 \mathrm{~dB}$. Since the problem in (4.11) is convex, it is straightforward to see that the region of rates generated by this problem is also convex, which is confirmed in Figure 4.2.

Example 2: (Average Performance) In this example, we use Mont Carlo simulations to assess the average performance of the optimal JRSPA design with timesharing in Section 4.4 and compare it with a heuristic algorithm in which the formulation in (4.9) is solved with power being equally allocated to all subcarriers. 


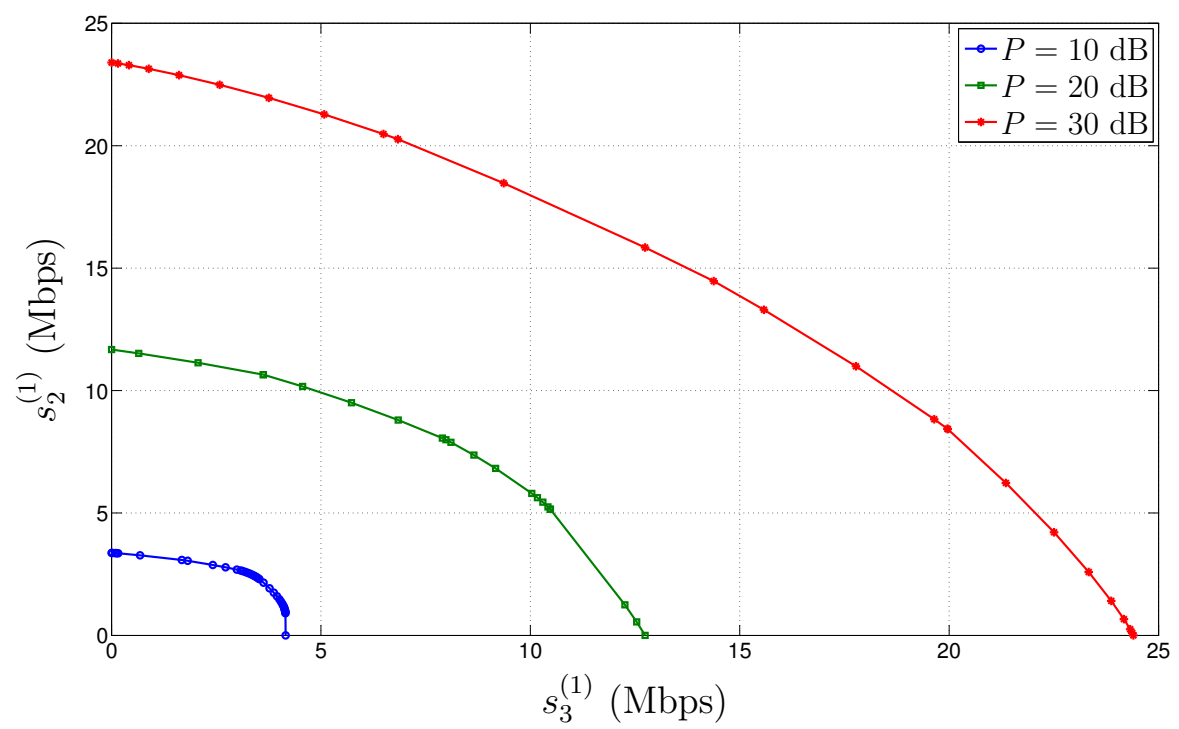

Figure 4.2: An achievable rate region for the network shown in Figure 4.1 with the channel coefficients in (4.12).

Numerical results are averaged over 100 independent realizations of a network with $N=10$ nodes. Among these nodes, five are set to be source-destination nodes, i.e., $|\mathcal{D}|=5$, and the remaining five nodes can only act as relays. All weights are set equal to one. The number of directional links is $L=90$, and the number of subcarriers available for all links is $K=4$.

The nodes of the wireless networks considered in this example are randomly dropped on a $100 \times 100 \mathrm{~m}^{2}$ square and assumed to have identical power budgets, $P_{n}=P, n=1, \cdots, N$. In accordance with the international mobile telecommunications (IMT)-Advanced document [65], the available bandwidth, $W_{0}$, is set to $20 \mathrm{MHz}$ and the thermal noise power density is set to $-174 \mathrm{dBm} / \mathrm{Hz}$. The subcarriers are assumed to be standard quasi-static frequency-flat Rayleigh fading with log-normal shadowing and pathloss components. The gains of these subcarriers are given by

$$
\left|h_{\ell k}\right|^{2}=10^{-0.1 S_{\ell}-0.1 \rho(\ell)}\left|h_{\ell k}^{\prime}\right|^{2}, \quad \forall \ell \in \mathcal{L}, \forall k \in \mathcal{K},
$$

where $S_{\ell}$ represents the shadowing component, which depends on the propagation environment urban or otherwise. Using the non line-of-sight (NLoS) model of indoor hotspot ( $\mathrm{InH})$ scenario in [65], the shadowing component, $S_{\ell}$, is assumed to be 
Gaussian-distributed in the logarithmic domain with a mean of $0 \mathrm{~dB}$ and a standard deviation of $\sigma_{s}=4 \mathrm{~dB}$. In (4.13), $\rho(\ell)$ represents the pathloss component, which depends on the length of link $\ell, d_{\ell}$, i.e., the distance between nodes $n$ and $n^{\prime}$. An expression for $\rho(\ell)$ that conforms to the InH-NLoS standard model in [65] is $\rho(\ell)=43.3 \log _{10}\left(d_{\ell}\right)+11.5+20 \log _{10}\left(f_{c}\right)$ where $f_{c}$ is the carrier frequency in $\mathrm{GHz}$ which is set to $f_{c}=3.4 \mathrm{GHz}$. The Rayleigh fading component in the channel model in (4.13) is captured by $\left|h_{\ell k}^{\prime}\right|^{2}$, where $h_{\ell k}^{\prime}$ is a zero mean unit variance complex Gaussian-distributed random variable.

The sum rates yielded by the optimal JRSPA design with time-sharing and without frequency-reuse and the heuristic algorithm are depicted in Figure 4.3 for $P$ ranging from 0 to $30 \mathrm{dBm}$. From this figure, it can be seen that the continuous scheduling design proposed in Section 4.4 significantly outperforms the heuristic algorithm.

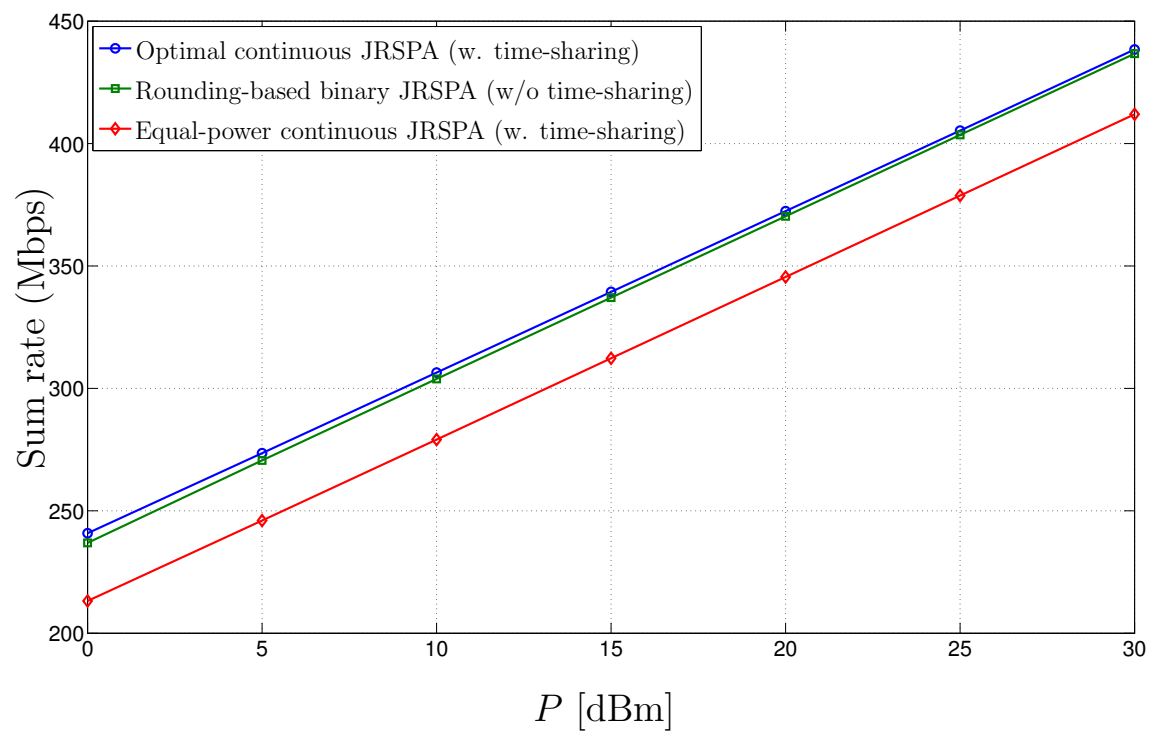

Figure 4.3: (Example 2) Sum rate generated by the optimal and heuristic JRSPA.

\subsection{Conclusion}

In this chapter we considered the joint design of routing, scheduling and power allocation in a generic wireless OFDMA-based network. By allowing the OFDMA 
subcarriers to be time shared among multiple links, the joint design problem was cast in the convex form and hence, its global optimum was found efficiently. Numerical results suggest that the continuous scheduling design proposed in this chapter significantly outperforms heuristic algorithms. 


\section{Chapter 5}

\section{JRSPA without Time-Sharing and without Frequency-Reuse}

\section{$5.1 \quad$ Introduction}

In this chapter, we consider the JRSPA problem when each subcarrier is scheduled to be used exclusively by one node during the whole signalling interval. In other words, a subcarrier, once assigned to a link, will be used by that link throughout the signalling interval. As such, subcarriers are neither allowed to be time-shared among multiple links, nor to be reused over the network.

\subsection{System Constraints}

All the constraints remain the same as explained in Section 4.2 except the scheduling constraint in (4.4). When time-sharing is not allowed, $\left\{c_{\ell k}\right\}$ assume binary values, i.e.,

$$
c_{\ell k} \in\{0,1\}, \quad \forall \ell \in \mathcal{L}, \forall k \in \mathcal{K} .
$$

It is worth noting that in the considered multicarrier structure, interference is avoided by restricting each subcarrier to be used at most once across the entire network [44]. 


\subsection{Problem Formulation}

The difference between continuous and binary JRSPA is induced by the scheduling constraints: In continuous JRSPA, the scheduling variables can take on any value in the interval $[0,1]$, whereas in the binary JRSPA, these variables can be either 0 or 1 . This difference renders solutions of the continuous JRSPA not necessarily feasible for the binary one. Having considered the continuous case in Chapter 4, in this chapter we consider the binary case, i.e., the case in which time-sharing is not allowed. In this case the JRSPA problem in (4.9) will be reformulated as

$$
\begin{gathered}
\max _{\left\{s_{n}^{(d)}\right\},\left\{x_{\ell k}^{(d)}\right\},\left\{c_{\ell k}\right\},\left\{q_{\ell k}\right\}} \sum_{d} \sum_{n, n \neq d} w_{n}^{(d)} s_{n}^{(d)}, \\
\text { subject to } \quad(4.9 \mathrm{~b})-(4.9 \mathrm{e}) \text { and }(4.9 \mathrm{~g})-(4.9 \mathrm{i}), \\
c_{\ell k} \in\{0,1\}, \quad \forall \ell \in \mathcal{L}, \forall k \in \mathcal{K} .
\end{gathered}
$$

This problem is in the form of mixed integer non-linear programming and, because of the binary constraints in $(5.2 \mathrm{~b})$, can be shown to be computationally prohibitive, even for relatively small networks. To avoid solving (5.2) directly, we will develop efficiently computable, and relatively tight, bounds on the weighted-sum rate that it yields.

Before developing these bounds, we note that, for any given set of subcarrier schedules, $\left\{c_{\ell k}\right\}$, the optimization problem in (5.2) is convex. Hence, its global optimum can be obtained by solving it for all possible choices of $\left\{c_{\ell k}\right\}$ and choosing the set that yields the maximum weighted-sum rate. The complexity of this approach is exponential in $K L$, cf. Section 5.5.

An upper bound on the weighted-sum rate yielded by (5.2) can be readily obtained by noting that the formulation in (4.11) corresponds to a relaxation of the formulation in (5.2). Since the formulation in (4.11) is convex, it can be seen that, for a given set of weights, $\left\{w_{n}^{(d)}\right\}$, it yields a rate vector that dominates the one yielded by (5.2) for the same set of weights. In other words, (4.11) yields an outer bound on the set of rates achieved by (5.2). 


\subsection{Proposed Lower Bounds}

In this section, we develop two lower bounds on the weighted-sum rate yielded by (5.2). The first lower bound can obtained by fixing any feasible set of binary schedules, $\left\{c_{\ell k}\right\}$, that satisfy (4.11g) and solving the resulting convex problem. One way to select the binary schedules is by normalizing and rounding the continuous schedules generated by the convex formulation in (4.11).

The second lower bound on the weighted-sum rate yielded by (5.2) can be obtained by inducing the effect of binary schedules through a set of constraints on the power allocations. This approach results in a new formulation that, despite being nonconvex, shares many of the features of the GP-standard form, cf. Appendix A.1, and is amenable to the monomial approximation technique in Appendix A.2. This approach and the one based on rounding will be described next.

\subsubsection{The Rounding-Based Approach}

In this approach, we consider the continuous subcarrier schedules, $\hat{c}_{\ell k} \in[0,1]$, $\forall \ell \in \mathcal{L}, \forall k \in \mathcal{K}$, obtained from the convex formulation in (4.11). To construct a set of, potentially suboptimal, binary schedules, $\left\{\tilde{c}_{\ell k}\right\}$ from continuous ones, $\left\{\hat{c}_{\ell k}\right\}$, for

every $k \in \mathcal{K}$, we set the element of $\left\{\tilde{c}_{\ell k}\right\}_{\ell=1}^{L}$ corresponding to the largest element of $\left\{\hat{c}_{\ell k}\right\}_{\ell=1}^{L}$ to 1 and the other elements to 0 ; i.e.,

$$
\tilde{c}_{\ell k}=\left\lfloor\frac{\hat{c}_{\ell k}}{\max _{\ell^{\prime} \in \mathcal{L}} \hat{c}_{\ell^{\prime} k}}\right\rfloor, \quad \forall \ell \in \mathcal{L}, \forall k \in \mathcal{K} .
$$

By construction, the elements of $\left\{\tilde{c}_{\ell k}\right\}$ satisfy the scheduling feasibility constraints in $(5.2)$, i.e., $(4.9 \mathrm{~h})$ and $(5.2 \mathrm{~b})$, and can hence be used as if they were the optimal subcarrier schedules. With $\left\{\tilde{c}_{\ell k}\right\}$ fixed, the JRSPA problem in (5.2) can be cast in the following convex form:

$$
\max _{\left\{s_{n}^{(d)}\right\},\left\{x_{\ell k}^{(d)}\right\},\left\{q_{\ell k}\right\}} \sum_{d \in \mathcal{D}} \sum_{n \in \mathcal{N}, n \neq d} w_{n}^{(d)} s_{n}^{(d)}
$$

subject to

$$
\begin{array}{ll}
\sum_{\ell \in \mathcal{L}} \sum_{k \in \mathcal{K}} a_{n \ell} x_{\ell k}^{(d)}=s_{n}^{(d)}, & \forall n \in \mathcal{N}, \forall d \in \mathcal{D}, n \neq d, \\
x_{\ell k}^{(d)} \geq 0, & \forall \ell \in \mathcal{L}, \forall k \in \mathcal{K}, \forall d \in \mathcal{D},
\end{array}
$$




$$
\begin{array}{lr}
s_{n}^{(d)} \geq 0, & \forall n \in \mathcal{N}, \forall d \in \mathcal{D}, n \neq d, \\
\sum_{d \in \mathcal{D}} x_{\ell k}^{(d)} \leq W \tilde{c}_{\ell k} \log _{2}\left(1+q_{\ell k} g_{\ell k}\right), & \forall \ell \in \mathcal{L}, \forall k \in \mathcal{K}, \\
q_{\ell k} \geq 0, & \forall n \in \mathcal{N}, \forall k \in \mathcal{K}, \\
\sum_{k \in \mathcal{K}} \sum_{\ell \in \mathcal{L}_{+}(n)} \tilde{c}_{\ell k} q_{\ell k} \leq P_{n}, & \forall n \in \mathcal{N} .
\end{array}
$$

Since the schedules $\left\{\tilde{c}_{\ell k}\right\}$ are fixed, the formulation in (5.4) can be seen to be convex. Since this formulation is based on potentially suboptimal schedules, the weighted-sum rate it generates is a lower bound on the corresponding sum rate generated by the problem with binary schedules.

\subsubsection{The GP-based Approach}

We now consider the other lower bound on the weighted-sum rate achieved by the formulation in (5.2). We begin by noting that the scheduling constraints in (5.2b) and $(4.9 \mathrm{~h})$ imply that a subcarrier $k \in \mathcal{K}$ can be used on at most one link. From (4.8), it can be seen that, when $c_{\ell k}$ is equal to zero, setting $q_{\ell k}=0$ does not incur loss of optimality. Hence, the constraints in $(5.2 \mathrm{~b})$ and $(4.9 \mathrm{~h})$ can be enforced by allowing at most one link to have a strictly positive power on any subcarrier $k \in \mathcal{K}$. In other words, the binary scheduling constraints are equivalent to

$$
q_{\ell k} q_{\ell^{\prime} k}=0, \quad \forall k \in \mathcal{K}, \forall \ell, \ell^{\prime} \in \mathcal{L}, \ell \neq \ell^{\prime}
$$

Enforcing this constraint yields to at most one 'active' link per subcarrier, which makes the recovery of the binary schedules from the power allocations straightforward.

The replacement of the constraints in (5.2b) and (4.9h) with the ones in (5.5) does not affect the flow conservation in (4.9b) and the non-negativity constraints in $(4.9 \mathrm{c})$, $(4.9 \mathrm{~d})$ and $(4.9 \mathrm{~g})$. However, this replacement affects the capacity constraints in (4.9e) and the power budget constraints in (4.9i). Since, from (5.5), only one element in $\left\{q_{\ell k}\right\}_{\ell=1}^{L}$ is strictly positive for any subcarrier $k \in \mathcal{K}$, the constraints in (4.9e) can be expressed as

$$
\sum_{d \in \mathcal{D}} x_{\ell k}^{(d)} \leq W \log _{2}\left(1+q_{\ell k} g_{\ell k}\right), \quad \forall \ell \in \mathcal{L}, \forall k \in \mathcal{K}
$$


and the constraint in (4.9i) can be expressed as

$$
\sum_{k \in \mathcal{K}} \sum_{\ell \in \mathcal{L}_{+}(n)} q_{\ell k} \leq P_{n}, \quad \forall n \in \mathcal{N}
$$

Replacing the constraints in $(4.9 \mathrm{~h}),(5.2 \mathrm{~b}),(4.9 \mathrm{e})$ and $(4.9 \mathrm{i})$ with those in $(5.5)-$ (5.7) yields an analogous formulation in which the binary schedules are accounted for by equivalent constraints on the power allocations of the links. This formulation can be expressed as

$$
\begin{array}{lr}
\max _{\left\{s_{n}^{(d)}\right\},\left\{x_{\ell k}^{(d)}\right\},\left\{q_{\ell k}\right\}} \sum_{d \in \mathcal{D}} \sum_{n \in \mathcal{N}, n \neq d} w_{n}^{(d)} s_{n}^{(d),} & \\
\text { subject to }(4.9 \mathrm{~b})-(4.9 \mathrm{~d}), & \forall \ell \in \mathcal{L}, \forall k \in \mathcal{K}, \\
\sum_{d \in \mathcal{D}} x_{\ell k}^{(d)} \leq W \log _{2}\left(1+q_{\ell k} g_{\ell k}\right), & \forall n \in \mathcal{N}, \\
\sum_{k \in \mathcal{K}} \sum_{\ell \in \mathcal{L}_{+}(n)} q_{\ell k} \leq P_{n}, & \forall \ell \in \mathcal{L}, \forall k \in \mathcal{K}, \\
q_{\ell k} \geq 0, & \forall k \in \mathcal{K}, \forall \ell, \ell^{\prime} \in \mathcal{L}, \ell \neq \ell^{\prime} . \\
q_{\ell k} q_{\ell^{\prime} k}=0, & \forall n
\end{array}
$$

The optimization problem in (5.8) is nonconvex and therefore difficult to solve. However, this problem would have been easier to solve had it been possible to ignore the ' 1 ' in the argument of the $\log (\cdot)$ function in $(5.8 \mathrm{c})$, for instance when $q_{\ell k} g_{\ell k}$ is sufficiently large [11]. Unfortunately, such an approximation is not possible in the current context because the constraints in (5.8f) imply that, while one link operates at high SNR, the others must operate at zero SNRs. Indeed, one of the tasks underlying the formulation in (5.8) is to determine which link powers ought to be set to zero, and since this information is not available a priori one cannot use the high-SNR approximation methodology in [11].

The optimization problem in (5.8), although nonconvex, is amenable to GP-based monomial approximation. In order to use this approximation, we use the exponential function to map $\left\{s_{n}^{(d)}\right\}$ and $\left\{x_{\ell k}^{(d)}\right\}$ to $\left\{t_{n}^{(d)}\right\}$ and $\left\{r_{\ell k}^{(d)}\right\}$, respectively. In particular, we have

$$
\begin{array}{ll}
s_{n}^{(d)}=\log _{2} t_{n}^{(d)}, & \forall n \in \mathcal{N}, \forall d \in \mathcal{D}, n \neq d, \\
x_{\ell k}^{(d)}=W \log _{2} r_{\ell k}^{(d)}, & \forall \ell \in \mathcal{L}, \forall k \in \mathcal{K}, \forall d \in \mathcal{D} .
\end{array}
$$


These are one-to-one mappings which enable the original variables to be readily recovered from the new ones. Using the new variables, the objective and the constraints in (5.8a)-(5.8b) can be readily expressed in GP compatible forms; cf. Appendix A.1. For the constraints in (5.8f), although the LHSs are in the form of monomials, they are not compatible with the GP framework because their RHSs are not equal to 1. This difficulty can be readily alleviated by replacing (5.5) with constraints of the form $q_{\ell k} q_{\ell^{\prime} k} \leq \epsilon$, where $\epsilon$ is a small positive number. This replacement will expand the feasible region of the considered optimization problem in (5.8). Now, using the new variables, the capacity constraints in (5.8c) can be expressed as

$$
\prod_{d \in \mathcal{D}} r_{\ell k}^{(d)} \leq 1+q_{\ell k} g_{\ell k}, \quad \forall \ell \in \mathcal{L}, \forall k \in \mathcal{K}
$$

The RHS of each constraint in (5.11) is not a monomial and hence, does not conform to the GP framework. To overcome this difficulty, we invoke the monomial approximation method described in Appendix A.2. Using this method, the expression in (A.2) is used to approximate the RHSs of (5.11) with monomial functions near some initial power allocation, $\left\{q_{\ell k}^{(0)}\right\}$.

We now consider the remaining constraints. The non-negativity constraints on the allocated powers in (5.8e) are inherently incorporated in the GP framework. Finally, the constraints in (5.8d) are compatible with the GP standard form.

Now, assuming that a feasible initial power allocation, $\left\{q_{\ell k}^{(0)}\right\}$, is given, the maximum weighted-sum rate around $\left\{q_{\ell k}^{(0)}\right\}$ can be approximated by solving the following GP:

$$
\max _{\left\{t_{n}^{(d)}\right\},\left\{r_{\ell k}^{(d)}\right\},\left\{p_{n}^{(k)}\right\}} \prod_{d \in \mathcal{D}} \prod_{n \in \mathcal{N}, n \neq d}\left(t_{n}^{(d)}\right)^{w_{n}^{(d)}}
$$

subject to

$$
\begin{array}{lr}
\prod_{\ell \in \mathcal{L}} \prod_{k \in \mathcal{K}}\left(r_{\ell k}^{(d)}\right)^{W a_{n \ell}}=t_{n}^{(d)}, & \forall n \in \mathcal{N}, \forall d \in \mathcal{D}, n \neq d, \\
t_{n}^{(d)} \geq 1, & \forall n \in \mathcal{N}, \forall d \in \mathcal{D}, n \neq d, \\
r_{\ell k}^{(d)} \geq 1, & \forall \ell \in \mathcal{L}, \forall k \in \mathcal{K}, \forall d \in \mathcal{D}, \\
\prod_{d \in \mathcal{D}} r_{\ell k}^{(d)} \leq \delta_{\ell k}\left(q_{\ell k} / q_{\ell k}^{(0)}\right)^{\theta_{\ell k},} & \forall k \in \mathcal{K}, \forall \ell \in \mathcal{L}, \\
\sum_{k \in \mathcal{K}} \sum_{\ell \in \mathcal{L}_{+}(n)} q_{\ell k} \leq P_{n}, & \forall n \in \mathcal{N},
\end{array}
$$




$$
q_{\ell k} q_{\ell^{\prime} k} \leq \epsilon, \quad \forall k \in \mathcal{K}, \forall \ell, \ell^{\prime} \in \mathcal{L}, \ell \neq \ell^{\prime}
$$

where $\delta_{\ell k}=1+q_{\ell k}^{(0)} g_{\ell k}$, and $\theta_{\ell k}=\frac{q_{\ell k}^{(0)} g_{\ell k}}{\delta_{\ell k}}$. Using a standard logarithmic transformation, (5.12) can be readily converted to an efficiently solvable convex form [9].

\subsubsection{Iterative Monomial Approximation}

Finding the global solution for the nonconvex problem in (5.8) is difficult, whereas solving the approximated problem in (5.12) is straightforward. To exploit this fact, we incorporate the formulation in (5.12) in an iterative algorithm. In particular, starting from a feasible $\left\{q_{\ell k}^{(0)}\right\}$, the problem in (5.12) is transformed into a convex form and solved using IPM. The output power allocation resulting from solving this problem is then used as the initial power allocation in the following iteration. This technique is referred to as the single condensation method and, under relatively mild conditions, is guaranteed to converge to a solution that satisfies the KKT conditions corresponding to (5.8) with (5.8f) replaced with (5.12g), cf. Appendix B. Since the problem in (5.8) with (5.8f) replaced with (5.12g) is not convex, the KKT conditions are only necessary (but not sufficient) for optimality and hence, the resulting solution is a lower bound on the achievable weighted-sum rate on the expanded feasible region. This implies that, if the initial point for the iterative monomial approximation technique happens to be appropriately chosen, the GP approach would yield the optimal design. Unfortunately, finding such an initial point depends on the network structure and is generally elusive.

\subsubsection{Obtaining a Feasible Solution}

The solution yielded by the relaxation in $(5.12 \mathrm{~g})$ is projected onto the feasible region of the original problem in (5.8). In particular, let $\left\{\hat{q}_{\ell k}\right\}$ be the power allocation attained by the iterative monomial approximation in Section 5.4.2.1. Then, the projection of $\left\{\hat{q}_{\ell k}\right\}$ onto the feasible region of (5.8) can expressed as

$$
\tilde{q}_{\ell k}=\left\lfloor\frac{\hat{q}_{\ell k}}{\max _{\ell^{\prime} \in \mathcal{L}} \hat{q}_{\ell^{\prime} k}}\right\rfloor \max _{\ell^{\prime} \in \mathcal{L}} \hat{q}_{\ell^{\prime} k}, \quad \forall \ell \in \mathcal{L}, \forall k \in \mathcal{K} .
$$

We now use $\left\{\tilde{q}_{\ell k}\right\}$, as if they were the optimal power allocations, to retrieve the subcarrier schedules. In particular, from (5.5), it can be seen that $c_{\ell k}=0$ when $q_{\ell k}=0$ and $c_{\ell k}=1$ when $q_{\ell k}>0$. Now, with $\left\{\tilde{q}_{\ell k}\right\}$ fixed, the optimal routes can be 
obtained by solving (5.8) for $\left\{x_{\ell k}^{(d)}\right\}$. In particular, $\left\{x_{\ell k}^{(d)}\right\}$ can be obtained by solving the following linear program:

$$
\begin{array}{lr}
\max _{\left\{s_{n}^{(d)}\right\},\left\{x_{\ell k}^{(d)}\right\}} \sum_{d \in \mathcal{D}} \sum_{n \in \mathcal{N} \backslash\{d\}} w_{n}^{(d)} s_{n}^{(d)}, & \\
\text { subject to } & \forall n \in \mathcal{N}, \forall d \in \mathcal{D}, n \neq d, \\
s_{n}^{(d)} \geq 0, & \forall n \in \mathcal{N}, \forall d \in \mathcal{D}, n \neq d, \\
\sum_{\ell \in \mathcal{L}} \sum_{k \in \mathcal{K}} a_{n \ell} x_{\ell k}^{(d)}=s_{n}^{(d)}, & \forall \ell \in \mathcal{L}, \forall k \in \mathcal{K}, \forall d \in \mathcal{D}, \\
x_{\ell k}^{(d)} \geq 0, & \forall \ell \in \mathcal{L}, \forall k \in \mathcal{K} . \\
\sum_{d \in \mathcal{D}} x_{\ell k}^{(d)} \leq W \log _{2}\left(1+\tilde{q}_{\ell k} g_{\ell k}\right), &
\end{array}
$$

Since this problem uses potentially suboptimal power allocations, the weighted-sum rates it generates represent a lower bound on those generated by the original problem in $(5.8)$.

\subsection{Complexity Analysis}

In this section we will examine the computational complexity required for solving the problem in (5.2) for the cases of binary scheduling, as well as the computational complexity required for computing the lower bounds in Sections 5.4.1 and 5.4.2.

The formulations for the cross-layer designs provided in Section 5.4.1 is convex and hence highly-efficient IPMs can be utilized to attain their global optimal.

Unfortunately, the log-barrier functions corresponding to the formulations in (5.4) and (5.12) are not self-concordant. Hence, to assess the complexity of solving them, we introduce auxiliary constraints in Appendix C.3 that do not affect the solution, but enables us to cast the log-barrier function in a self-concordant form. Using this approach we arrive at the following results.

Proposition 2. The complexity of solving (5.4) and (5.12) is of order $\mathcal{O}\left(\mathrm{m}^{3.5}\right)$, where

- for the lower bound on the binary JRSPA problem (Rounding-Based Approach),

$$
m=L K(3+D)+N+D(N-1) ; \quad \text { and }
$$


- for the lower bound on the binary JRSPA problem (GP-Based Approach),

$$
m=L K(3+D+(L-1) / 2)+N+D(N-1) .
$$

Using exhaustive search over the binary schedules, the complexity of solving the optimal JRSPA without time-sharing (cf. (5.2)) is bounded by

$$
\mathcal{O}\left((L+1)^{K}(L K(3+D)+N+D(N-1))^{3.5}\right) .
$$

Proof. See Appendix C.3.

Note that the GP-based lower bound is obtained by solving a sequence of GPs of the form in (5.12) iteratively; the computational complexity of each iteration is provided in the second statement of Proposition 2. Numerical results provided herein and in [11,66-69] suggest that the GP-based approach converges within 100 iterations, irrespective of the number of nodes, $N$.

Proposition 2 shows that the computational complexity required for finding the lower bounds on the optimal JRSPA without time-sharing are polynomial in the number of nodes, $N$, and subcarriers, $K$. Hence, the complexities of the lower bounds are relatively small and comparable. In particular, the computational complexity of the rounding-based lower bound on the optimal JRSPA without time-sharing grows as $K^{3.5} N^{7}$, and the corresponding complexity for the GP-based lower bound grows as $K^{3.5} N^{14}$. In contrast, the computational complexity required for finding the optimal JRSPA without time-sharing is polynomial in $N$, but exponential in $K$.

\subsection{Numerical Results}

In this section we provide numerical examples to evaluate the performance of the cross-layer design approaches presented in Sections 5.4.1 and 5.4.2 for both the rounding-based and the GP-based frameworks. We present three examples. In the first example, we apply the design techniques of Sections 5.4.1 and 5.4.2 in a 10node LTE-based network. We use the same setup as the one described in the second example of Section 4.6. We illustrate the sum rate achieved by these techniques and the corresponding routes, schedules and power allocations. In the second example, we consider the case of binary scheduling in a 4-node network. We compare the 
rounding-based and the GP-based lower bounds with the upper bound, corresponding to continuous scheduling, and the optimal binary schedules, obtained by exhaustive search. In the last example, the regions of the rates that are achievable by the design techniques in Sections 5.4.1 and 5.4.2 are plotted for an exemplary 4-node network.

Example 1: (Performance Evaluation) To illustrate the routes, schedules and power allocations generated by the techniques of Sections 5.4.1 and 5.4.2, we consider an exemplary network of $N=10$ nodes. Among these nodes, three are set to be source-destination nodes, i.e., $\mathcal{D}=\{1,2,3\}$, and the remaining seven nodes can only act as relays. To reduce the number of optimization variables and avoid overcomplicating the design, we ignore the link between any two nodes with distance greater than a threshold, $d_{T}$. For this example, $d_{T}$ is set to be half the square root of the total area, that is, for the considered example $d_{T}=50$. This setting results in reducing the number of directional links available for communication from $L=90$ to $L=42$. The number of subcarriers is assumed to be $K=8$. Providing all subcarrier gains of the considered network is not possible due to space limitations, but since these gains are dominated by the pathloss component, in Figure 5.1(a) we provide the geographic location of the nodes. In this figure, the source-destination nodes are the numbered circles, and the relay nodes are the black circles.

The cross-layer design corresponding to the network under consideration has 1703 variables of which, 336 are power variables, 336 are scheduling variables and 1131 are routing variables.

Our objective in this example is to maximize the sum rate, i.e., we set $w_{n}^{(d)}=1$, for $n, d \in\{1,2,3\}, n \neq d$. Setting $P=25 \mathrm{dBm}$, the optimal JRSPA design in (4.11) yields a sum rate of 168 Megabits per second (Mbps), the rounding-based approach in (5.4) yields a sum rate of $130 \mathrm{Mbps}$, and the GP-based approach in (5.12) yields a sum rate of $123.3 \mathrm{Mbps}$. The sequential algorithm used to obtain the GP-based lower bound is initiated at the power allocation of $\sqrt{\epsilon}$ for all $\ell \in \mathcal{L}, k \in \mathcal{K}$ and converges within 12 iterations.

In Figures 5.1(b), 5.1(c) and 5.1(d), we illustrate the routes yielded by the optimal design with time-sharing, the rounding-based binary design and the GP-based binary design, respectively. The thickness of any of the links depicted in these figures is chosen to be proportional to the aggregate data rate communicated over that link. Although the sum rates yielded by these designs are relatively close to each other, the structure of their routing, scheduling and power allocations are different. Therefore, 


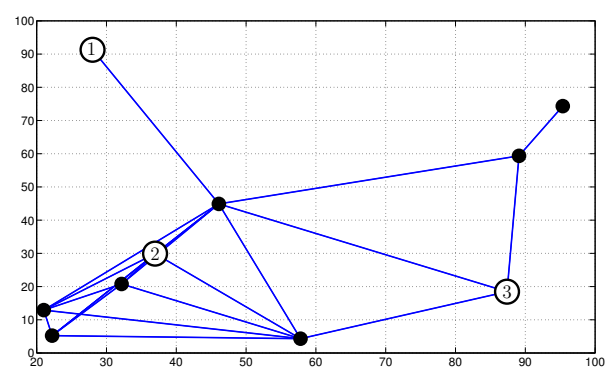

(a) Network topology

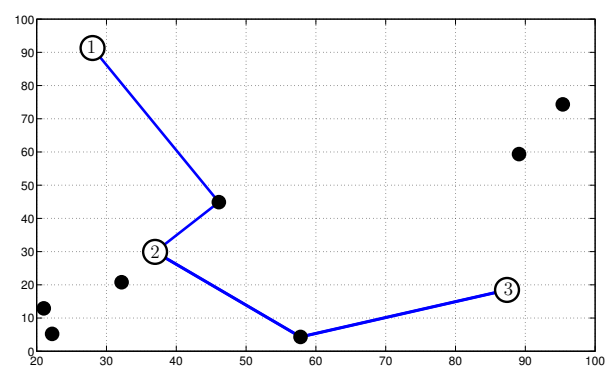

(c) Routing-Rounding-based binary (w/o timesharing)

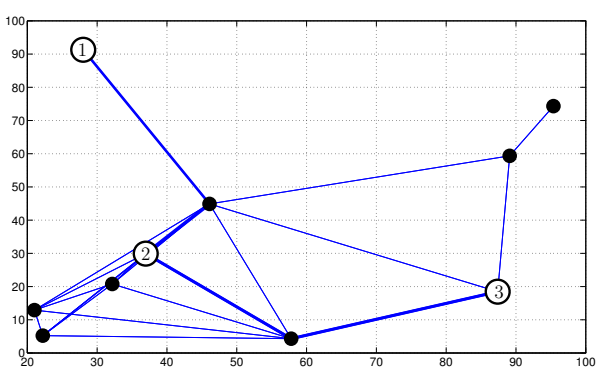

(b) Routing-Optimal continuous (w/ timesharing)

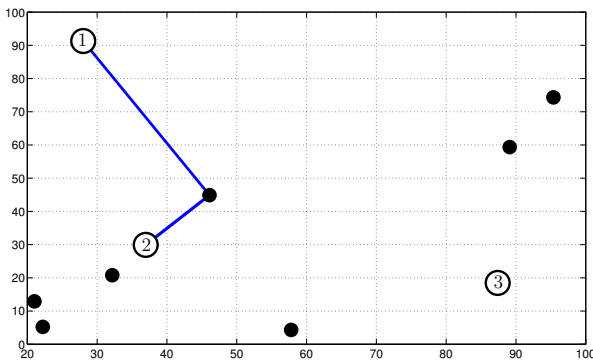

(d) Routing-GP-based binary (w/o timesharing)

Figure 5.1: The routes generated by designs considered for the 10-node network in Example 1.

it is fathomable that, for some scenarios, the structure of one of these designs may be preferred over the other ones.

In Figure 5.2, we provide a comparison between the sum rates yielded by the optimal JRSPA design with time-sharing, the rounding-based design, and the GPbased design for various node power budgets. From this figure, it can be seen that, for the considered network, the performance of the GP-based approach is better than that of the rounding-based one for values of $P$ lower than $22 \mathrm{dBm}$ and for values of $P$ higher than $22 \mathrm{dBm}$, the performance of the rounding-based approach is better than that of the GP-based one. This is because, at low values of $P$, there are fewer goodquality links and consequently fewer route alternatives. In that case, the optimal signalling strategy is likely to rely on routes with subcarrier sharing. On the other hand, at high values of $P$, there are more route alternatives and the chances of having a better performance without sharing subcarriers is higher. Hence, in that region, the performance of the rounding-based approach is likely to be superior to the 
performance of the GP-based one.

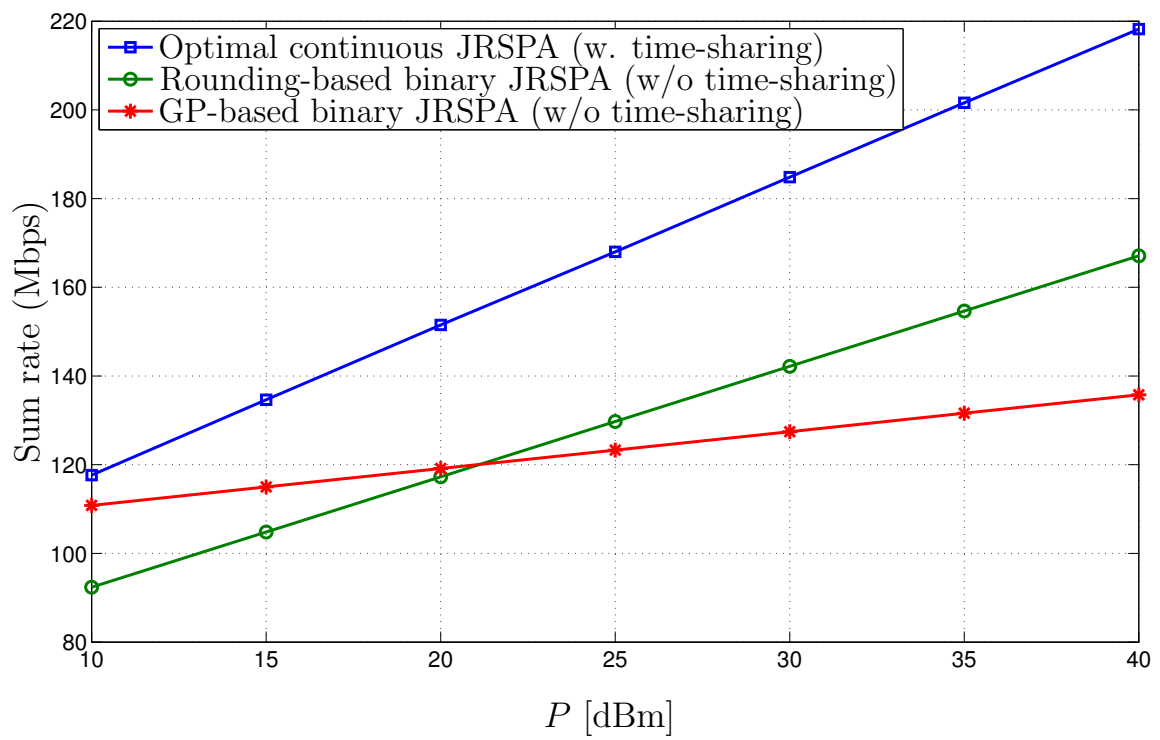

Figure 5.2: (Example 1) Sum rate generated by optimal, rounding-based and GPbased JRSPA.

Example 2: (Comparison with the Optimal Binary Solution) Since exhaustive search is computationally infeasible for large networks, to illustrate the relative tightness of the proposed bounds, in this example we compare our results with the optimal binary solution obtained through exhaustive search for a small network with $N=4$ nodes; cf. Figure 5.3. In this network, two nodes are set to be source-destination nodes, i.e., $D=\{1,2\}$ and the remaining two nodes are restricted to be relays only. The number of links and subcarriers are $L=12$ and $K=2$, respectively.

We compare the upper and lower bounds yielded by the formulations in Sections 5.4.1 and 5.4.2, respectively with the true maximum sum rate obtained by exhaustive search over all possible binary schedules, i.e., the sum rate that maximizes (5.2). This sum rate is obtained by solving a convex optimization problem analogous to the one in (5.4) for each feasible (binary) schedule. The optimal binary schedule is the one which yields the highest sum rate.

Our objective in this example is to maximize the sum rate, i.e., we set $w_{n}^{(d)}=1$, for $n, d \in\{1,2\}, n \neq d$. The rates achieved by the aforementioned schemes are plotted in Figure 5.4 for $P$ ranging from 10 to $40 \mathrm{dBm}$. This result suggests that, at low 


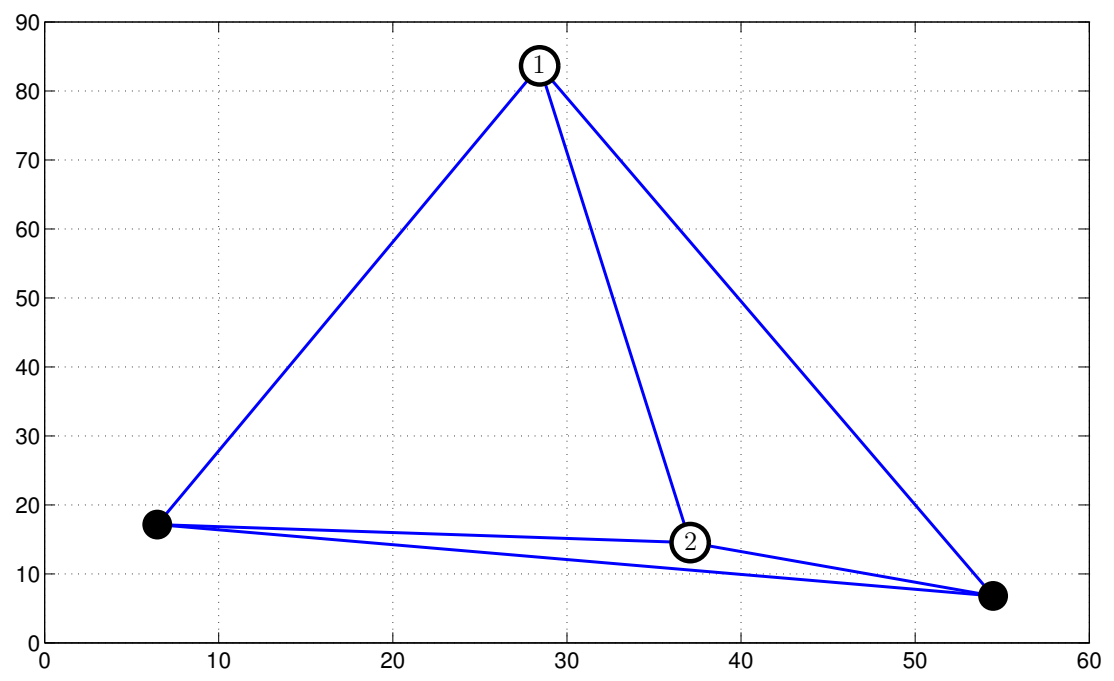

Figure 5.3: Topology of the network considered in Example 2.

values of $P$, the GP-based approach provides a higher sum rate than the roundingbased one. However, at high values of $P$, the rounding-based approach yields a higher sum rate than the GP-based one. This comes in agreement with the behaviour of the network considered in the previous example, cf. Figure 5.2. Extensive numerical experiments suggest that this behaviour is intimately related to the channel gains and network topologies. To elaborate on this observation, we note that, for the GP-based approach, the underlying iterative monomial approximation technique is known to yield a solution that satisfies the KKT conditions. This implies that this approach would yield the optimal design had the initial point been appropriately chosen. Unfortunately, finding such an initial point depends on the network structure and is generally elusive. In contrast, the rounding-based approach does not depend on an initial point. This is because this approach depends on rounding the solution of a strictly convex problem. In other words, the rounding-based approach depends solely on the network structure.

Example 3: (An achievable Rate Region) In this example, we provide the rate regions that can be achieved by the design techniques in Sections 5.4.1 and 5.4.2 for a network with $N=4$ nodes and $K=2$ subcarriers in which two nodes are sourcedestination nodes, i.e., $D=\{1,2\}$ and the remaining two nodes act as relays only. The normalized channel gain of link $\ell$ on subcarrier $k$ in $\mathrm{dB}$, i.e., $10 \log _{10} g_{\ell k}$, is given 


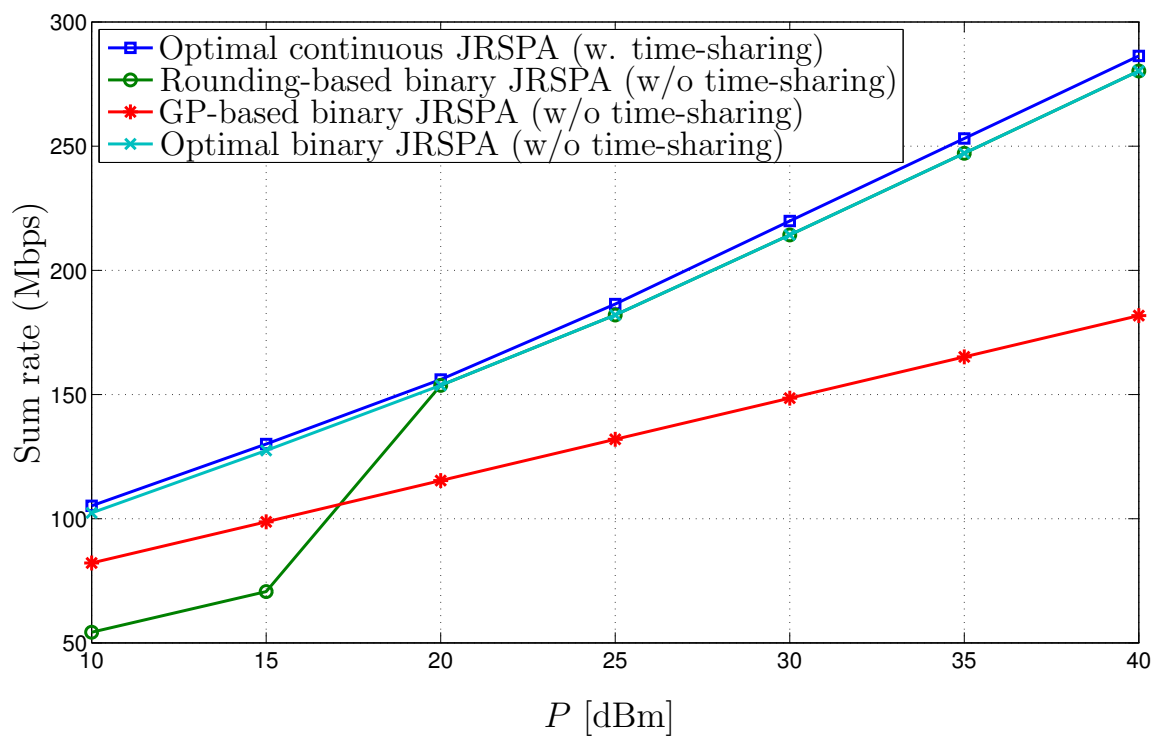

Figure 5.4: (Example 2) Sum rate generated by upper and lower bounds and optimal binary design.

Table 5.1: Channel gains $[\mathrm{dB}]$ in Example 3.

link 1 link 2 link 3 link 4 link 5 link 6 link 7 link 8 link 9 link 10 link 11 link 12

\begin{tabular}{c|cccccccccccc}
\hline subcarrier 1 & 30 & 44.7 & 24 & 24 & 47.9 & 58.3 & 4 & 49.6 & 14 & 24 & 49.9 & 41.5 \\
subcarrier 2 & 37.2 & 34 & 24 & 36.3 & 34 & 56.5 & 31.7 & 49.3 & 31.7 & 4 & 53.3 & 39.1
\end{tabular}

in Table 5.1.

Applying the cross-layer design techniques in Sections 5.4.1 and 5.4.2 for all $w_{2}^{(1)}$ and $w_{1}^{(2)}$ in the unit simplex $\left\{\left(w_{2}^{(1)}, w_{1}^{(2)}\right) \mid w_{2}^{(1)} \geq 0, w_{1}^{(2)} \geq 0, w_{2}^{(1)}+w_{1}^{(2)}=1\right\}$, we obtain the region of a set of rate tuples $\left(s_{2}^{(1)}, s_{1}^{(2)}\right)$ that can be achieved over the considered network. For $P=20 \mathrm{dBm}$, these regions are shown in Figure 5.5. Since the optimal continuous JRSPA design can be cast in a convex from that involves the pair $\left(s_{2}^{(1)}, s_{1}^{(2)}\right)$, it is straightforward to see that the region of rate tuples $\left(s_{2}^{(1)}, s_{1}^{(2)}\right)$ generated by this design is also convex for any given network. This comes from the fact that the feasible region of a convex problem is also convex. It can be seen from the figure that the optimal continuous JRSPA design yields a greater rate region than the GP-based and the rounding-based approaches. Also it can be seen that, in comparison with the rounding-based approach, the GP-based approach yields larger 
values of $s_{1}^{(2)}$ for some values of $s_{2}^{(1)}$, whereas, for other values of $s_{2}^{(1)}$, it yields smaller values of $s_{1}^{(2)}$.

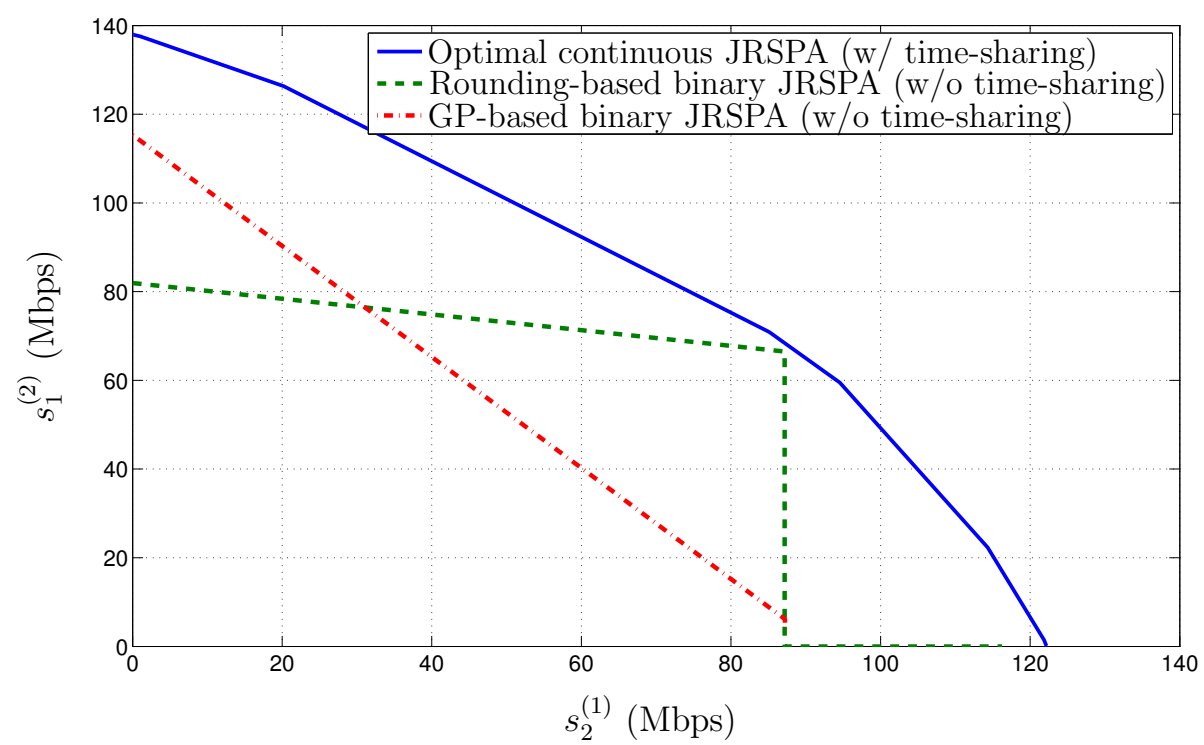

Figure 5.5: An achievable rate region for the network in Example 3 with the channel gains in Table 5.1.

\subsection{Conclusion}

In this chapter we considered the joint design of data routes, binary schedules and power allocations in a generic OFDMA-based network. We developed two efficiently computable lower bounds on the maximum weighted sum of the rates that can be reliably communicated over this network. The first bound is based on a GP approximation of the original design problem, whereas the second bound is based on normalizing and rounding the solution of a relaxed version thereof. Numerical investigations suggest that the gap between these lower bounds and the upper bound is generally small and decreases with the increase in the power budgets. 


\section{Chapter 6}

\section{JRSPA without Time-Sharing and with Frequency-Reuse}

\subsection{Introduction}

In this chapter, we consider the JRSPA problem when each subcarrier can be scheduled to be used by several nodes across the network during the whole signalling interval. As such, subcarriers are subjected to reuse but not to time-sharing. We will provide a characterization of the constraints that must be satisfied by the design variables. Using this characterization, we will formulate the network design as an optimization problem, which although nonconvex, will be transformed into a form that is more convenient for obtaining locally optimal solutions efficiently. This transformation enables us to develop an iterative GP-based algorithm whose convergence to a point that satisfies the KKT conditions is guaranteed.

\subsection{System Constraints}

In this section we characterize the constraints that feasible routes and power allocations must satisfy.

\subsubsection{Routing Constraints}

The constraints that must be satisfied by the routing variables, $\left\{x_{\ell k}^{(d)}\right\}$, are identical to those described in (4.1)-(4.3). 


\subsubsection{Power Allocation Constraints}

Consider the requirement for the network not to operate in broadcasting mode, i.e., a node cannot simultaneously broadcast to multiple destinations on the same subcarrier. In other words, of all the links in $\mathcal{L}_{+}(n)$, only one element in the set $\left\{q_{\ell k}\right\}_{\ell \in \mathcal{L}_{+}(n)}, \forall n \in \mathcal{N}, k \in \mathcal{K}$, can assume a strictly positive value. This constraint can be represented as

$$
a_{n \ell}^{+} a_{n \ell^{\prime}}^{+} q_{\ell k} q_{\ell^{\prime} k}=0, \quad \ell, \ell^{\prime} \in \mathcal{L}, k \in \mathcal{K}, n \in \mathcal{N}
$$

where $a_{n \ell}^{+}=\max \left\{0, a_{n \ell}\right\}$, that is, $a_{n \ell}^{+}=1$ if $\ell \in \mathcal{L}_{+}(n)$ and zero otherwise.

We now consider the requirement for the network to operate in a half-duplex mode. Since in the current network, no scheduling is considered, this requirement can be imposed by restricting the nodes to transmit and receive data on distinct subcarriers. In other words, if the transmission power on an incoming link of node $n$ on subcarrier $k$ is positive, then the power allocated by node $n$ to subcarrier $k$ of its outgoing links must be zero. Using the link powers defined in (3.4), the half-duplex operation can be captured by the following set of constraints:

$$
a_{n \ell}^{-} a_{n \ell^{\prime}}^{+} q_{\ell k} q_{\ell^{\prime} k}=0, \quad \ell, \ell^{\prime} \in \mathcal{L}, k \in \mathcal{K}, n \in \mathcal{N}
$$

where $a_{n \ell}^{-}=\left|\min \left\{0, a_{n \ell}\right\}\right|$, that is, $a_{n \ell}^{-}=1$ if $\ell \in \mathcal{L}_{-}(n)$ and zero otherwise. Note that (6.1) and (6.2) are trivially satisfied if either link $\ell$ or $\ell^{\prime}$ are not connected to node $n$.

In a practical network, the nodes are likely to have a certain power budget which bounds the total power allocated by each node on all subcarriers. This constraint can be written as

$$
\sum_{k \in \mathcal{K}} p_{n}^{(k)} \leq P_{n}, \quad n \in \mathcal{N}
$$

Using (3.4b), this constraint can be cast as

$$
\sum_{k \in \mathcal{K}} \sum_{\ell \in \mathcal{L}} a_{n \ell}^{+} q_{\ell k} \leq P_{n}, \quad n \in \mathcal{N}
$$

where the link powers must satisfy the following non-negativity constraints:

$$
q_{\ell k} \geq 0, \quad \ell \in \mathcal{L}, k \in \mathcal{K} .
$$




\subsubsection{Capacity Constraints}

To complete the characterization of the network, we point out that the data flows and the power allocations are coupled by the maximum aggregate rate that can be supported by the subcarriers of each link. In particular, the aggregate rate $\sum_{d \in \mathcal{D}} x_{\ell k}^{(d)}$ must not exceed the capacity of the $k$-th subcarrier of link $\ell$. As mentioned in Section 3.3, when the nodes use Gaussian signalling, this capacity is given by $W \log _{2}(1+\operatorname{SINR}(\ell, k))$, where SINR is defined in (3.3). For notational convenience, we will use the fact that each link $\ell$ originates at node $n$ and ends at node $n^{\prime}$. Using this notation and invoking (3.3), the constraint on the aggregate rate on each subcarrier $k \in \mathcal{K}$ of each link $\ell \in \mathcal{L}$ can be written in terms of $\left\{q_{\ell k}\right\}$ as

$$
\sum_{d} \frac{x_{\ell k}^{(d)}}{W} \leq \log _{2}\left(1+\frac{q_{\ell k} g_{\ell k}}{1+\sum_{\ell^{\prime} \in \mathcal{L} \backslash\{\ell\}} q_{\ell^{\prime} k} g_{\ell^{\prime \prime} k}}\right),
$$

where, here and henceforth, $\ell^{\prime \prime}$ is used to denote the index of the link connecting the node at which link $\ell^{\prime}$ originates to the node at which link $\ell$ ends, i.e., node $n^{\prime}$.

\subsection{Problem Formulation}

We are now ready to formulate the joint design as an optimization problem. To ensure the feasibility of the rates generated by our design, the constraints on the routes in (4.1)-(4.3) and on power allocations in (3.4)-(6.5) must be satisfied. Hence, the design problem can be written as

$$
\max _{\left\{s_{n}^{(d)}\right\},\left\{x_{\ell k}^{(d)}\right\},\left\{q_{\ell k}\right\}} \sum_{d \in \mathcal{D}} \sum_{n \in \mathcal{N} \backslash\{d\}} w_{n}^{(d)} s_{n}^{(d)}
$$

subject to

$$
\begin{array}{lr}
\sum_{\ell \in \mathcal{L}} \sum_{k \in \mathcal{K}} a_{n \ell} x_{\ell k}^{(d)}=s_{n}^{(d)}, & n \in \mathcal{N} \backslash\{d\}, d \in \mathcal{D}, \\
x_{\ell k}^{(d)} \geq 0, & \ell \in \mathcal{L}, k \in \mathcal{K}, d \in \mathcal{D}, \\
s_{n}^{(d)} \geq 0, & n \in \mathcal{N} \backslash\{d\}, d \in \mathcal{D}, \\
\sum_{d \in \mathcal{D}} \frac{x_{\ell k}^{(d)}}{W} \leq \log _{2}\left(1+\frac{q_{\ell k} g_{\ell k}}{1+\sum_{\ell^{\prime} \in \mathcal{L} \backslash\{\ell\}} q_{\ell^{\prime} k} g_{\ell^{\prime \prime} k}}\right), & k \in \mathcal{K}, \ell \in \mathcal{L}, \\
q_{\ell k} \geq 0, & \ell \in \mathcal{L}, k \in \mathcal{K},
\end{array}
$$




$$
\begin{array}{lr}
\sum_{k \in \mathcal{K}} \sum_{\ell \in \mathcal{L}} a_{n \ell}^{+} q_{\ell k} \leq P_{n}, & n \in \mathcal{N}, \\
a_{n \ell}^{-} a_{n \ell^{\prime}}^{+} q_{\ell k} q_{\ell^{\prime} k}=0, & \ell, \ell^{\prime} \in \mathcal{L}, k \in \mathcal{K}, n \in \mathcal{N}, \\
a_{n \ell}^{+} a_{n \ell^{\prime}}^{+} q_{\ell k} q_{\ell^{\prime} k}=0, & \ell, \ell^{\prime} \in \mathcal{L}, k \in \mathcal{K}, n \in \mathcal{N} .
\end{array}
$$

The optimization problem in (6.6) is nonconvex because the RHS of the capacity constraints in (6.6e) is the logarithm of a rational function of $\left\{q_{\ell k}\right\}$, and therefore not concave. This can be verified by showing that the corresponding Hessian matrices are non-definite. The equality constraints in (6.6h) and (6.6i) are not affine and hence, also nonconvex.

By examining (6.6), it can be seen that this problem shares some features with the GP standard form in Appendix A.1, including the non-negativity of the optimization variables and the product form appearing in some of the constraints. To exploit this observation, in the next section we will perform a change of variables that will enable us to express the objective and all, but one set, of constraints in a GP-compatible form. The residual constraints that do not comply with the GP standard form are approximated using the monomial approximation in Appendix A.2. Under relatively mild conditions [10], iterative application of this technique is known to yield a local solution of the KKT system corresponding to (6.6), see e.g., [56].

\subsection{Proposed GP-based Algorithm}

To cast (6.6) in a form that is amenable to an efficient approximation technique, we use (5.9) and (5.10) to transform $\left\{s_{n}^{(d)}\right\}$ and $\left\{x_{\ell k}^{(d)}\right\}$ to $\left\{t_{n}^{(d)}\right\}$ and $\left\{r_{\ell k}^{(d)}\right\}$, respectively.

Using (5.9) and (5.10), the objective in (6.6a) and the constraints in (6.6b)-(6.6d) can be readily expressed in a GP-compatible form. Substituting from (5.10) into the constraints in (6.6e) yields the following set of equivalent constraints:

$$
\left(1+\sum_{\ell^{\prime} \in \mathcal{L} \backslash\{\ell\}} q_{\ell^{\prime} k} g_{\ell^{\prime \prime} k}\right) \prod_{d \in \mathcal{D}} r_{\ell k}^{(d)} \leq 1+\sum_{\ell^{\prime} \in \mathcal{L}} q_{\ell^{\prime} k} g_{\ell^{\prime \prime} k}, \quad k \in \mathcal{K}, \ell \in \mathcal{L} .
$$

The RHS of (6.7) is not a monomial, which renders (6.7) not compatible with the GP framework described in Appendix A.1. To provide a GP-compatible approximation of the cross layer design in (6.6), we will invoke (A.2) in Appendix A.2 to approximate the RHS of (6.7) by a monomial expression near an initial $\left\{q_{\ell k}^{(0)}\right\}$, where $\left\{q_{\ell k}^{(i)}\right\}$ is the 
power allocation at the $i$-th iteration.

We now consider the constraints in (6.6h) and (6.6i). The RHSs of these constraints are zero, which makes them incompatible with the GP framework in (A.1). This problem can be alleviated by constraining their LHSs to be less than an arbitrary small number $\epsilon>0$.

Using the transformations in (5.9) and (5.10), the monomial approximation of (6.7), and the relaxed versions of (6.6h) and (6.6i), the joint design of data routes and power allocations in (6.6) can be approximated with the following GP:

$$
\max _{\left\{t_{n}^{(d)}\right\},\left\{r_{\ell k}^{(d)}\right\},\left\{q_{\ell k}\right\}} \prod_{d \in \mathcal{D}} \prod_{n \in \mathcal{N} \backslash\{d\}}\left(t_{n}^{(d)}\right)^{w_{n}^{(d)}},
$$

subject to

$$
\begin{array}{lr}
\prod_{\ell \in \mathcal{L}} \prod_{k \in \mathcal{K}}\left(r_{\ell k}^{(d)}\right)^{W a_{n \ell}}=t_{n}^{(d)}, & n \in \mathcal{N} \backslash\{d\}, d \in \mathcal{D}, \\
r_{\ell k}^{(d)} \geq 1, & \ell \in \mathcal{L}, k \in \mathcal{K}, d \in \mathcal{D}, \\
t_{n}^{(d)} \geq 1, & n \in \mathcal{N} \backslash\{d\}, d \in \mathcal{D}, \\
\left(1+\sum_{\ell \mathcal{L} \backslash\{\ell\}} q_{\ell^{\prime} k} g_{\ell^{\prime \prime} k}\right) \prod_{d \in \mathcal{D}} r_{\ell k}^{(d)} \leq \rho_{\ell k} \prod_{\ell^{\prime} \in \mathcal{L}}\left(q_{\ell^{\prime} k} / q_{\ell^{\prime} k}^{(0)}\right)^{\eta_{\ell^{\prime} k}}, & k \in \mathcal{K}, \ell \in \mathcal{L}, \\
\sum_{k \in \mathcal{K}} \sum_{\ell \in \mathcal{L}} a_{n \ell}^{+} q_{\ell k} \leq P_{n}, & n \in \mathcal{N}, \\
a_{n \ell}^{-} a_{n \ell^{\prime}}^{+} q_{\ell k} q_{\ell^{\prime} k} \leq \epsilon, & \ell, \ell^{\prime} \in \mathcal{L}, k \in \mathcal{K}, n \in \mathcal{N}, \\
a_{n \ell}^{+} a_{n \ell^{\prime}}^{+} q_{\ell k} q_{\ell^{\prime} k} \leq \epsilon, & \ell, \ell^{\prime} \in \mathcal{L}, k \in \mathcal{K}, n \in \mathcal{N},
\end{array}
$$

where $\rho_{\ell k}=\left(1+\sum_{\ell^{\prime} \in \mathcal{L}} q_{\ell^{\prime} k}^{(0)} g_{\ell^{\prime \prime} k}\right)$, and $\eta_{\ell^{\prime} k}=q_{\ell^{\prime} k}^{(0)} g_{\ell^{\prime \prime} k} / \rho_{\ell k}$. Note that the non-negativity constraints in (6.6f) are inherently incorporated in the GP framework.

Using the standard exponential transformation in Appendix C.3.2, the GP in (6.8) can be readily transformed into a convex optimization problem which can be solved in polynomial time using highly efficient IPMs [9]. Hence, (6.8) enables us to efficiently solve the cross layer design problem approximately in the neighbourhood of any initial set $\left\{q_{\ell k}^{(0)}\right\}$.

Finding the global solution for the nonconvex problem in (6.6) is difficult, whereas solving the approximated problem in (6.8) is straightforward. To exploit this fact, we use the single condensation method, whereby the output of solving (6.8) for an initial 
set $\left\{q_{\ell k}^{(0)}\right\}$ is used as a starting point for the subsequent iteration [56,68]. Recall that under relatively mild conditions, the convergence of this method to a solution of the KKT system corresponding to (6.6) is guaranteed [10].

The relaxations in $(6.8 \mathrm{~g})$ and $(6.8 \mathrm{~h})$ may result in infeasible power allocations that do not satisfy the constraints in (6.1) and (6.2). To construct a feasible, but potentially suboptimal, power allocations, the elements of $\left\{q_{\ell k}\right\}$ that are less than or equal to $\sqrt{\epsilon}$ are set to zero. More specifically,

$$
q_{\ell k} \leftarrow \begin{cases}0 & \text { if } q_{\ell k} \leq \sqrt{\epsilon} \\ q_{\ell k} & \text { otherwise }\end{cases}
$$

After updating the link powers according to the rule in (6.9), the node powers can be readily obtained using $(3.4 \mathrm{~b})$. With $\left\{q_{\ell k}\right\}$ fixed, the optimization problem in (6.6) becomes linear and thereby, the optimal routes and input rates can be readily obtained.

\subsection{Complexity Analysis}

In this section we examine the computational complexity required for solving the JRSPA problem with frequency-reuse but without time-sharing. The algorithms in Section 6.4 solves the families of the optimization problems in (6.8), iteratively. Each of these problems is in the form of a GP that can be readily converted to a convex optimization problem and can be efficiently solved using IPM-based solvers.

The log-barrier functions related to the problems in (6.8) is not self-concordant. To circumvent this difficulty, in Appendix C.4 we introduce a set of auxiliary variables and constraints which, although redundant, enable us to construct self-concordant logbarrier functions. Using these functions and the results in [8], we have the following proposition for the computational complexity of solving the problem in (6.8).

Proposition 3. The complexity of solving (6.8) is of order

$$
\mathcal{O}\left((L K(3 L+2)+N+D(N-1))^{3.5}\right)
$$

Proof. See Appendix C.4. 
Proposition 3 shows that the complexity of solving the problem in (6.8) is polynomial in the number of nodes, $N$, and the number of subcarriers, $K$. In particular, it grows as $L^{7} K^{3.5}$.

\subsection{Numerical Results}

In this section we provide numerical results to evaluate the performance of joint routing and resource allocation algorithms for the case with frequency-reuse but without time-sharing. For this case, we provide illustrative examples to demonstrate the advantage of the designs in which each subcarrier can be used simultaneously by multiple links over those in which each subcarrier can be used by only one link at any given time instant.

The results reported herein are obtained using the CVX package [62] with an underlying MOSEK solver [64].

Example 1: (Performance Evaluation) Consider an exemplary network with $N=6$ nodes. In this network, nodes 3 and 4 wish to communicate with nodes 2 and 1 , respectively, over $K=4$ subcarriers. In particular, for destination node $d=1$, node $n=4$ is the source and the other nodes, i.e., $\{2,3,4,5\}$ are potential relays, and, for destination node $d=2$, node $n=3$ is the source and nodes $\{1,4,5,6\}$ are potential relays.

In this example, we use the same setup as the one described in the second example of Section 4.6 except that the locations of the nodes are randomly generated over a $300 \times 300 \mathrm{~m}^{2}$ square and the bandwidth around each subcarrier is set to $W=200 \mathrm{KHz}$.

The considered network has $L=30$ directional links and therefore the channel matrix has $30 \times 4$ elements. For space considerations, this matrix is not provided, but since the channel coefficient on each subcarrier is dominated by the pathloss component, we provide the coordinates of the nodes in $300 \times 300 \mathrm{~m}^{2}$ square from which calculating pathloss components is straightforward. The coordinates of the nodes are $\{(283,202),(191,208),(287,20),(72,76),(201,67),(86,200)\}$.

Setting the node power budgets to $P=20 \mathrm{dBm}$ and assuming that both rates have equal weights, $w_{3}^{(2)}=w_{4}^{(1)}=1$, the joint design algorithm in Section 6.4 yields a sum-rate of $9.1 \mathrm{bits} / \mathrm{s} / \mathrm{Hz}$. The data routes and power allocations obtained by this algorithm are shown in Figure 6.1 and Table 6.1, respectively. As shown in Figure 6.1, subcarrier $k=1$ is used twice and due to the half-duplex constraint, transmission and 


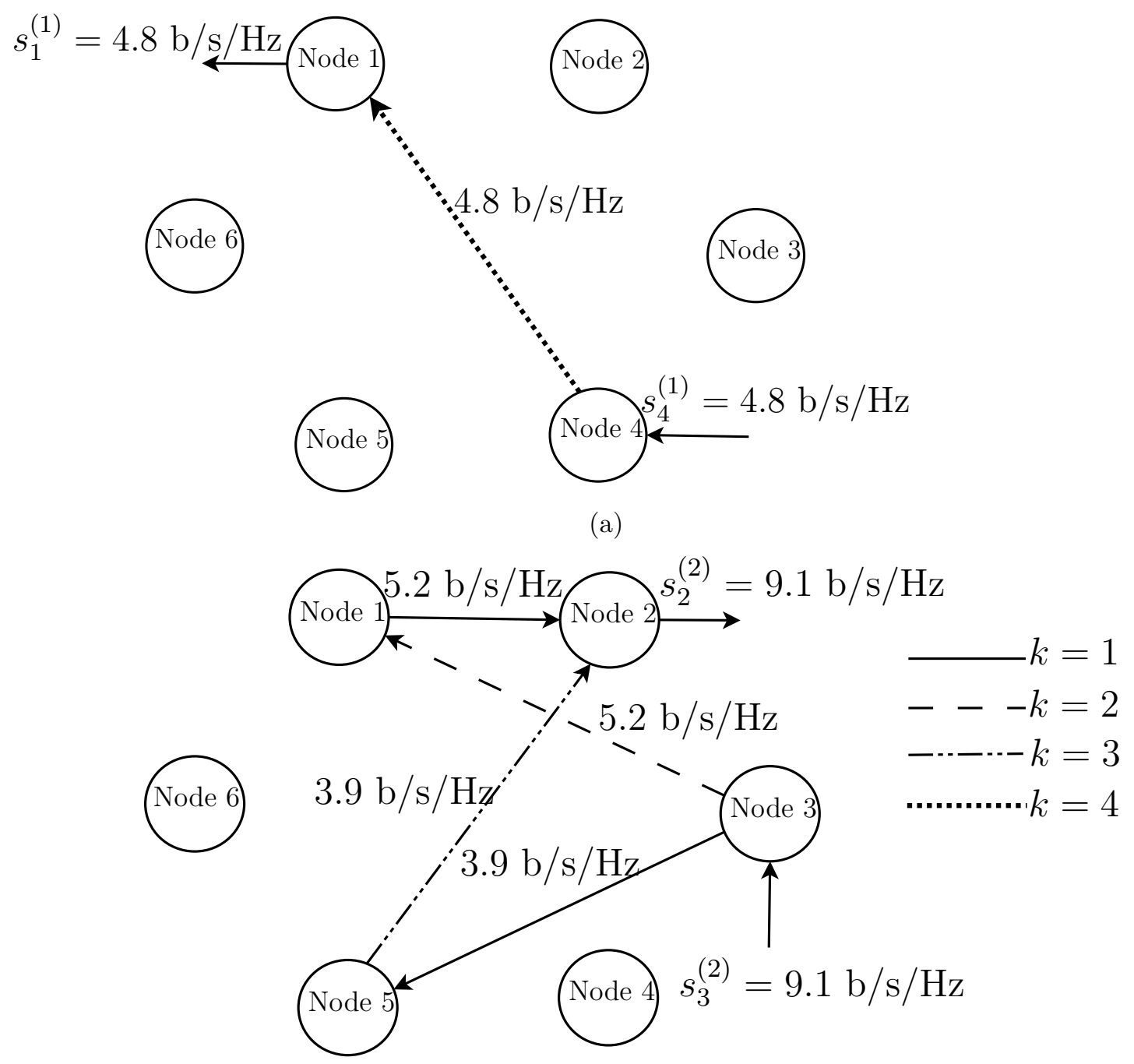

(b)

Figure 6.1: Data routes for (a) $d=1$, (b) $d=2$ in Example 1.

reception take place over distinct subcarriers at each node. We will later show the advantage of the proposed algorithm in extracting the potential of reusing subcarriers over the algorithms in which frequency-reuse is not considered.

Example 2: (Sum Rate Comparison) In this example, we consider maximizing the sum rate that can be reliably communicated by the quasi-static network in Figure 6.2. This network has $N=4$ nodes, which were randomly dropped in a square of $100 \times$ $100 \mathrm{~m}^{2}$. The number of links is $L=12$, and the number of destinations is $D=2$; i.e., $\mathcal{D}=\{1,2\}$. Two subcarriers are available for communication, i.e., $K=2$. We 
Table 6.1: Power allocations $(\mathrm{mW})$ in Example 1.

\begin{tabular}{|l|l|l|l|}
\hline$n=1$ & $n=3$ & $n=4$ & $n=5$ \\
\hline$q_{1,1}=12$ & $q_{11,2}=45$ & $q_{16,4}=100$ & $q_{22,3}=25$ \\
& $q_{14,1}=55$ & & \\
\hline
\end{tabular}

Table 6.2: Channel gains in Example 2.

\begin{tabular}{c|ccccccccccccc} 
& link 1 & link 2 & link 3 & link 4 & link 5 & link 6 & link 7 & link 8 & link 9 & link 10 & link 11 & link 12 \\
\hline subcarrier 1 & 10 & 9.2 & 10 & 9.6 & 9.8 & 10.8 & 11.1 & 9.1 & 10.7 & 8.5 & 11.4 & 12 \\
subcarrier 2 & 8.5 & 11 & 9.3 & 9.3 & 9.4 & 10.2 & 12.1 & 10 & 11.3 & 9.1 & 10.3 & 12
\end{tabular}

obtained the realization of the channel gains represented in Table 6.2. For ease of exposition, the $(\ell, k)$-th entry of this table represents $\log _{10}\left(g_{\ell k}^{-1}\right)$ instead of $g_{\ell k}$.

Setting $P=20 \mathrm{dBm}$, the algorithm in Section 6.4 yielded a sum rate of 284.2 Mbps with the 'active' links represented by the thicker solid lines in Figure 6.2; dashed lines represent 'inactive' links. As shown in this figure, each subcarrier has been used twice during the signalling interval. It is worth noting that even for the small network considered in this example, the number of variables and constraints is relatively large; 80 variables and 172 constraints.

To demonstrate the benefit of the proposed algorithm, we compare its sum rate performance with binary and continuous scheduling-based approaches. Both approaches do not allow for subcarrier reuse and ensure that, at any given time, each subcarrier is used by one node only. In the binary approach, each subcarrier is assigned to a certain node for the entire signalling interval. As such, binary scheduling can be regarded as a special case of the general cross layer design considered herein. In this case, the cross layer design problem corresponds to a mixed integer program in (5.2), and can be solved using an exhaustive search over $\frac{L !}{(L-K) !}$ possible binary schedules; a computationally prohibitive approach for large networks. Performing this search for the network in Figure 6.2 yielded a maximum sum rate of 


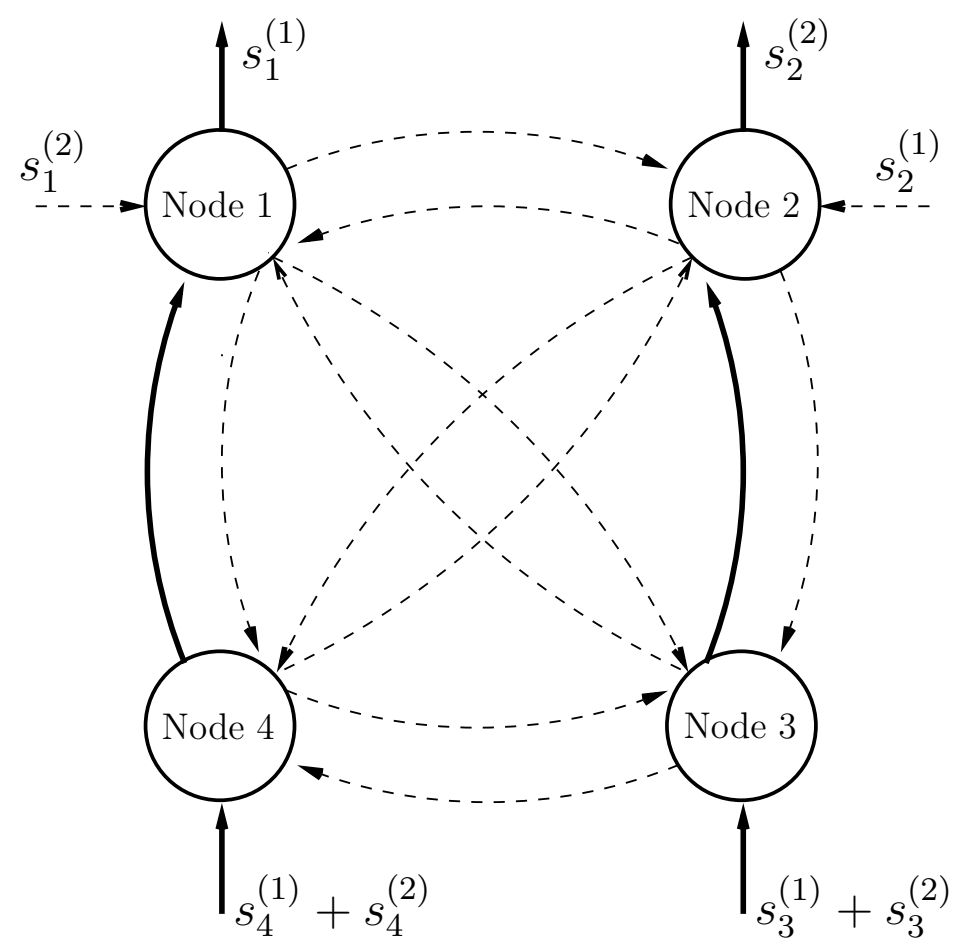

Figure 6.2: Network schematic. Each link is composed of two subcarriers.

257.1 Mbps. In addition to binary scheduling, we also compare the sum rate performance of the proposed approach with that of continuous scheduling, which subsumes binary scheduling and allows each subcarrier to be time-shared by multiple nodes. This type of scheduling was shown in Chapter 4 to give rise to a convex optimization problem that can be efficiently solved. Using the formulation in Section 4.4, this type of scheduling yielded a maximum sum rate of 262.5 Mbps.

Performing the exhaustive search required for binary scheduling at various values of $P$ is computationally expensive. Hence, for that case, we only provide a sum rate comparison between the approach proposed herein and the one based on continuous (time-sharing) scheduling. This comparison is depicted in Figure 6.3, which shows that in this scenario the cross layer design proposed herein can significantly outperform the scheduling-based approach. For instance, at a sum rate of $200 \mathrm{Mbps}$, the proposed approach provides a power gain of $8 \mathrm{dBm}$. As $P$ increases, the effect of interference becomes significant and reuse becomes less effective. This effect would not arise had the network had a clustered structure.

Example 3: (Sum Rate Comparison) To further demonstrate the benefit of the 


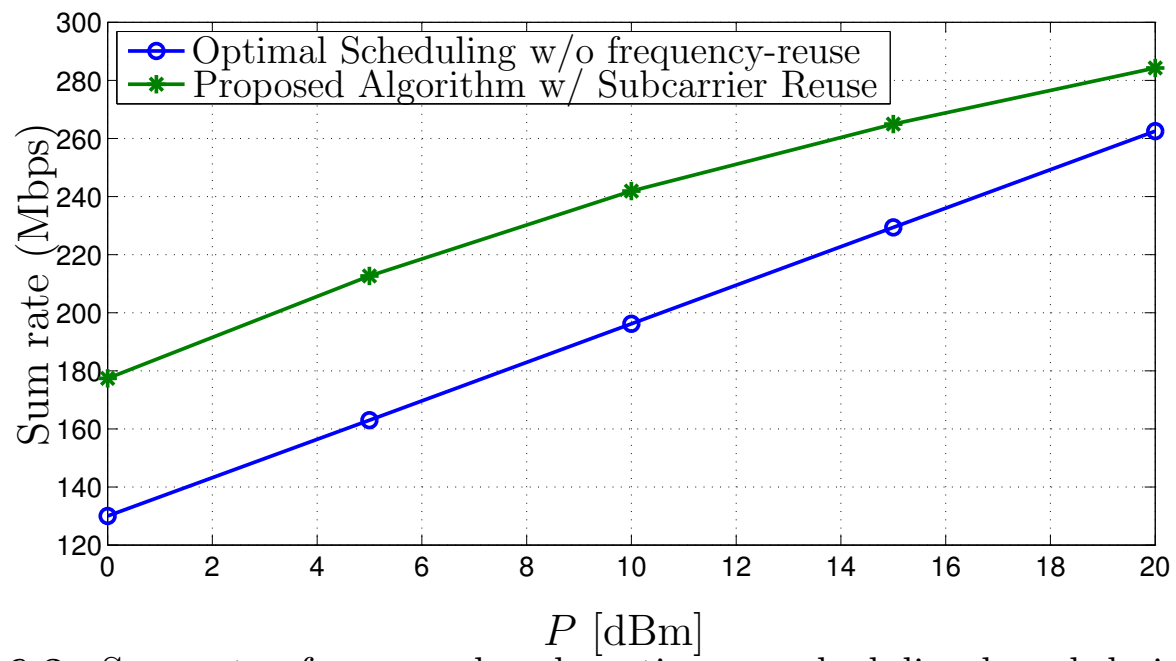

Figure 6.3: Sum rate of proposed and continuous scheduling-based designs for the network considered in Example 2.

proposed algorithm over scheduling-based designs even in non cluster-like structures, in this example we consider a network of $N=8$ nodes among which four nodes are set to be destination nodes, i.e., $D=4$. We consider the same scenario as described in Example 2. The network has $L=56$ links and operates over $K=4$ subcarriers. The number of variables for the considered network in this example is 1148 and the number of constraints is 3424 .

Providing the subcarrier gains of the considered network is not possible due to space limitations. However, since these gains are dominated by the pathloss component, in Figure 6.4 we provide the geographic location of the nodes. Implementing the algorithm in Section 6.4 for $P=20 \mathrm{dBm}$ yields the routes shown in Figure 6.4. In this figure, the thickness of the lines are made proportional to the power allocated to the transmissions of the nodes. It can be seen from this figure that each subcarrier is reused over multiple links. This is in contrast with the scheduling-based design, which allows each subcarrier to be used at most once.

The sum rates achieved by the algorithm in Section 6.4 and the approach based on continuous (time-sharing) scheduling are provided in Figure 6.5 for various $P$. From this figure, it can be seen that, despite being potentially suboptimal, at a power budget of $20 \mathrm{dBm}$ per node, the proposed design yields a throughput advantage in excess of 120 Mbps over the design based on the optimal continuous scheduling. 


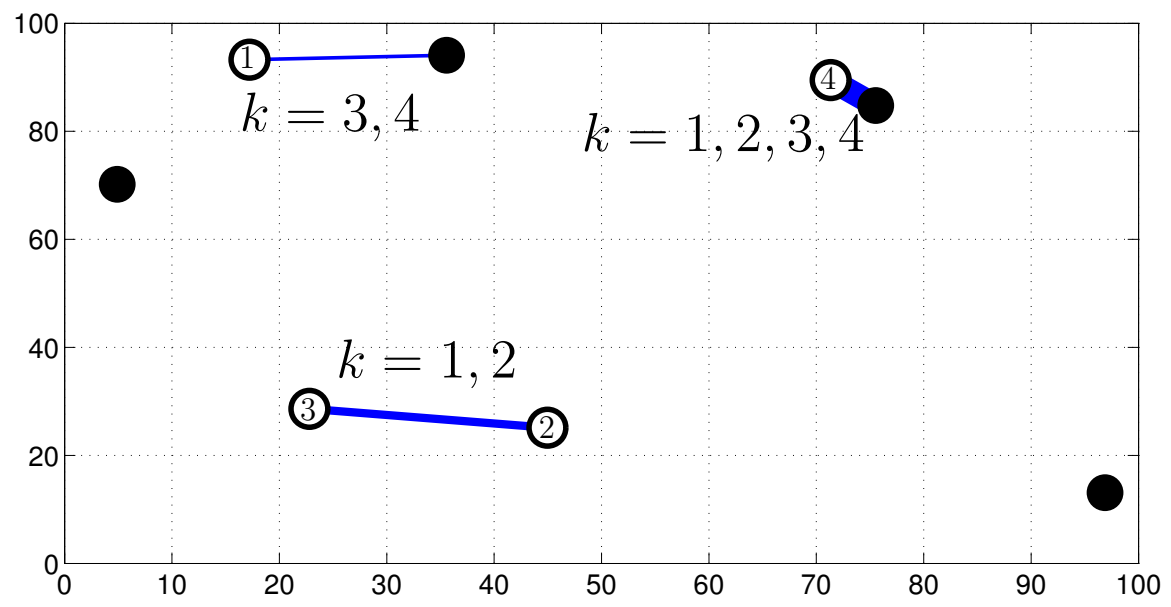

Figure 6.4: Schematic of the network in Example 3, data routes, subcarriers reuse and corresponding power allocations.

As the network size increases, the opportunity of reusing a subcarrier also increases and therefore the advantage of using the proposed algorithm over scheduling-based techniques becomes more pronounced.

\subsection{Conclusion}

In this chapter, we considered the joint design of data routes and power allocations in a generic OFDMA-based wireless network in which each frequency subcarrier can be used by multiple nodes simultaneously. We developed an efficient iterative approach that enabled us to obtain locally optimal solutions of this nonconvex design problem in polynomial time. Although potentially suboptimal, for some network scenarios, the data routes and power allocations obtained by our technique enabled achieving significantly higher rates than those achieved by their optimal counterparts in scheduling-based cross layer designs. 


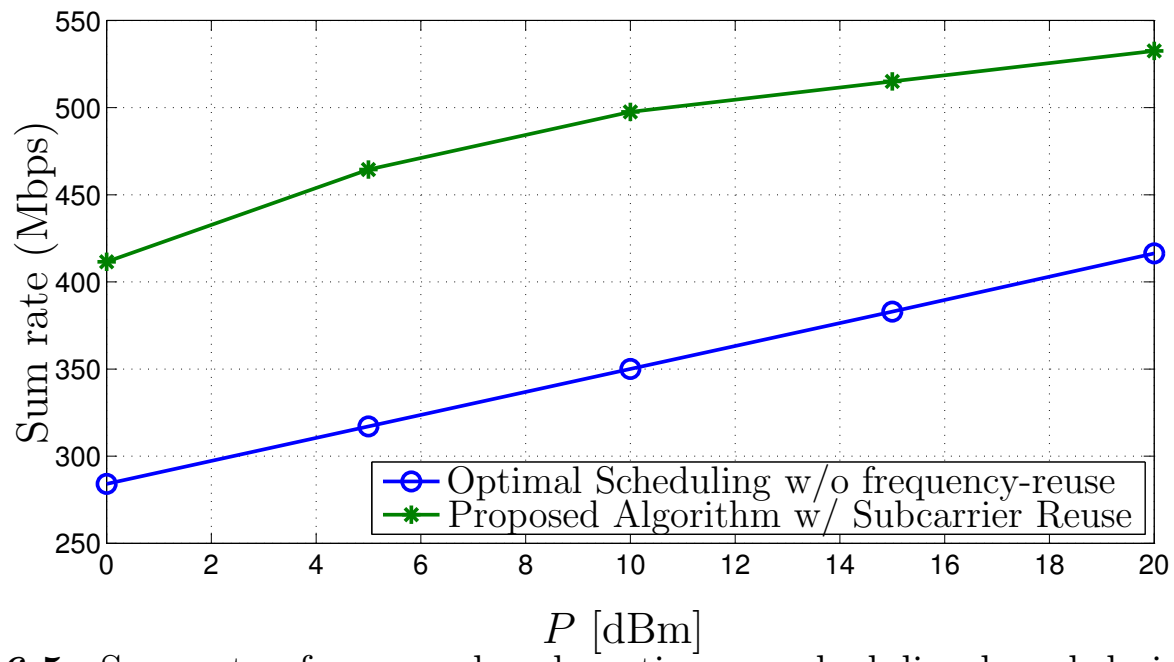

Figure 6.5: Sum rate of proposed and continuous scheduling-based designs for the network considered in Example 3. 


\section{Chapter 7}

\section{JRSPA with Time-Sharing and with Frequency-Reuse}

\subsection{Introduction}

In this chapter, we consider the JRSPA problem when each subcarrier can be both reused and time-shared by multiple links. In this case, the joint design problem incorporates continuous subcarrier scheduling, in addition to data routing and power allocation. As such, this case generalizes the case considered in the previous section and the cases in which frequency-reuse is not considered, in Chapters 4 and 5 . We will first provide mathematical formulations for the constraints that must be satisfied by the design variables and then we will provide a computationally-efficient GP-based approximation method for obtaining locally optimal solutions.

\subsection{System Constraints}

In this section, we derive the mathematical constraints that must be satisfied by any feasible set of data routes, power allocations and time-sharing schedules.

\subsubsection{Routing Constraints}

The constraints that must be satisfied by the routing variables, $\left\{x_{\ell k}^{(d)}\right\}$, are the same as those described in (4.1)-(4.3). 


\subsubsection{Scheduling Constraints}

Considering both time-sharing and frequency-reuse requires us to introduce a new set of variables to refer to the links that operate simultaneously on the same subcarrier for a fraction of the signalling interval. To do so, we let $\gamma_{\ell_{1} \ell_{2} \ldots \ell_{m}}^{(k)}$ be the scheduling variable that determines the fraction of the signalling interval during which links $\ell_{1}, \ldots, \ell_{m} \in \mathcal{L}$ are simultaneously 'active' on subcarrier $k \in \mathcal{K}$ and the remaining $L-m$ links in $\mathcal{L}$ are 'silent' on this subcarrier. Without loss of generality, we will write the indices in an ascending order, i.e., $\ell_{1}<\ell_{2}<\cdots<\ell_{m}$. For notational convenience, let $\Gamma$ be the set containing all the subcarrier time-sharing schedules described above. The cardinality of $\Gamma$ is given by $|\Gamma|=K \sum_{i=1}^{L}\left(\begin{array}{c}L \\ i\end{array}\right)=K\left(2^{L}-1\right)$, where the first consecutive combination in the summation argument represents the number of variables accounted for one active link per subcarrier, the second combination represents the number of variables accounted for two active links per subcarrier and so on. This characterization extracts the potential of reusing subcarriers in a given network, but at the expense of high complexity. As an example, consider a network with $L=3$ links and $K=1$ subcarrier. The set $\Gamma$ has 7 elements, which can be written as $\Gamma=\left\{\gamma_{1}^{(1)}, \gamma_{2}^{(1)}, \gamma_{3}^{(1)}, \gamma_{1,2}^{(1)}, \gamma_{1,3}^{(1)}, \gamma_{2,3}^{(1)}, \gamma_{1,2,3}^{(1)}\right\}$. Suppose that we have the scheduling table in Figure 7.1. For this scheduling, $\gamma_{1}^{(1)}=0.5, \gamma_{1,2}^{(1)}=0.2, \gamma_{1,2,3}^{(1)}=0.3$, and all the other elements in $\Gamma$ are zero.

Signalling interval for $k=1$.

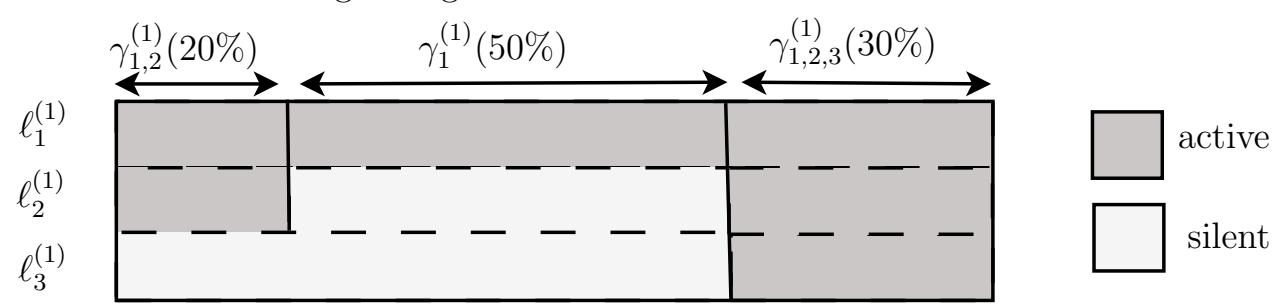

Figure 7.1: An exemplary scheduling for a network with $L=3, K=1$.

The fact that the channels are assumed constant over the signalling interval implies that only the time-sharing schedules (i.e., the values of the entries of $\Gamma$ ) affect the rate expressions, irrespective of the particular time interval over which the subcarriers are time-shared. In other words, permuting the shaded blocks in Figure 7.1 horizontally does not affect the rate expressions. For instance, the two cases illustrated in Figure 7.2 have the same value of $\Gamma$ as the previous example. 
Signalling interval for $k=1$.

\begin{tabular}{|c|c|c|c|}
\hline$\ell_{1}^{(1)}$ & \multicolumn{3}{|c|}{$100 \%$} \\
\hline$\ell_{2}^{(1)}$ & $20 \%$ & $50 \%$ & $30 \%$ \\
\hline$\ell_{3}^{(1)}$ & \multicolumn{2}{|r|}{$70 \%$} & $30 \%$ \\
\hline
\end{tabular}

(a)
Signalling interval for $k=1$.

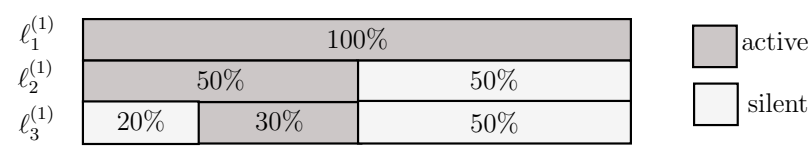

(b)

Figure 7.2: Different ordering of transmissions of the scheduling in Figure 7.1.

The number of variables in $\Gamma$ grows exponentially with the number of links, $L$. This renders the incorporation of $\Gamma$ in the joint optimization computationally prohibitive. In most cases this complexity can be significantly reduced without incurring heavy performance losses. For instance, if the network is tightly coupled, high interference levels render the reuse of subcarriers on multiple links less beneficial. In such a case, restricting the reuse of a subcarrier to a fewer links may incur negligible deterioration in performance but reduces the number of variables significantly. To take advantage of this observation, we limit the number of links that can reuse a particular subcarrier to $I \ll L$. By performing this restriction, the number of elements in $\Gamma$ is reduced from $K\left(2^{L}-1\right)$ to $K \sum_{i=1}^{I}\left(\begin{array}{c}L \\ i\end{array}\right)$, which, for small $I$, is polynomial in $L$. For instance, if at most two links are allowed to reuse a particular subcarrier at any given time, i.e., $I=2$, the number of elements in $\Gamma$ reduces to $\frac{L(L+1)}{2}$. It is worth noting that limiting the number of simultaneous transmissions, $I$, inherently offers a trade-off between performance and complexity. In particular, as $I$ increases, the available resources are utilized more efficiently. However, our numerical results suggest that in dense networks most of the gain of time-sharing and frequency-reuse is accrued by only considering $I \leq 3$ simultaneous transmissions. This number may be larger in clustered networks.

To have feasible time-sharing schedules, the elements in $\Gamma$ must be non-negative and, to avoid overlapping in time, the total amount of time that subcarrier $k \in \mathcal{K}$ is used must not exceed the length of the signalling interval. These constraints imply that

$$
\begin{array}{lr}
\Gamma \geq 0, & \text { elementwise }, \\
\sum_{m=1}^{L} \sum_{\ell_{1} \cdots \ell_{m} \in \mathcal{L}} \gamma_{\ell_{1} \ldots \ell_{m}}^{(k)} \leq 1, & \forall k \in \mathcal{K} .
\end{array}
$$

In writing (7.2), we note that the number of summations depends on $m$, i.e., the 
number of links time-sharing the same subcarrier. For instance, when $m=1$, the argument of the first summation is $\sum_{\ell_{1} \in \mathcal{L}} \gamma_{\ell_{1}}^{(k)}$ and, when $m=2$, it is $\sum_{\ell_{1} \in \mathcal{L}} \sum_{\ell_{2} \in \mathcal{L}} \gamma_{\ell_{1} \ell_{2}}^{(k)}$.

In order to enforce the half-duplex constraint, we must ensure that the nodes do not transmit and receive data on one subcarrier at the same time. In other words, the half-duplex requirement imply that any two links, $\ell_{1} \in \mathcal{L}_{-}(n)$ and $\ell_{2} \in \mathcal{L}_{+}(n)$ (i.e., $a_{n \ell}^{+} a_{n \ell^{\prime}}^{-}=1$ ), cannot be active on the same subcarrier $k \in \mathcal{K}$ at the same time. This further implies that all the time-sharing schedules that correspond to $\ell_{1}$ and $\ell_{2}$, i.e., $\gamma_{\ell_{1} \ldots \ell_{m}}^{(k)}, m=2, \ldots, L$, must be zero. Since all the entries in $\Gamma$ are non-negative, this constraints can be written as

$$
a_{n \ell_{1}}^{+} a_{n \ell_{2}}^{-}\left(\gamma_{\ell_{1} \ell_{2}}^{(k)}+\sum_{m=3}^{L} \sum_{\ell_{3} \ldots \ell_{m} \in \mathcal{L}} \gamma_{\ell_{1} \ldots \ell_{m}}^{(k)}\right)=0, \quad \ell_{1} \in \mathcal{L}, \ell_{2} \in \mathcal{L} \backslash\left\{\ell_{1}\right\}, k \in \mathcal{K}
$$

where $a_{n \ell}^{+}=\max \left\{0, a_{n \ell}\right\}$ and $a_{n \ell}^{-}=\left|\min \left\{0, a_{n \ell}\right\}\right|$, cf. Section 6.2.2.

Similar to the constraint in (6.1), the nodes cannot broadcast data to different destinations at the same time. More specifically, at any time instant, node $n$ can have at most one active link on subcarrier $k$. Hence, all the time-sharing schedules that correspond to more than one outgoing link of node $n$ must be zero. This can be represented as

$$
a_{n \ell_{1}}^{+} a_{n \ell_{2}}^{+}\left(\gamma_{\ell_{1} \ell_{2}}^{(k)}+\sum_{m=3}^{L} \sum_{\ell_{3} \cdots \ell_{m} \in \mathcal{L}} \gamma_{\ell_{1} \ldots \ell_{m}}^{(k)}\right)=0, \quad \ell_{1} \in \mathcal{L}, \ell_{2} \in \mathcal{L} \backslash\left\{\ell_{1}\right\}, k \in \mathcal{K}
$$

Note that (7.3) and (7.4) are trivial when $a_{n \ell}^{+} a_{n \ell^{\prime}}^{-}=0$ and $a_{n \ell}^{+} a_{n \ell^{\prime}}^{+}=0$, respectively.

\subsubsection{Power Allocation Constraints}

In characterizing the power allocation constraints, we denote the power allocated for transmission on subcarrier $k$ of link $\ell$ by the variables $\left\{q_{\ell k}\right\}$. This variable must satisfy the non-negativity constraints in (6.4) and the power budget constraint. In writing the latter constraint, we note that only the subcarriers scheduled to outgoing links contribute to the power consumption of each node. More specifically, if $\ell_{1} \in$ $\mathcal{L}_{+}(n)$, then all the time-sharing schedules that correspond to $\ell_{1}$ contribute to the 
power consumption at node $n$. This constraint can be written as

$$
\sum_{k \in \mathcal{K}} \sum_{\ell_{1} \in \mathcal{L}_{+}(n)} q_{\ell_{1} k}\left(\gamma_{\ell_{1}}^{(k)}+\sum_{m=2}^{L} \sum_{\ell_{2} \cdots \ell_{m} \in \mathcal{L}} \gamma_{\ell_{1} \ldots \ell_{m}}^{(k)}\right) \leq P_{n}, \quad n \in \mathcal{N}
$$

\subsubsection{Capacity Constraints}

To characterize the capacity constraints, we note that the transmission on link $\ell \in \mathcal{L}$ and subcarrier $k \in \mathcal{K}$ is composed of two parts. The first part accounts for the fraction of time over which this transmission is interference-free, whereas the second part accounts for the fraction of time over which this transmission interferes with other transmissions. To characterize the second part, we identify the interfering links and the fraction of time over which these links are interfering. To do so, we note that, if subcarrier $k$ is time-shared by links $\ell_{1}, \ldots, \ell_{m}$, then the transmission on links $\ell_{2}, \ldots, \ell_{m}$ interfere with the transmission on link $\ell_{1}$. Hence, the SINR expression for the transmission on link $\ell_{1}$ is $\frac{q_{\ell_{1} k} g_{\ell_{1} k}}{1+\sum_{i=2}^{m} q_{\ell_{i} k} g_{\ell_{i}^{\prime} k}}$, where $\ell_{i}^{\prime}$ denotes the index of the link connecting the node at which link $\ell_{i}$ originates to the node at which link $\ell_{1}$ ends. Since links $\ell_{1}, \ldots, \ell_{m}$ are simultaneously active on subcarrier $k$ for a fraction of $\gamma_{\ell_{1} \ldots \ell_{m}}^{(k)}$, the expression for the data rate that can be communicated over link $\ell_{1}$ is $\gamma_{\ell_{1} \ldots \ell_{m}}^{(k)} \log \left(1+\frac{q_{\ell_{1} k} g_{\ell_{1} k}}{1+\sum_{i=2}^{m} q_{\ell_{i} k} g_{\ell_{i}^{\prime} k}}\right)$. Summing over all possible combinations of the interfering links, the capacity constraint on the aggregate flow of link $\ell_{1}$ on subcarrier $k$ can be expressed as

$$
\sum_{d \in \mathcal{D}} x_{\ell_{1} k}^{(d)} \leq \gamma_{\ell_{1}}^{(k)} \log \left(1+q_{\ell_{1} k} g_{\ell_{1} k}\right)+\sum_{m=2}^{L} \sum_{\ell_{2} \ldots \ell_{m} \in \mathcal{L}} \gamma_{\ell_{1} \ldots \ell_{m}}^{(k)} \log \left(1+\frac{q_{\ell_{1} k} g_{\ell_{1} k}}{1+\sum_{i=2}^{m} q_{\ell_{i} k} g_{\ell_{i}^{\prime} k}}\right) .
$$

\subsection{Problem Formulation}

Combining the objective described in Section 3.6 with the constraints described in Section 7.2, yields the following formulation:

$$
\begin{aligned}
\max _{\left\{s_{n}^{(d)}\right\},\left\{x_{\ell k}^{(d)}\right\},\left\{q_{\ell k}\right\}, \Gamma} & \sum_{d \in \mathcal{D}} \sum_{n \in \mathcal{N} \backslash\{d\}} w_{n}^{(d)} s_{n}^{(d)}, \\
\text { subject to } & \text { Routing constraints in (4.1)-(4.3), }
\end{aligned}
$$


Scheduling constraints in (7.1)-(7.4),

Power allocation constraints in (6.4) and (7.5),

Capacity constraints in (7.6).

The optimization problem in (7.7) is highly nonconvex because of the scheduling constraints in (7.3) and (7.4), the power allocation constraint in (7.5) and the capacity constraints in (7.6). In particular, although a convex combination of two time-sharing schedules results in a feasible one, this problem does not result in a convex problem. The reason is that power allocations may not necessarily be combined the same as schedules and hence, the problem is not convex in both powers and schedules. To alleviate this difficulty, in the next section, we will develop a GP-based algorithm, analogous to the one described in Section 6.4, to obtain a locally optimal solution for this problem.

\subsection{Proposed GP-based Algorithm}

The optimization problem in (7.7), although nonconvex, is amenable to the GPbased monomial approximation described in Appendix A.2. To use this approximation, we use (5.9) and (5.10) to transform $\left\{s_{n}^{(d)}\right\}$ and $\left\{x_{\ell k}^{(d)}\right\}$ to $\left\{t_{n}^{(d)}\right\}$ and $\left\{r_{\ell k}^{(d)}\right\}$, respectively. Using these new variables, the routing constraints are readily expressed in GP-compatible form as described in (6.8b)-(6.8d).

The non-negativity constraints in (6.4) and (7.1) are inherently satisfied in the GP framework. The constraints in (7.2) and (7.5) are already in a GP-compatible form. Analogous to the case considered in Section 6.4, (7.3) and (7.4) are replaced with the following GP-compatible inequality constraints:

$$
\begin{aligned}
& a_{n \ell_{1}}^{+} a_{n \ell_{2}}^{+}\left(\gamma_{\ell_{1} \ell_{2}}^{(k)}+\sum_{m=3}^{L} \sum_{\ell_{3} \cdots \ell_{m} \in \mathcal{L}} \gamma_{\ell_{1} \ldots \ell_{m}}^{(k)}\right) \leq \epsilon, \\
& a_{n \ell_{1}}^{+} a_{n \ell_{2}}^{-}\left(\gamma_{\ell_{1} \ell_{2}}^{(k)}+\sum_{m=3}^{L} \sum_{\ell_{3} \cdots \ell_{m} \in \mathcal{L}} \gamma_{\ell_{1} \ldots \ell_{m}}^{(k)}\right) \leq \epsilon,
\end{aligned}
$$

where $\epsilon$ is an arbitrary small positive number.

Now, the only remaining constraints that are not GP-compatible are those in (7.6). Invoking the change of variables in (5.10), we can rewrite this set of constraints as 
follows:

$$
\prod_{d \in \mathcal{D}} r_{\ell_{1} k}^{(d)} \leq\left(1+q_{\ell_{1} k} g_{\ell_{1} k}\right)^{\gamma_{\ell_{1}}^{(k)}} \times \prod_{m=2}^{L} \prod_{\ell_{2} \ldots \ell_{m} \in \mathcal{L}}\left(1+\frac{q_{\ell_{1} k} g_{\ell_{1} k}}{1+\sum_{i=2}^{m} q_{\ell_{i} k} g_{\ell_{i}^{\prime} k}}\right)^{\gamma_{\ell_{1} \ldots \ell_{m}}^{(k)}}, \ell_{1} \in \mathcal{L}, k \in \mathcal{K} .
$$

Although the RHS of (7.9) is not a monomial, it is amenable to the monomial approximation technique described in Appendix A.2 [9]. To use this technique, one can approximate all the terms in the RHS of (7.9) by one monomial. However, this approach is overly complicated, and an alternative is to approximate each term in the RHS of (7.9) by a monomial. Noting that the product of monomials is another monomial, a monomial approximation of the RHS of (7.9) is given by

$$
\prod_{d \in \mathcal{D}} r_{\ell_{1} k}^{(d)} \leq \mathcal{M}\left(\left(1+q_{\ell_{1} k} g_{\ell_{1} k}\right)^{\gamma_{\ell_{1}}^{(k)}}\right) \times \prod_{m=2}^{L} \prod_{\ell_{2} \cdots \ell_{m} \in \mathcal{L}} \mathcal{M}\left(\left(1+\frac{q_{\ell_{1} k} g_{\ell_{1} k}}{1+\sum_{i=2}^{m} q_{\ell_{i} k} g_{\ell_{i}^{\prime} k}}\right)^{\gamma_{\ell_{1} \ldots \ell_{m}}^{(k)}}\right)
$$

where the functional $\mathcal{M}(\cdot)$ is described in Appendix A.2. Note that, since $\left\{\gamma_{\ell_{1} \ell 2 \ldots \ell_{m}}^{(k)}\right\}$ are variables, they are inseparable from the argument of $\mathcal{M}(\cdot)$.

Now, the problem in (7.7) can be approximated by the following GP:

$$
\begin{aligned}
\max _{\left\{t_{n}^{(d)}\right\},\left\{r_{\ell k}^{(d)}\right\},\left\{q_{\ell k}\right\}, \Gamma} & \prod_{d \in \mathcal{D}} \prod_{n \in \mathcal{N} \backslash\{d\}}\left(t_{n}^{(d)}\right)^{w_{n}^{(d)}}, \\
\text { subject to } & \text { Routing constraints in }(6.8 \mathrm{~b})-(6.8 \mathrm{~d}), \\
& \text { Power allocation constraints in (7.5), } \\
& \text { Scheduling constraints in (7.2) and (7.8), } \\
& \text { Approx. capacity constraints in (7.10). }
\end{aligned}
$$

Solving this problem iteratively, similar to the algorithm explained in Section 6.4, is guaranteed to yield to a locally optimal solution of the joint design problem in (7.7). More specifically, starting from a feasible point, $\left(\left\{q_{\ell k}^{(0)}\right\}, \Gamma^{(0)}\right)$, we solve the GP problem in (8.4). The solution is then used as an initial point for the next iteration.

A summary of this algorithm is described in Table 7.1 . 
Table 7.1: Successive GP-based Algorithm for Solving (8.4)

1- Let $U^{(0)}=0$. Set accuracy, $\delta>0$, and error tolerance, $\epsilon \geq 0$.

2- Choose $I \in\{1, \ldots, L\}$ and a feasible $\left(\left\{q_{\ell k}^{(0)}\right\}, \Gamma^{(0)}\right)$.

3- Solve the GP in (7.7). Denote the value of the objective by $U$.

4- While $U-U^{(0)} \geq \delta$,

$$
\begin{aligned}
& \left\{q_{\ell k}^{(0)}\right\} \leftarrow\left\{q_{\ell k}\right\}, \\
& U^{(0)} \leftarrow U,
\end{aligned}
$$

Solve the GP in (7.7). Denote the value of the objective by $U$,

\section{End.}

5- Remove the elements in $\Gamma$ that are less than $\epsilon$.

6- Use (5.10) to recover $\left\{s_{n}^{(d)}\right\}$ and $\left\{x_{\ell k}^{(d)}\right\}$.

\subsection{Complexity Analysis}

In this section we examine the computational complexity required for solving the JRSPA problem with both time-sharing and frequency-reuse. The algorithms in Section 7.4, solves the families of the optimization problems in (8.4), iteratively. Each of these problems is in the form of a GP that can be readily converted to a convex optimization problem and can be efficiently solved using IPM-based solvers.

Unfortunately, the log-barrier functions related to the problem in (8.4) is not selfconcordant. Analogous to the discussions in Sections 5.5 and 6.5, we introduce a set of auxiliary variables and constraints in Appendix C.5 which, although redundant, enable us to construct a self-concordant log-barrier function. Using this function and the results in [8], we have the following proposition for the computational complexity of solving the problem in (8.4).

Proposition 4. The complexity of solving (8.4) is of order

$$
\mathcal{O}\left(\left(2 L K N+N+D(N-1)+2 K\left(2^{L}-1\right)\right)^{3.5}\right)
$$

Proof. See Appendix C.5.

Proposition 4 shows that the complexity of solving the problem in (8.4) grows 
exponentially with the number of links, $L$. In particular, it grows as $2^{3.5 L}$. This complexity arises from the fact that the number of elements in $\Gamma$ grows exponentially with $L$, i.e.,

$$
|\Gamma|=K\left(\left(\begin{array}{l}
L \\
1
\end{array}\right)+\left(\begin{array}{l}
L \\
2
\end{array}\right)+\cdots+\left(\begin{array}{l}
L \\
L
\end{array}\right)\right)=K\left(2^{L}-1\right)
$$

Hence, in its general form, this algorithm is suitable for designing small-to-medium size networks. For larger networks, this algorithm can be readily simplified by limiting the number of links that can time-share a particular subcarrier. For instance, if, at most, two links are allowed to time-share one available subcarrier, then the number of elements in $\Gamma$ will be $\left(\begin{array}{l}L \\ 1\end{array}\right)+\left(\begin{array}{l}L \\ 2\end{array}\right)$ which decreases the number of variables in $\Gamma$ dramatically and makes the algorithm computationally efficient for larger networks.

\subsection{Numerical Results}

In this section we provide numerical results to evaluate the performance of joint routing and resource allocation algorithms for the case with both time-sharing and frequency-reuse. For this case, we first provide an illustrative example and then we use Monte Carlo simulations to demonstrate the advantage of the designs in which each subcarrier can be used simultaneously by multiple links over those in which each subcarrier can be used by only one link at any given time instant. For our simulations, we use a setup analogous to the one used for the first example of Section 6.6.

Example 1: (Performance Evaluation) Consider an exemplary network with $N=4$ nodes. In this network, as before, nodes 3 and 4 wish to communicate with nodes 2 and 1 , respectively, over $K=2$ subcarriers. In particular, for destination node $d=1$, node $n=4$ is the source and nodes $\{2,3\}$ are potential relays, and, for destination node $d=2$, node $n=3$ is the source and nodes $\{1,4\}$ are potential relays.

The considered network has $L=12$ directional links and therefore the channel matrix has $12 \times 2$ elements. The normalized channel gain of link $\ell$ on subcarrier $k$ in $\mathrm{dB}$, i.e., $10 \log _{10} g_{\ell k}$, is given in Table 7.2.

In this example, the nodes power budget is set to $P=20 \mathrm{dBm}$ and the two rates, $s_{3}^{(2)}$ and $s_{4}^{(1)}$, are assigned equal weights, i.e., $w_{3}^{(2)}=w_{4}^{(1)}=1$. Since in this example time-sharing is allowed, the algorithm in Section 7.4 is used to generate the data routes, time-sharing schedules and power allocations. The sum-rate yielded by the 
Table 7.2: Channel gains $[\mathrm{dB}]$ in Example 1.

\begin{tabular}{l|ccccccccccccc} 
& link 1 & link 2 & link 3 & link 4 & link 5 & link 6 & link 7 & link 8 & link 9 & link 10 & link 11 & link 12 \\
\hline subcarrier 1 & -6.1 & -11.1 & 1.86 & -12.3 & -13.5 & 42.4 & 15.82 & -2.43 & -5.82 & -6.9 & 35.0 & 3.304 \\
subcarrier 2 & -5.7 & 1.57 & 5.64 & -5.98 & -19.2 & 40.7 & -3.38 & -5.61 & 0.871 & -0.6 & 41.2 & -14.5
\end{tabular}

algorithm in Section 7.4 is $7.4 \mathrm{~b} / \mathrm{s} / \mathrm{Hz}$. The data routes generated by this algorithm are illustrated in Figure 7.3. For ease of exposition, the network in this example is split into the two sub-networks: the one in Figure 7.3(a) depicts the routes of the data intended for destination $d=1$, and the one in Figure 7.3(b) depicts the routes of the data intended for destination $d=2$. The complete network is the superposition of the two sub-networks. For instance, the data transmitted over link 7, connecting node 3 to node 1 , is $4.8 \mathrm{~b} / \mathrm{s} / \mathrm{Hz}$, of which $2 \mathrm{~b} / \mathrm{s} / \mathrm{Hz}$ is intended for destination $d=1$ and $2.8 \mathrm{~b} / \mathrm{s} / \mathrm{Hz}$ is intended for destination $d=2$.

The time-sharing schedules of the subcarriers generated by the algorithm in Section 7.4 are provided in Figure 7.4. It can be seen from this figure that subcarrier $k=1$ is both reused and time-shared, whereas subcarrier $k=2$ is only time-shared. Figures 7.3 and 7.4 imply that link 7 , connecting node 3 to node 1 , and link 11, connecting node 4 to node 2 , carry the data intended for both destinations on the same subcarrier, $k=1$, during the same time interval. The fact that our designs enforce half-duplex requirement can be inferred from Figures 7.3 and 7.4. For instance, Figure 7.3(a) shows that node 3 uses the same subcarrier, $k=1$, for its transmission and reception on links 7 and 12, respectively, but in different time intervals, as indicated in Figure 7.4.

The power allocations yielded by the algorithm in Section 7.4 are shown in Table 7.3. This table shows that because of frequency-reuse, the nodes do not necessarily use their total power budgets. This is due to the fact that, in this scenario, when a node increases its transmission power, it inflicts high interference on other transmissions. This is in contrast with the situation considered in Chapters 4 and 5, wherein frequency-reuse is not allowed and increasing the transmitted power of a node does not affect the transmissions of the other nodes in the network. 


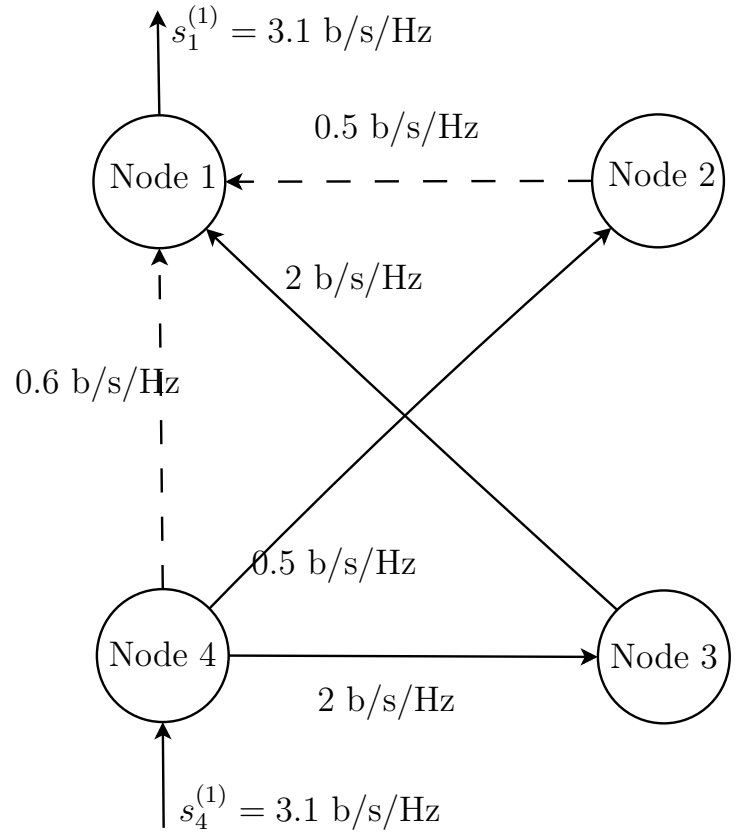

(a)

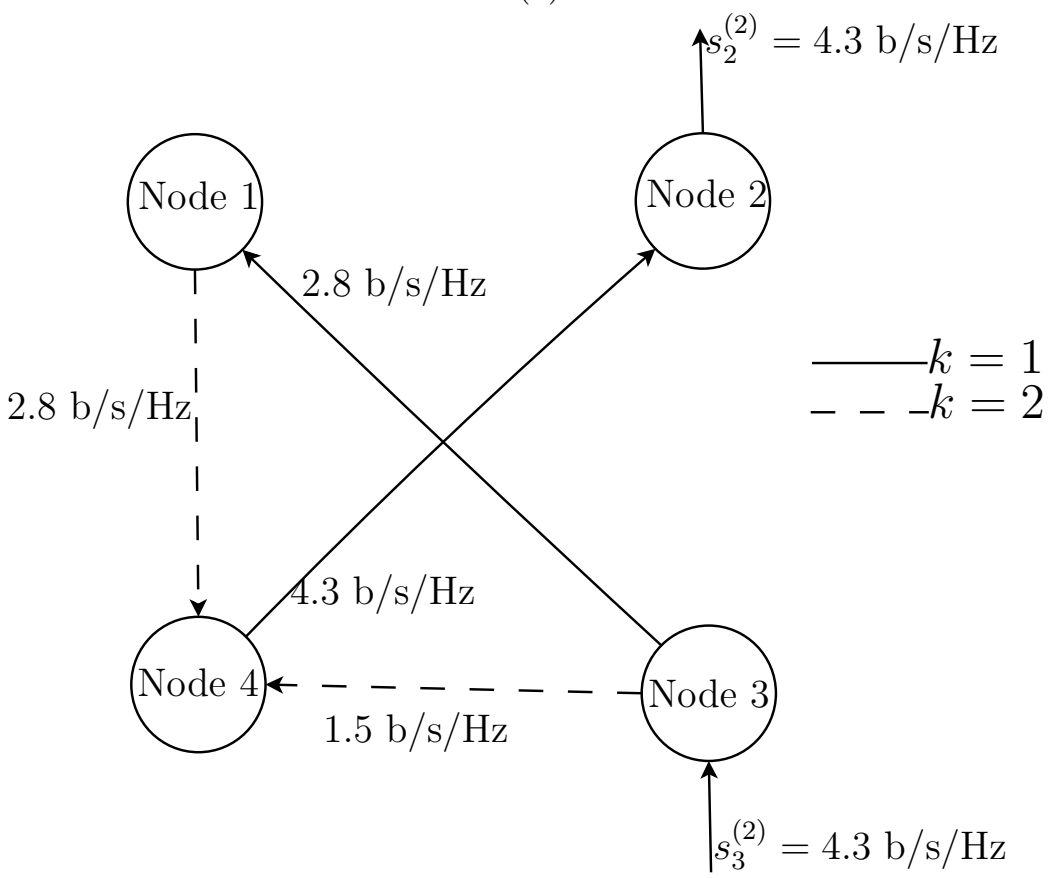

(b)

Figure 7.3: Data routes for (a) $d=1$, (b) $d=2$ in Example 1. 


\begin{tabular}{|c|c|c|c|c|c|c|}
\hline \multicolumn{3}{|c|}{$k=1$} & \multicolumn{4}{|c|}{$k=2$} \\
\hline \multirow{2}{*}{$\begin{array}{r}\text { Link } 7 \\
\text { Link } 11\end{array}$} & $60 \%$ & & \multirow{4}{*}{$\begin{array}{l}\text { Link } 3 \\
\text { Link } 4 \\
\text { Link } 9 \\
\text { Link } 10\end{array}$} & $45 \%$ & & \\
\hline & $60 \%$ & & & & & \\
\hline \multirow[t]{2}{*}{ Link 12} & & $40 \%$ & & & $30 \%$ & \\
\hline & & & & & & $15 \%$ \\
\hline
\end{tabular}

Figure 7.4: Time-sharing schedules of the subcarriers in Example 1.

Table 7.3: Power allocations $(\mathrm{mW})$ in Example 1.

\begin{tabular}{|l|l|l|l|}
\hline$n=1$ & $n=2$ & $n=3$ & $n=4$ \\
\hline$q_{3,2}=23$ & $q_{4,2}=56.5$ & $q_{7,1}=6.5$ & $q_{10,2}=24.5$ \\
& & $q_{9,2}=13.5$ & $q_{11,1}=0.3$ \\
& & & $q_{12,1}=16.5$ \\
\hline
\end{tabular}


Example 2: (Average Weighted-Sum Rate Comparison) In this example, we use Monte Carlo simulations to evaluate the performance of the joint designs with and without time-sharing. We consider a network with $N=4$ nodes in which nodes 3 and 4 wish to communicate with nodes 2 and 1, respectively, over $K=4$ subcarriers. The numerical results are averaged over 10 independent network realizations.

The average weighted-sum rates yielded by the algorithms in Sections 6.4 and 7.4 for the values of $P$ ranging from 0 to $30 \mathrm{dBm}$ are depicted in Figures 7.5(a), 7.5(b) and $7.5(\mathrm{c})$ for the cases of $w_{3}^{(2)}=5 w_{4}^{(1)}, w_{3}^{(2)}=w_{4}^{(1)}$, and, $w_{3}^{(2)}=\frac{1}{5} w_{4}^{(1)}$, respectively. These figures also provide a comparison with the weighted-sum rates yielded by the designs in which frequency-reuse is not considered, cf. Chapters 4 and 5 .

As can be seen from Figure 7.5, the weighted-sum rate yielded by the joint design with both time-sharing and frequency-reuse outperforms the designs in which either time-sharing or frequency-reuse is exclusively considered. For instance, Figure 7.5(b) suggests that, at the sum-rate of $12 \mathrm{~b} / \mathrm{s} / \mathrm{Hz}$, the proposed design with both timesharing and frequency-reuse yields a power advantage of $4 \mathrm{dBm}$ over the designs with either time-sharing or frequency-reuse and a power advantage of $8 \mathrm{dBm}$ over the design without time-sharing and frequency-reuse. This figure also suggests that for values of $P$ less than $15 \mathrm{dBm}$, the design with frequency-reuse but without time-sharing yields better performance than the design with time-sharing but without frequency-reuse. However, for values of $P$ higher than $15 \mathrm{dBm}$, the design with time-sharing only performs better than the one with frequency-reuse only. This phenomenon can be attributed to the effect of interference. At low powers, the effect of interference is small and frequency-reuse performs generally better than time-sharing. In contrast, at high powers, the effect of interference is more severe and time-sharing performs generally better than frequency-reuse. As expected, the design with neither timesharing nor frequency-reuse has inferior performance.

Example 3: (Generalized JRSPA with Reduced Simultaneous Transmissions) In this example, we evaluate the performance of the algorithm developed in Section 7.4. We consider a snapshot of a network with $N=5$ nodes and $L=20$ links (links with a distance more than $150 \mathrm{~m}$ are neglected). In this network nodes 3 and 4 wish to communicate with nodes 2 and 1 , respectively, over $K=4$ subcarriers. The number of simultaneous transmissions is set to $I=20, I=3$ and $I=2$, which results in $\Gamma$ with $2^{20}-1,190$ and 110 variables, respectively. 


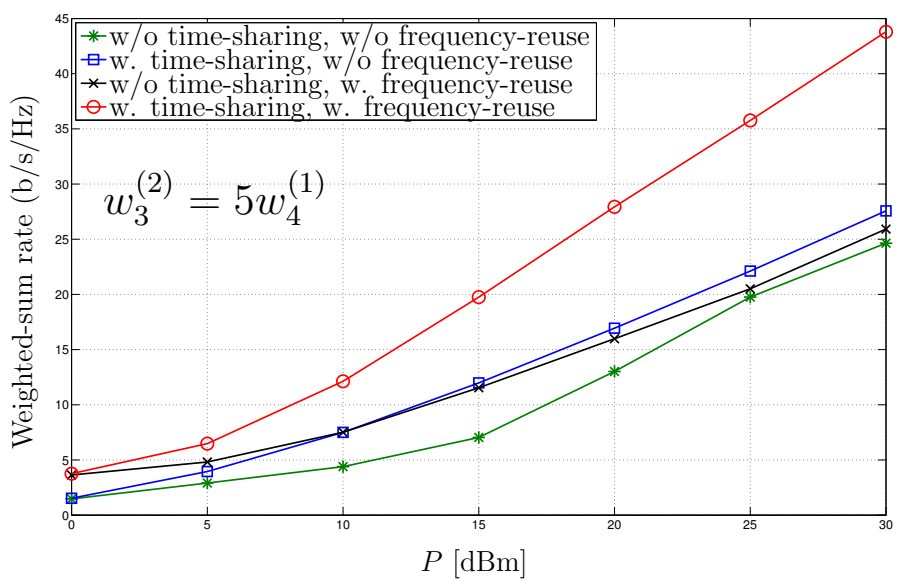

(a)

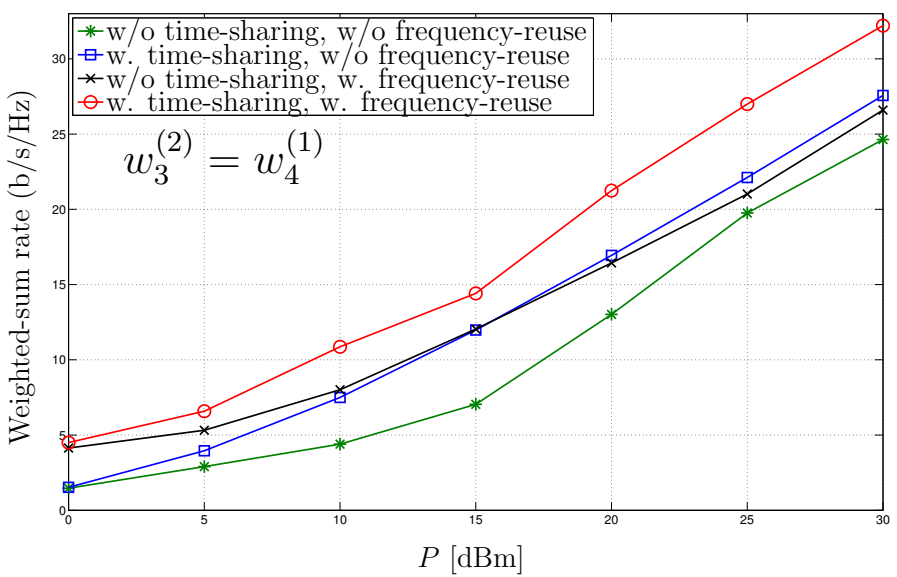

(b)

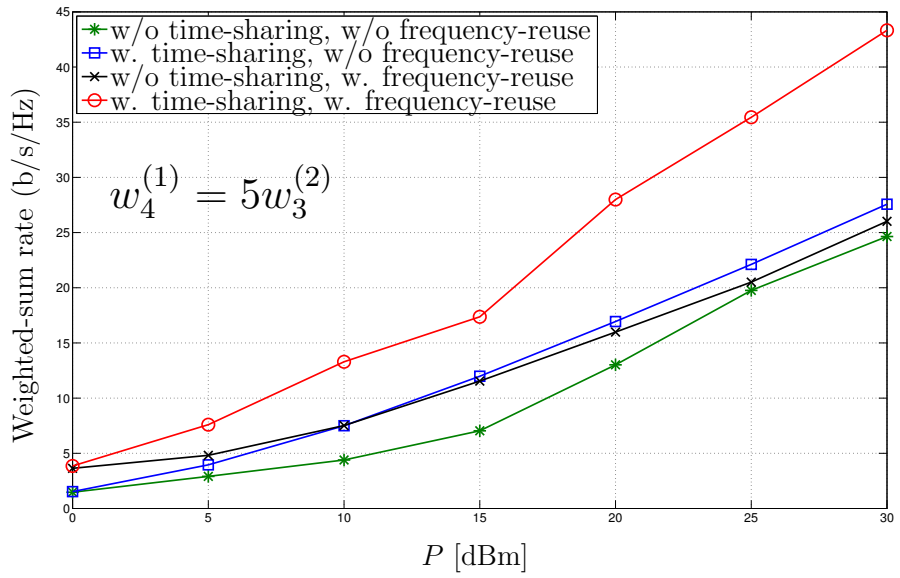

(c)

Figure 7.5: Average weighted-sum rate comparison for (a) $w_{3}^{(2)}=5 w_{4}^{(1)}$, (b) $w_{3}^{(2)}=$ $w_{4}^{(1)}$, and $(\mathrm{c}) w_{4}^{(1)}=5 w_{3}^{(2)}$. 
The sum-rate yielded by the generalized algorithm with different values of $I$ is depicted in Figure 7.6. For comparison, this figure also shows the rates yielded by the special case in Chapter 6. As can be seen from Figure 7.6, the algorithm with $I=2$ and 3 yields rates that are slightly less than the rate yielded by the algorithm with $I=L$, however with a significantly less computational complexity. In fact, the complexity of the algorithm with $I \ll L$ is polynomial, whereas that of the algorithm with $I=L$ is exponential in $L$. This feature renders the algorithm with $I \leq 3$ more attractive for designing large networks with potentially rapid channel variations. From Figure 7.6 it can be seen that the gap between the rates yielded by different values of $I$ decreases as the power budget increases. This is because as power increases, interference becomes more severe, which causes the reuse of a particular subcarrier on multiple links less beneficial. It can be also seen from this figure that most of the gain of frequency-reuse is mustered by only considering two or three simultaneous transmissions, i.e., $I \leq 3$. This implies that increasing $I$ trades complexity for performance. In particular, as $I$ increases, the performance of the algorithm becomes closer to that of the one with $I=L$, but at the expense of increased complexity.

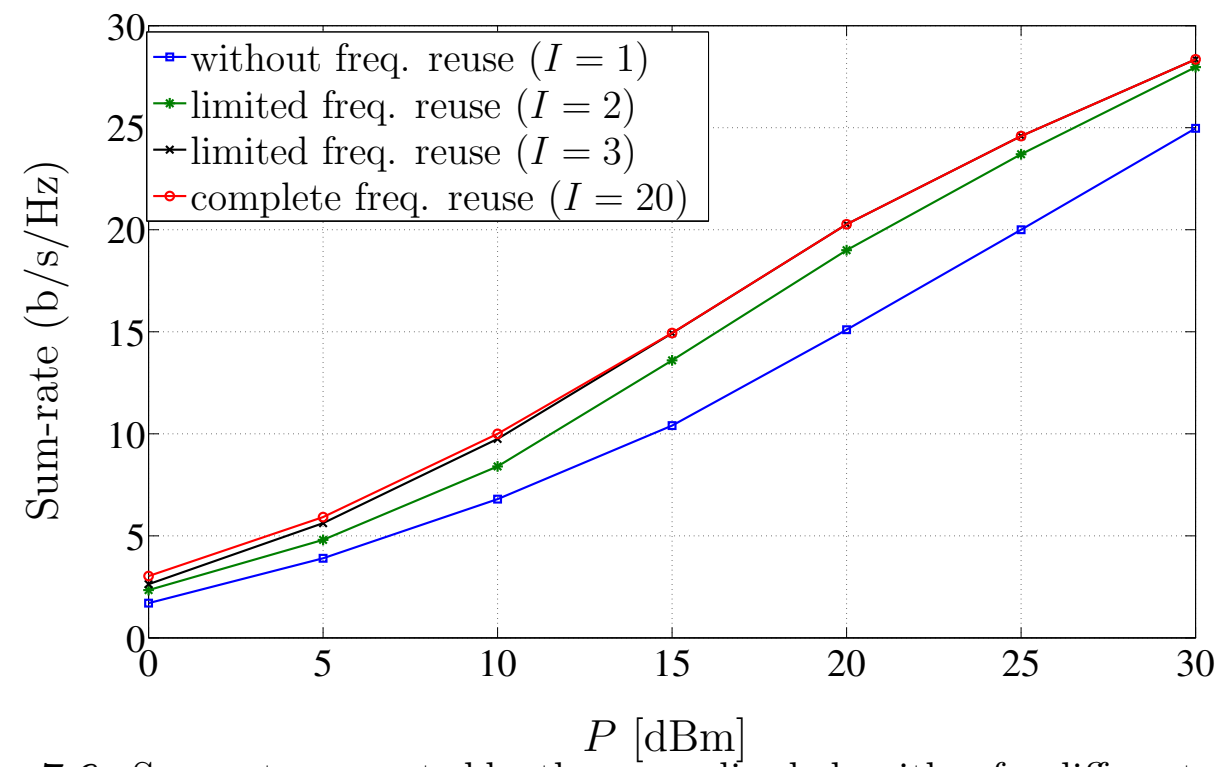

Figure 7.6: Sum-rate generated by the generalized algorithm for different values of $I$.

In Figure 7.7(a) we investigate the convergence behaviour of the generalized algorithm. We consider an instance of a network in which the power budget of all nodes 
is set to $P=10 \mathrm{dBm}$. It can be seen from this figure that, in addition to being significantly less computationally demanding, the algorithm with a lower value of $I$ exhibits considerably faster convergence than that of the one with a higher value of I. This convergence can be further ameliorated by choosing the initial point more carefully, for instance, by choosing this point to be the solution yielded by algorithm in Chapter 4 for the case with no frequency-reuse.

To illustrate the effect of random initialization of the generalized algorithm, in Figure 7.7(b) the value of the objective to which the generalized algorithm with $I=2$ converged is shown for 80 random instances of feasible initial points, $\left(q_{\ell k}^{(0)}, \Gamma^{(0)}\right) \in$ $[0, P]^{L K} \times[0,1]^{|\Gamma|}$. It can be seen from this figure that although the algorithm is relatively sensitive to the choice of the initial point, finding initial points that result in 'good' local maxima is generally easy.

Example 4: (Average Rate-Region Comparison) In this example we provide the rate regions that can be achieved by the algorithms in Section 7.4, when $P=10$ $\mathrm{dBm}$. This region is obtained by varying the weights $\left(s_{4}^{(1)}, s_{3}^{(2)}\right)$ over the unit simplex, i.e., $\left\{\left(w_{4}^{(1)}, w_{3}^{(2)}\right) \mid w_{4}^{(1)} \geq 0, w_{3}^{(2)} \geq 0, w_{4}^{(1)}+w_{3}^{(2)}=1\right\}$, and is depicted in Figure 7.8. Comparisons between this rate region and the ones corresponding to the cases when frequency-reuse or time-sharing is not considered in Chapters 4 and 6 are also provided in this figure. As can be seen from Figure 7.8, the rate region corresponding to the design with both time-sharing and frequency-reuse properly contains the rate regions corresponding to the designs in which either time-sharing or frequency-reuse is exclusively used. It can be also seen that restricting the number of simultaneous transmissions to be less than three suffices to achieve most of the frequency-reuse gain with less computational complexity.

Setting $P=10 \mathrm{dBm}$, in Figure 7.9 the weighted-sum rate achieved by the proposed design in Section 7.4 is depicted for values of $w_{4}^{(1)}=1-w_{3}^{(2)}$ ranging from 0 to 1 . This rate is compared with the ones achieved by the designs in Chapters 4, 5 and 6 . For all sets of weights, the design with both time-sharing and frequency-reuse outperforms the ones with either time-sharing or frequency-reuse. 


\subsection{Conclusion}

In this chapter, we developed a framework for optimizing data routes, subcarrier schedules and power allocations in generic half-duplex interference-limited multicarrier networks. This framework subsumes designs in which time-sharing and frequencyreuse are considered separately. Considering both time-sharing and frequency-reuse jointly gives rise to generally difficult to solve nonconvex optimization problems. To circumvent this difficulty, we invoked an approximation technique based on GP and provided a computationally-efficient method for obtaining locally optimal solutions. Despite its potential performance gains, the proposed optimization framework is centralized and is thus only suitable for designing small-to-medium size networks. For larger networks, this framework can be seen as a benchmark and a first step towards developing effective distributed designs that make judicious use of the degrees of design freedom offered by the physical wireless medium. 


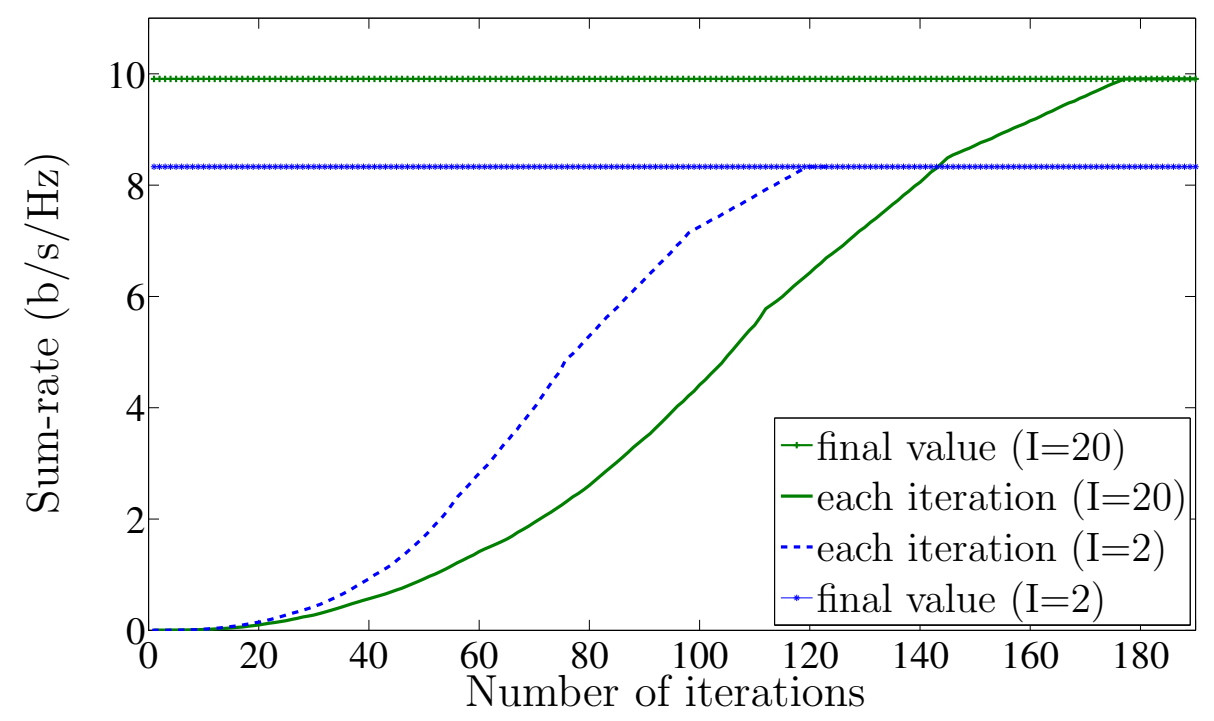

(a)

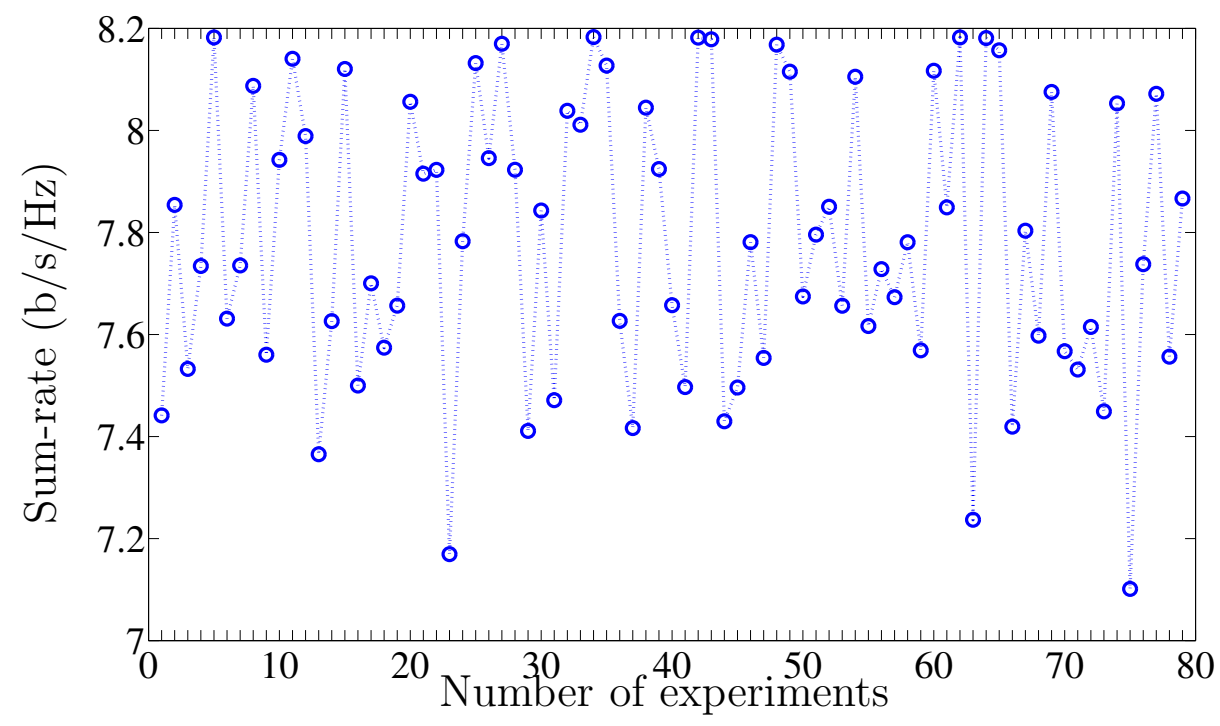

(b)

Figure 7.7: (a) Convergence behaviour and (b) performance of the generalized algorithm with different initial points. 


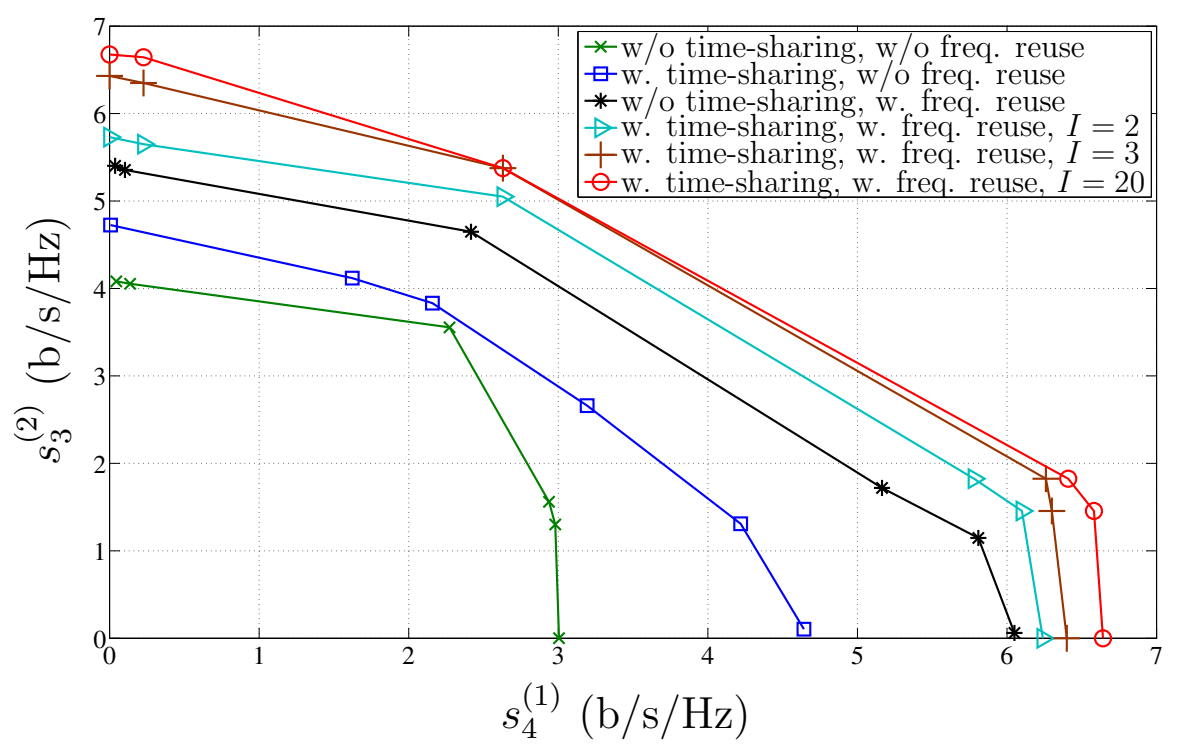

Figure 7.8: Rate-region comparison.

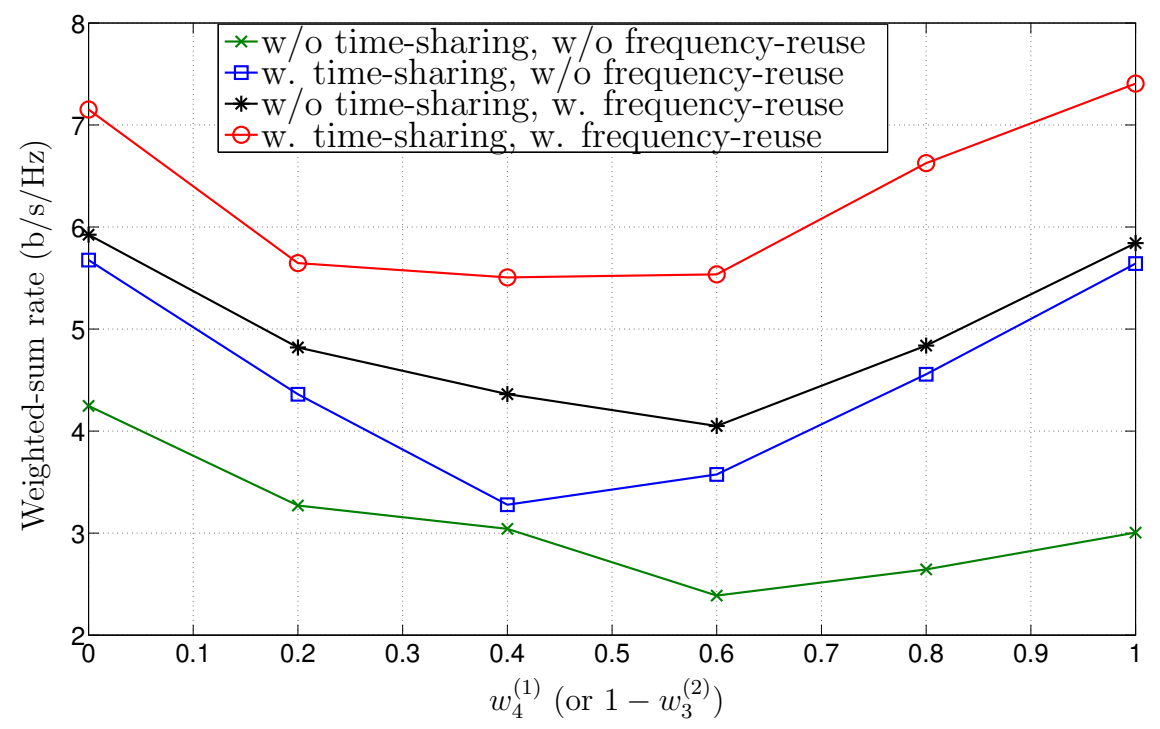

Figure 7.9: Average weighted-sum rate comparison. 


\section{Chapter 8}

\section{Generalized JRSPA: A Low-Complexity Approach}

\subsection{Introduction}

In this chapter, we consider the generalized JRSPA discussed in Chapter 7. The approach that we took there to solve this problem was based on GP and monomial approximations. This approach solved the problem but at the expense of exponential complexity and slow convergence which makes it suitable for designing small size networks. Our goal in this chapter is to propose a low-complexity algorithm that suits implementation for medium-to-large size networks. For simplicity we assume that there is at most one interferer at any time, i.e., at most two links can use a subcarrier simultaneously. Extending this assumption to $I$ simultaneous transmissions can be readily incorporated in the formulations.

\subsection{Network Analysis}

In the most general case, the number of variables in $\Gamma$ grows exponentially with the number of links, $L$. This renders the incorporation of $\Gamma$ in the joint optimization in (7.7) computationally prohibitive.

The complexity of solving (7.7) can be reduced by combining the broadcasting constraint and the half-duplex constraint in (7.3) and (7.4), respectively. In particular, examining these constraints reveals that they are related to the network topology and do not depend on the channel conditions. The reduction in complexity, at least for small networks, appears to be significant. For instance, for fully connected networks 
with $N=4$ nodes and $L=N(N-1)=12$ links, $|\Gamma|$ is reduced from 4095 to 40 .

To characterize this fact, we use $\Gamma_{L \times L}^{(k)}$ to denote the subcarrier schedules on subcarrier $k \in \mathcal{K}$ and $\Gamma$ to denote the set of all matrices, i.e., $\Gamma=\left\{\Gamma^{(k)}, k=1, \ldots, K\right\}$. In each matrix, the entry $\gamma_{\ell \ell^{\prime}}^{(k)}$ represents the fraction of signalling interval over which subcarrier $k$ is used on both links $\ell$ and $\ell^{\prime}$. As, by definition, $\gamma_{\ell \ell^{\prime}}^{(k)}=\gamma_{\ell^{\prime} \ell}^{(k)}, \Gamma$ is a symmetric matrix whose diagonal entries represent the fraction of time during which transmissions do not experience interference and off-diagonal entries represent the fraction of time during which simultaneous transmissions interfere with each other.

The half-duplex and broadcasting constraints on subcarrier $k$ enforce some entries of $\Gamma^{(k)}$ to be zero and hence, can be removed from the variable set. For instance, if $\ell$ and $\ell^{\prime}$ are an incoming and outgoing links of node $n$, respectively, then the halfduplex constraint enforces $\gamma_{\ell \ell^{\prime}}^{(k)}=0$ for all $k \in \mathcal{K}$. Hence, these two constraints can be enforced by pruning the set $\Gamma$ prior to solving (7.7). The pruning rule is as follows: For each $\ell$ and $\ell^{\prime} \in \mathcal{L}$, if either $a_{n \ell_{1}}^{+} a_{n \ell_{2}}^{+}=0$ or $a_{n \ell_{1}}^{+} a_{n \ell_{2}}^{-}=0$, the corresponding timeshares in (7.3) and (7.4) are removed from the set $\Gamma$. Unfortunately, we have not been able to obtain a closed form of the cardinality of the resulting $\Gamma$ in general. However, we managed to compute its cardinality when at most two links are active at the same time.

For a fully connected graph where $L=N(N-1)$, the cardinality of $\Gamma$ can be reduced to

$$
\begin{aligned}
|\Gamma| & =K\left(N(N-1)+\left(\begin{array}{c}
N(N-1) \\
2
\end{array}\right)-N\left(\begin{array}{c}
N-1 \\
2
\end{array}\right)-N\left(\begin{array}{c}
N-1 \\
1
\end{array}\right)\left(\begin{array}{c}
N-1 \\
1
\end{array}\right)\right. \\
& \left.+\frac{N(N-1)}{2}\right)=K N(N-1)\left(1+\frac{(N-2)^{2}}{2}\right),
\end{aligned}
$$

where the first term represents the number of diagonal entries of $\Gamma$. The second term accounts for distinct off diagonal entries of $\Gamma$. The third term accounts for the variables that violate the half-duplex constraint. Finally the last two terms represent the number of variables that violate the broadcasting constraint; the last term compensates for the variables that counted twice in the previous term. In particular, for a two-way link the same schedule is counted twice, one per each end node, which needs to be taken away from the total count. An exemplary network of 3 nodes and its corresponding $\Gamma^{(k)}$ matrix is illustrated in Figure 8.1. 


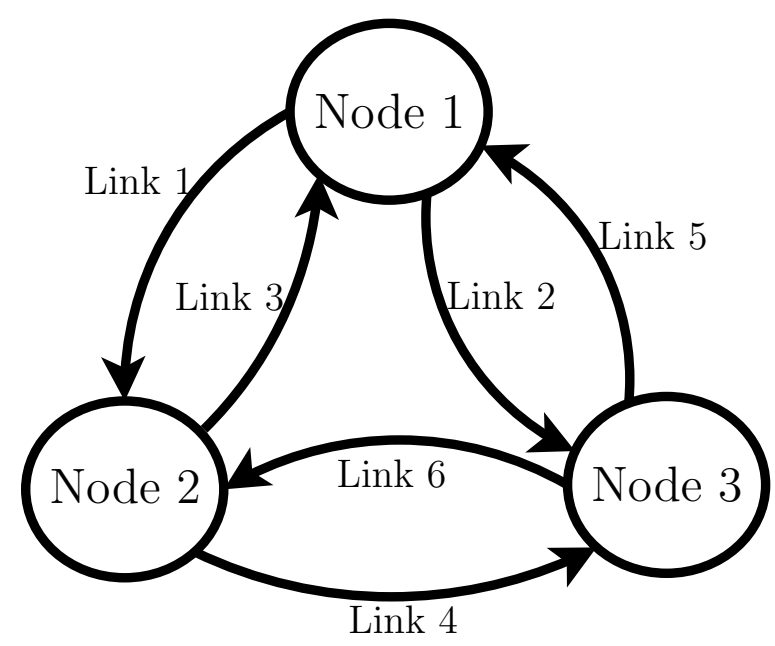

$\Gamma^{(k)}=\left[\begin{array}{cccccc}\gamma_{1,1}^{(k)} & \times & - & - & - & \gamma_{1,6}^{(k)} \\ \times & \gamma_{2,2}^{(k)} & - & \gamma_{2,4}^{(k)} & - & - \\ - & - & \gamma_{3,3}^{(k)} & \times & \gamma_{3,5}^{(k)} & - \\ - & \gamma_{2,4}^{(k)} & \times & \gamma_{4,4}^{(k)} & - & - \\ - & - & \gamma_{3,5}^{(k)} & - & \gamma_{5,5}^{(k)} & \times \\ \gamma_{1,6}^{(k)} & - & - & - & \times & \gamma_{6,6}^{(k)}\end{array}\right], \quad \times$ violates broadcast assumption,

Figure 8.1: The scheduling matrix for a 3-node network with $L=6$ links.

\subsection{Problem Formulation}

In this section we use the formulation derived in (7.7). For simplicity we only assume two simultaneous transmissions. However, the upcoming formulations can be generalized to $I$ simultaneous transmissions.

$$
\max _{\left\{s_{n}^{(d)}\right\},\left\{x_{\ell k}^{(d)}\right\},\left\{q_{\ell k}\right\},\left\{\gamma_{\ell \ell^{\prime}}^{(k)}\right\}} \sum_{d \in \mathcal{D}} \sum_{n \in \mathcal{N} \backslash\{d\}} w_{n}^{(d)} s_{n}^{(d)},
$$

subject to

$$
\begin{array}{ll}
s_{n}^{(d)} \geq 0, & n \in \mathcal{N} \backslash d, d \in \mathcal{D}, \\
x_{\ell k}^{(d)} \geq 0, & \ell \in \mathcal{L}, k \in \mathcal{K}, d \in \mathcal{D}, \\
\sum_{\ell \in \mathcal{L}} \sum_{k \in \mathcal{K}} a_{n \ell} x_{\ell k}^{(d)}=s_{n}^{(d)}, & n \in \mathcal{N} \backslash d, d \in \mathcal{D}, \\
\Gamma \geq 0, & \\
& \text { elementwise, }
\end{array}
$$




$$
\begin{array}{ll}
\sum_{\ell \ell^{\prime} \in \mathcal{L}} \gamma_{\ell \ell^{\prime}}^{(k)} \leq 1, & k \in \mathcal{K}, \\
q_{\ell k} \geq 0, & \ell \in \mathcal{L}, k \in \mathcal{K}, \\
\sum_{k \in \mathcal{K}} \sum_{\ell \in \mathcal{L}+(n)} q_{\ell k} \sum_{\ell^{\prime} \in \mathcal{L}} \gamma_{\ell \ell^{\prime}}^{(k)} \leq P_{n}, & n \in \mathcal{N}, \\
\sum_{d \in \mathcal{D}} x_{\ell k}^{(d)} \leq \gamma_{\ell \ell}^{(k)} \log \left(1+q_{\ell k} g_{\ell \ell}^{(k)}\right) & \\
\quad+\sum_{\ell^{\prime} \in \mathcal{L} \backslash\{\ell\}} \gamma_{\ell \ell^{\prime}}^{(k)} \log \left(1+\frac{q_{\ell k} g_{\ell \ell}^{(k)}}{1+q_{\ell^{\prime} k} g_{\ell \ell^{\prime}}^{(k)}}\right), \ell \in \mathcal{L}, k \in \mathcal{K} .
\end{array}
$$

As stated in Chapter 7, the optimization problem in (8.1) is highly nonconvex because of the constraints in $(8.1 \mathrm{~h})$ and $(8.1 \mathrm{i})$ and hence, generally difficult to solve. An attempt to solve this problem is made in Chapter 7 which was based on GP. The iterative algorithm proposed therein, although finds an approximated solution, has high complexity and generally slow convergence especially for large networks, e.g., networks with more than 10 nodes. To circumvent these difficulties, in this chapter we propose an efficient low-complexity algorithm that exhibits fast convergence even for large networks. Our approach is to decompose the optimization in (8.1) into two smaller sub-problems, one for scheduling and one for power allocation, with a partial coupling between them. These sub-problems will be presented next.

\subsection{Joint Design Sub-problems: Scheduling and Power Allocation}

In this section, we decouple the optimization problem in (8.1) into two parts, one for scheduling when power allocations are fixed and one for power allocations when schedules are fixed. After solving these sub-problems, in Section 8.5 an iterative technique will be developed to obtain a sub-optimal solution of the entire problem in (8.1).

\subsubsection{Scheduling with Fixed Power Allocations}

In this section, we consider the problem of optimizing the subcarrier schedules that maximize a weighted-sum rate of the network when the power allocations are

fixed. Let $\tilde{q}_{\ell k}^{(k)}$ denote the power allocations which are assumed to be fixed in this 
phase. Careful examination of the optimization problem in (8.1) reveals that with the power allocations fixed, this problem becomes an LP and hence, its global maximum can be found in polynomial time. A similar observation has also been made in [48] for a different problem.

\subsubsection{Power Allocation with Fixed Schedules}

In the previous section, we considered the problem of optimizing the subcarrier schedules when the power allocations are fixed. In this section, we consider the complementary problem, i.e., the problem of optimizing the power allocations that maximize a weighted-sum rate of the network when the subcarrier schedules are fixed. Let $\tilde{\Gamma}$ denote the schedules which are assumed to be fixed in this phase. The optimization problem in (8.1) with fixed schedules breaks down into a GP at high SINR regimes. In this case, the solution can be found optimally [11]. However, in general this problem is nonconvex and difficult to solve. In [11], an iterative technique based on monomial approximation was used to find a suboptimal solution. However, in Chapter 7 it was shown that this technique generally has slow convergence, even for small networks. This renders it impractical for medium-to-large networks. In this section, we develop a novel approach to tackle this problem. We will refer to this approach as "constraint-splitting" which will be shown in Section 8.6.3 to have fast convergence.

The first step in developing our approach is to express the capacity constraint in (8.1i) in a form that facilitates the optimization of the power allocations. This constraint can be written in the following form:

$$
\sum_{d \in \mathcal{D}} x_{\ell k}^{(d)}+\underbrace{\sum_{\ell^{\prime} \in \mathcal{L} \backslash\{\ell\}} \gamma_{\ell \ell^{\prime}}^{(k)} \log \left(1+q_{\ell^{\prime} k} g_{\ell \ell^{\prime}}^{(k)}\right)}_{\text {Interference part }} \leq \underbrace{\gamma_{\ell \ell}^{(k)} \log \left(1+q_{\ell k} g_{\ell \ell}^{(k)}\right)+\sum_{\ell^{\prime} \in \mathcal{L}} \gamma_{\ell \ell^{\prime}}^{(k)} \log \left(1+q_{\ell^{\prime} k} g_{\ell \ell^{\prime}}^{(k)}\right)}_{\text {Noisy-signal part }} .
$$

We name the second summation in the RHS of (8.2) as interference part because it has interference only and the summation in the LHS of (8.2) as noisy-signal part because it has both signal and interference.

Looking back into the optimization in (8.1) with fixed schedules and (8.1i) being replaced with (8.2), we make the following observation which will later help us in proposing a fast-convergence technique to solve the power allocation problem. The 
first observation is that, if we fix the noisy-signal part, the optimization problem in (8.1) becomes in a form that is amenable to GP which can be easily converted into a convex problem [9]. The second observation is that, if we fix the interference part, the optimization problem in (8.1) breaks down into a convex form which is efficiently solvable [8]. Taking advantage of theses observations, in the next two sections we will explain each of these convex problems and then we will develop an iterative technique that exhibits fast convergence to a power allocation solution.

\subsubsection{Interference Sub-problem}

In this section we discuss the problem in (8.1) with fixed schedules, $\tilde{\Gamma}$, and with (8.1i) being replaced with (8.2). We consider the case when the RHS of (8.2) is fixed. To fix the RHS, we need an initial power allocation which we denote by $\left\{p_{\ell k}^{(0)}\right\}$. We also introduce a parameter $\alpha \geq 1$ which will be used to control the search region for a proper power allocation around $\left\{p_{\ell k}^{(0)}\right\}$.

Having fixed the RHS of (8.2) and introduced parameter $\alpha$, the joint design problem can be written in the following form:

$$
\max _{\left\{s_{n}^{(d)}\right\},\left\{x_{\ell k}^{(d)}\right\},\left\{q_{\ell k}\right\}} \sum_{d \in \mathcal{D}} \sum_{n \in \mathcal{N} \backslash\{d\}} w_{n}^{(d)} s_{n}^{(d)},
$$

subject to

$$
\begin{array}{ll}
s_{n}^{(d)} \geq 0, & n \in \mathcal{N} \backslash d, d \in \mathcal{D}, \\
x_{\ell k}^{(d)} \geq 0, & \ell \in \mathcal{L}, k \in \mathcal{K}, d \in \mathcal{D}, \\
\sum_{\ell \in \mathcal{L}} \sum_{k \in \mathcal{K}} a_{n \ell} x_{\ell k}^{(d)}=s_{n}^{(d)}, & n \in \mathcal{N} \backslash d, d \in \mathcal{D}, \\
q_{\ell k} \geq 0, & \ell \in \mathcal{L}, k \in \mathcal{K}, \\
\sum_{k \in \mathcal{K}} \sum_{\ell \in \mathcal{O}(n)} q_{\ell k} \sum_{\ell^{\prime} \in \mathcal{L}} \tilde{\gamma}_{\ell \ell \prime}^{(k)} \leq P_{n}, & n \in \mathcal{N}, \\
\sum_{d \in \mathcal{D}} x_{\ell k}^{(d)}+\sum_{\ell^{\prime} \in \mathcal{L} \backslash\{\ell\}} \tilde{\gamma}_{\ell \ell^{\prime}}^{(k)} \log \left(1+q_{\ell^{\prime} k} g_{\ell \ell^{\prime}}^{(k)}\right) \leq \alpha S_{\ell k}, & \ell \in \mathcal{L}, k \in \mathcal{K},
\end{array}
$$

where $S_{\ell k}=\tilde{\gamma}_{\ell}^{(k)} \log \left(1+p_{\ell k}^{(0)} g_{\ell \ell}^{(k)}\right)+\sum_{\ell^{\prime} \in \mathcal{L}} \tilde{\gamma}_{\ell^{\prime}}^{(k)} \log \left(1+p_{\ell^{\prime} k}^{(0)} g_{\ell \ell^{\prime}}^{(k)}\right)$ is the fixed noisy-signal part.

Now, if we attempt to solve the optimization problem in (8.3), since the optimizer tends to maximize the data rates which appears in the first summation in LHS 
of $(8.3 \mathrm{~g})$, it will automatically push all the powers to zero so that the rates in the LHS jump to their boundaries. Having all powers equal to zero is not a desired outcome. To avoid this outcome, we have to introduce a lower bound on the powers to prevent the optimizer from pushing powers to zero. To do this, we use the approximation in [11]. This approximation is based on high SINR assumption and complies to the GP framework.

Having introduced the lower bound, the interference sub-problem can be rewritten in the following form:

$$
\max _{\left\{s_{n}^{(d)}\right\},\left\{x_{\ell k}^{(d)}\right\},\left\{q_{\ell k}\right\}} \sum_{d \in \mathcal{D}} \sum_{n \in \mathcal{N} \backslash\{d\}} w_{n}^{(d)} s_{n}^{(d)},
$$

subject to

$$
\begin{array}{ll}
s_{n}^{(d)} \geq 0, & n \in \mathcal{N} \backslash d, d \in \mathcal{D}, \\
x_{\ell k}^{(d)} \geq 0, & \ell \in \mathcal{L}, k \in \mathcal{K}, d \in \mathcal{D}, \\
\sum_{\ell \in \mathcal{L}} \sum_{k \in \mathcal{K}} a_{n \ell} x_{\ell k}^{(d)}=s_{n}^{(d)}, & n \in \mathcal{N} \backslash d, d \in \mathcal{D}, \\
q_{\ell k} \geq 0, & \\
\sum_{k \in \mathcal{K}} \sum_{\ell \in \mathcal{O}(n)} q_{\ell k} \sum_{\ell^{\prime} \in \mathcal{L}} \tilde{\gamma}_{\ell \ell^{\prime}}^{(k)} \leq P_{n}, & n \in \mathcal{L}, k \in \mathcal{K}, \\
\sum_{d \in \mathcal{D}} x_{\ell k}^{(d)}+\sum_{\ell^{\prime} \in \mathcal{L} \backslash\{\ell\}} \tilde{\gamma}_{\ell \ell^{\prime}}^{(k)} \log \left(1+q_{\ell^{\prime} k} g_{\ell \ell^{\prime}}^{(k)}\right) \leq \alpha S_{\ell k}, & \ell \in \mathcal{L}, k \in \mathcal{K}, \\
B_{\ell k} \leq \sum_{\ell^{\prime} \in \mathcal{L} \backslash\{\ell\}} \tilde{\gamma}_{\ell \ell^{\prime}}^{(k)} \log \left(q_{\ell^{\prime} k} g_{\ell \ell^{\prime}}^{(k)}\right), & \\
& \ell \in \mathcal{L}, k \in \mathcal{K},
\end{array}
$$

where $B_{\ell k}=\sum_{\ell^{\prime} \in \mathcal{L} \backslash\{\ell\}} \tilde{\gamma}_{\ell \ell^{\prime}}^{(k)} \log \left(p_{\ell^{\prime} k}^{(0)} g_{\ell \ell^{\prime}}^{(k)}\right)$ is the lower bound.

The interference sub-problem in (8.4) is amenable to GP framework. In particular, using logarithmic change of variables, one can rewrite (8.4) in a form that conforms into GP framework [8]. GP is known to be efficiently solvable as it can be readily convert into convex optimization [9].

\subsubsection{Noisy-signal Sub-problem}

In this section we consider the complementary case of the one that we considered in the previous section, i.e., the case when interference part in LHS of (8.2) is fixed. Again, we assume that an initial power allocation, $\left\{p_{\ell k}^{(0)}\right\}$, is given. Analogous to the 
discussion in the previous section, for the noisy-signal sub-problem we introduce a parameter $\beta \leq 1$ to control the search region for the power allocation around the given initial point.

Having introduced the parameter $\beta$, the noisy-signal sub-problem can be expresses as

$$
\max _{\left\{s_{n}^{(d)}\right\},\left\{x_{\ell k}^{(d)}\right\},\left\{q_{\ell k}\right\}} \sum_{d \in \mathcal{D}} \sum_{n \in \mathcal{N} \backslash\{d\}} w_{n}^{(d)} s_{n}^{(d)}
$$

subject to

$$
\begin{array}{lr}
s_{n}^{(d)} \geq 0, & n \in \mathcal{N} \backslash d, d \in \mathcal{D}, \\
x_{\ell k}^{(d)} \geq 0, & \ell \in \mathcal{L}, k \in \mathcal{K}, d \in \mathcal{D}, \\
\sum_{\ell \in \mathcal{L}} \sum_{k \in \mathcal{K}} a_{n \ell} x_{\ell k}^{(d)}=s_{n}^{(d)}, & n \in \mathcal{N} \backslash d, d \in \mathcal{D}, \\
q_{\ell k} \geq 0, & \ell \in \mathcal{L}, k \in \mathcal{K}, \\
\sum_{k \in \mathcal{K}} \sum_{\ell \in \mathcal{O}(n)} q_{\ell k} \sum_{\ell^{\prime} \in \mathcal{L}} \tilde{\gamma}_{\ell \ell \ell}^{(k)} \leq P_{n}, & n \in \mathcal{N}, \\
\sum_{d \in \mathcal{D}} x_{\ell k}^{(d)}+\beta I_{\ell k} \leq \tilde{\gamma}_{\ell}^{(k)} \log \left(1+q_{\ell k} g_{\ell \ell}^{(k)}\right)+\sum_{\ell^{\prime} \in \mathcal{L}} \tilde{\gamma}_{\ell \ell^{\prime}}^{(k)} \log \left(1+q_{\ell^{\prime} k} g_{\ell \ell^{\prime}}^{(k)}\right), \\
& \ell \in \mathcal{L}, k \in \mathcal{K},
\end{array}
$$

where $I_{\ell k}=\sum_{\ell^{\prime} \in \mathcal{L} \backslash\{\ell\}} \tilde{\gamma}_{\ell \ell^{\prime}}^{(k)} \log \left(1+p_{\ell^{\prime} k}^{(0)} g_{\ell \ell^{\prime}}^{(k)}\right)$ is the fixed interference part.

Note that, although in this case the optimizer tends to push the powers as high as possible to maximize the rates, there is no need for an upper bound on the powers. The reason is that powers are already bounded by the budget constraint in (8.5f). In other words, powers cannot go beyond nodes' budgets. The problem in (8.5) is convex and hence, it can be solved optimally with highly efficient IPM-based solvers.

\subsubsection{Iterative Solution for Power Allocation Sub-problem}

In this section, we develop an iterative technique that incorporates the convex problems explained in Section 8.4.2.1 to solve the problem in (8.1) when schedules are fixed.

Starting from a feasible initial power allocation, we first solve the interference sub-problem in (8.4) for a value of $\alpha>1$. The solution then will be used as an initial point for the signal sub-problem in (8.5) with a value of $\beta<1$. The output of this 
sub-problem is then used as an initial point for the subsequent iteration. In order for this technique to converge, as iterations go on, we need to expand the feasible region less such that at convergence, the outputs of both noisy-signal and interference subproblems become equal. To achieve this goal, we adjust parameters $\alpha$ and $\beta$ at each iteration accordingly. In particular, we set $\alpha_{i+1} \leq \alpha_{i}$ and $\beta_{i+1} \geq \beta_{i}$ where $i$ represents the $i$ th iteration. At convergence, we must have $\alpha^{*}=\beta^{*}=1$. It is worth noting that the step size taken by $\alpha$ and $\beta$ must be chosen such that they are not too small as it may causes the algorithm to converge slowly and not too large as it may cause the two sub-problems to over-cross each other. This algorithm is summarized in Algorithm 1.

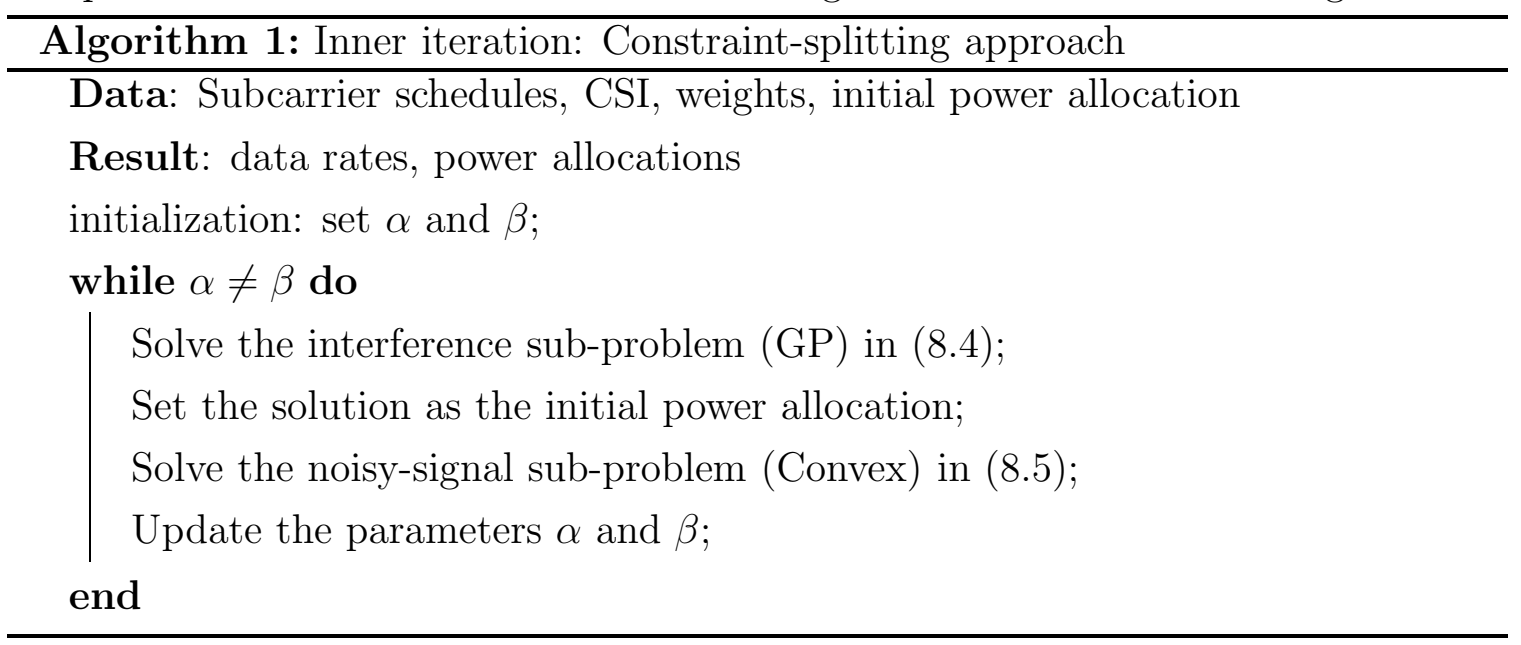

\subsection{Proposed Low Complexity Approach}

In the previous section, we considered the joint optimization problem when either the schedules or the powers are fixed. Using the techniques developed in Sections 8.4.1 and 8.4.2, in this section, we solve the entire optimization problem in (8.1). Our approach is composed of two stages, one for solving the joint optimization problem with fixed powers, and one for solving it with fixed schedules. Iterating between these two stages yields a stationary point which is an approximated solution for the joint design problem. It is worth noting that in contrast to the GP-based approach in Chapter 7, this algorithm has much less computational complexity as it needs fewer iterations for convergence, cf. Section 8.6 below.

In this algorithm we begin from a feasible initial point for which we choose equal distribution of budget among outgoing links, i.e., $p_{\ell k}^{(0)}=\frac{P_{n}}{K|\mathcal{O}(n)|}, n=1, \cdots, N$. This initial point gives all the links in the network the opportunity to contribute in routing. In the first stage, we fix the power allocation in (8.1) to $\left\{q_{\ell k}^{(0)}\right\}$ and then we solve 
the remaining LP problem to find the optimal schedules. Let $\left\{\widetilde{c}_{\ell \ell^{\prime}}^{(k)}\right\}$ be the values of scheduling variables at the output. In the second stage, by fixing the schedules in (8.1) to $\left\{\widetilde{c}_{\ell \ell^{\prime}}^{(k)}\right\}$, we use Algorithm 1 to find the power allocations. Let $\widetilde{p}_{\ell k}$ be that power allocation. This power allocation can be fed back into the first stage to solve the problem iteratively. However, our numerical results suggest that this outer iteration in its current form provides negligible performance gain. The reason is that in practice schedules and powers are directly related, i.e., if one of the powers is zero, the corresponding schedules are also zero and vice versa. Hence, feeding the current output into the first stage, yields almost the same schedules usually provides a negligible performance gain. To circumvent this difficulty, we need to modify the power allocations such that it enables us to explore the feasible region. To modify the output of the second stage, we use the gradient method [8] to find the gradient ascent direction of the problem in (8.1). To compute this gradient, we incorporate the inequality constraints in (8.1) in the objective. To do that, we use the log-barrier method [8]. In particular, we rewrite the problem in (8.1) in the following form:

$$
\begin{aligned}
\max \sum_{n, d} w_{n}^{(d)} s_{n}^{(d)} & +\frac{1}{t}\left(\sum_{n, d} \log \left(s_{n}^{(d)}\right)+\sum_{\ell, k, d} \log \left(x_{\ell k}^{(d)}\right)+\sum_{\ell, k} \log \left(\gamma_{\ell \ell^{\prime}}^{(k)}\right)+\sum_{\ell, k} \log \left(q_{\ell k}\right)\right) \\
& +\frac{1}{t} \sum_{k} \log \left(1-\sum_{\ell \ell^{\prime} \in \mathcal{L}} \gamma_{\ell \ell^{\prime}}^{(k)}\right)+\frac{1}{t} \sum_{n} \log (\phi(n))+\frac{1}{t} \sum_{\ell, k} \log (\psi(\ell, k)),
\end{aligned}
$$

subject to

$$
\sum_{\ell, k} a_{n \ell} x_{\ell k}^{(d)}=s_{n}^{(d)}, \quad n \in \mathcal{N} \backslash d, d \in \mathcal{D} .
$$

where $\phi(n) \triangleq P_{n}-\sum_{k, \ell \in \mathcal{O}(n)} q_{\ell k} \sum_{\ell^{\prime}} \gamma_{\ell \ell^{\prime}}^{(k)}, \quad \psi(\ell, k) \triangleq \gamma_{\ell \ell}^{(k)} \log \left(1+q_{\ell k} g_{\ell \ell}^{(k)}\right)+$ $\sum_{\ell^{\prime}} \gamma_{\ell \ell^{\prime}}^{(k)} \log \left(1+\frac{q_{\ell k} g_{\ell \ell}^{(k)}}{1+q_{\ell^{\prime} k} g_{\ell \ell^{\prime}}^{(k)}}\right)-\sum_{d} x_{\ell k}^{(d)}$ and $t$ is the log-barrier parameter. Note that $\phi(n)$ and $\psi(\ell, k)$ represent the gap between the RHS and LHS of the constraints in $(8.1 \mathrm{~h})$ and $(8.1 \mathrm{i})$, respectively. The gradient of the optimization problem in (8.6) with respect to $q_{\ell k}$ can be readily shown to be given by

$$
\nabla_{q_{\ell k}}=\frac{1}{t}\left(\frac{1}{q_{\ell k}}-\frac{\sum_{\ell \in \mathcal{L}_{+}(n), k, \ell^{\prime}} \gamma_{\ell \ell^{\prime}}}{\phi(n)}+\frac{\left.\frac{\gamma_{\ell \ell}^{(k)} g_{\ell \ell}^{(k)}}{1+q_{\ell k} g_{\ell \ell}^{(k)}}+\sum_{\ell^{\prime}} \frac{\gamma_{\ell \ell^{\prime}}^{(k)} g_{\ell \ell}^{(k)}\left(1+g_{\ell \ell^{\prime}}^{(k)}\left(q_{\ell^{\prime} k}-q_{\ell k}\right)\right)}{\left(1+q_{\ell^{\prime} k} g_{\ell \ell^{\prime}}^{(k)}\right)^{2}}\right)}{\psi(\ell, k)}\right)
$$


Setting $t=1$, we use the gradient ascent direction in (8.7) to update the output of the second stage and feed it back into the first stage at the subsequent iteration. In particular, we use the following update rule:

$$
q_{\ell k}^{(j+1)}=\tilde{q}_{\ell k}^{(j)}+\gamma_{j} \nabla_{\tilde{q}_{\ell k}}, \quad \ell \in \mathcal{L}, k \in \mathcal{K},
$$

where $j$ is the index of outer iterations and $\gamma_{j}$ is a step size. Iterations continue until a stopping criterion is satisfied, e.g., no more significant improvement in the objective is observed. This algorithm is summarized in Algorithm 2 and corresponding block diagram is illustrated in Figure 8.2.

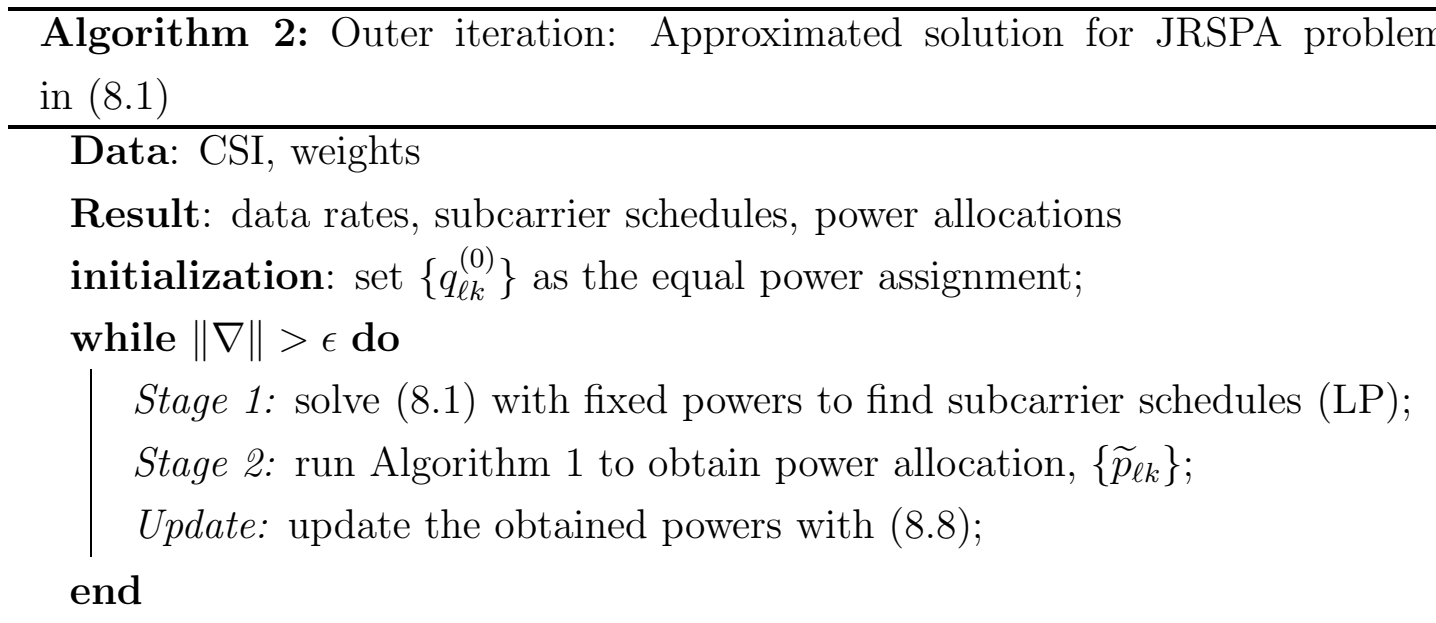

It will be shown in Section 8.7 that Algorithm 2 yields solutions that perform significantly better than those yielded by a fixed power allocation approaches. This algorithm will also be compared to the GP-based approach in Chapter 7 and it will be shown that this algorithm tends to yield a better performance with significantly less computational cost.

In the next section, we will provide bounds on the computational complexity of the proposed techniques. In particular, we will show that each stage of the algorithm has a polynomial complexity and hence, the proposed algorithm for obtaining an approximate solution to the joint optimization problem in (8.1) also has polynomial complexity. 


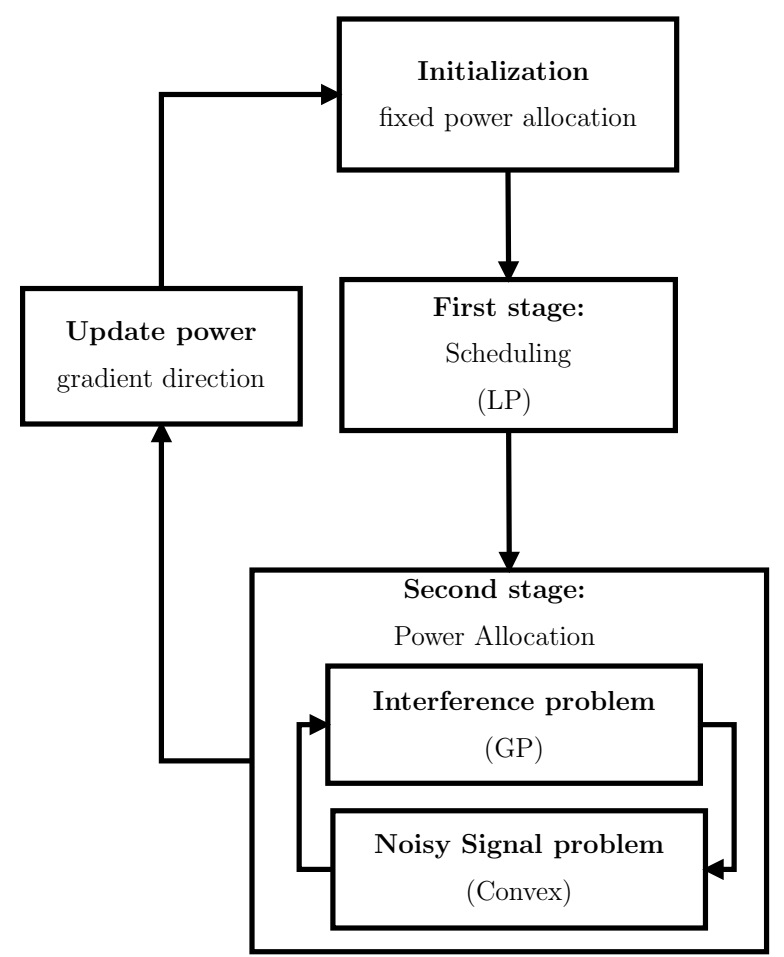

Figure 8.2: Block diagram of the proposed algorithm in Algorithm 2.

\subsection{Complexity Analysis}

The approach proposed in the previous section is based on iterating between two stages. In the first stage, we seek to find the optimal schedules for a given power allocation, whereas in the second stage, we find a suboptimal power allocation for a given schedules. The complexity of each stage is discussed next.

\subsubsection{Computational complexity of the first stage}

In the first stage of the approach proposed in the previous section, the power allocations are fixed. In this case, the problem in (8.1) breaks down into an LP where the optimal solution, i.e., optimal scheduling could be found efficiently IPM-based solvers. Using these solvers, the number of Newton iterations required to obtain the solution of LP can be shown to be proportional to $\sqrt{m}$, where $\mathrm{m}$ is the number of inequality constraints [8]. For the LP problem, we have $m=L K(D+1)+D(N-$ $1)+N+K+K N(N-1)\left(1+\frac{(N-2)^{2}}{2}\right)$. In addition, each Newton step is known to have a cubic complexity [70]. Hence, in the worst case scenario when network is fully connected, i.e., $L=N(N-1)$ and all the nodes are destination nodes, i.e., $D=N$, 
the computational complexity of solving the LP problem is $\mathcal{O}\left(\frac{1}{2} K^{3.5} N^{14}\right)$.

\subsubsection{Computational complexity of the second stage}

In the second stage, sub-optimal power allocations for given schedules are obtained by solving a sequence of convex problems. The complexity of each problem is discussed next.

\subsubsection{Computational complexity of the interference sub-problem}

The interference sub-problem discussed in Section 8.4.2.1 yields a GP which can be easily converted into a convex problem using the exponential change of variables [9]. The computational complexity of such problems were studied in the previous chapters. The essence of these studies is to bound the complexity of a GP problem by bounding each monomial term with a new variable that serves as an upper bound. Using this fact, it can be shown that the complexity of solving the GP problem in worst case scenario is $\mathcal{O}\left(4 K^{3.5} N^{14}\right)$.

\subsubsection{Computational complexity of the noisy-signal sub-problem}

The noisy-signal sub-problem discussed in Section 8.4.2.2 yields a convex optimization problem. Using a discussion analogous to the one in Section 8.6.1, it can be shown that the number of inequality constraints is $m=L K(D+2)+D(N-1)+N$. Hence, the complexity of solving this problem in the worst case scenario using IPMbased solvers is $\mathcal{O}\left(K^{3.5} N^{10.5}\right)$.

\subsubsection{Computational complexity of the two-stage approach}

We begin by recalling the definition of $\alpha$ and $\beta$ as the parameters used to control the search region for a power allocation solution. Let $\epsilon$ be the step size with which the parameter $\alpha$ shrinks at each inner iteration of the algorithm. In our analysis, we choose $\beta=\frac{1}{\alpha}$. Since at convergence, we must have $\alpha=1$, it implies that the number of iteration required for convergence is $\frac{\alpha}{\epsilon}$. Using the two-stage approach presented in Section 8.5 and the complexity discussions in Sections 8.6.2.1 and 8.6.2.2, it can be seen that the complexity of each outer iteration of the proposed approach is bounded 
by

$$
\mathcal{O}\left(K^{3.5} N^{10.5}\left(\frac{\alpha}{\epsilon}(4 N+1)\right)+\frac{1}{2}\right) .
$$

Note that the algorithm in Section 8.5 is based on solving a sequence of problems each with the complexity presented in (8.9). The length of this sequence depends on the choice of the step size, $\gamma_{j}$. If sequential step sizes form a monotonically decreasing

sequence and also they satisfy $\sum_{j} \gamma_{j}=\infty$, the gradient ascent method is guaranteed to converge [8].

It is worth noting that in Chapter 7, the complexity of solving the joint design optimization is exponential in $N$. For the reduced-size problem similar to the one we discussed here, it was shown in Chapter 7 that the complexity is bounded by $\mathcal{O}\left(K^{3.5} N^{14}\right)$. However, the number of iterations it takes for convergence is quite large which incurs a significant additional computational cost. For instance, in Chapter 7 it was shown that even for a small network of $N=4$ nodes, it took 180 iterations to converge. This is in contrast to the algorithm proposed herein, in which we are able to find a sub-optimal solution within a few iterations.

\subsection{Numerical Results}

In this section, we assess the performance of the iterative algorithm presented in Section 8.5. The solution of optimization problems in the numerical results reported herein are obtained using the software package CVX [62] with an underlying Mosek solver [64]. In our numerical results reported in this section, we choose $\gamma_{j}=\frac{0.2}{j}$.

The network is randomly generated by uniformly distributing the node positions within a cell with radius of $500 \mathrm{~m}$ and allowing two nodes to communicate if their distance is smaller than $150 \mathrm{~m}$ threshold. Such a network with $N=50$ nodes and $L=208$ links is illustrated in Figure 8.3. Among these nodes, 5 are randomly selected as source-destination nodes, labelled $D_{1}$ to $D_{5}$. The nodes are assumed to have identical power budgets, i.e, $P_{n}=P, n=1, \cdots, N$.

Recalling the channel model discussion in the second example of Section 4.6, we consider a standard communication channel model with quasi-static frequency-flat Rayleigh fading subcarriers, log-normal shadowing, and path loss components. As such, each complex subcarrier gain can be expressed as $h_{\ell k}=\sqrt{\rho(\ell) s_{\ell}} r_{\ell k}$, where $\rho(\cdot)$ is a path loss function, which depends on the propagation environment. Shadowing is 


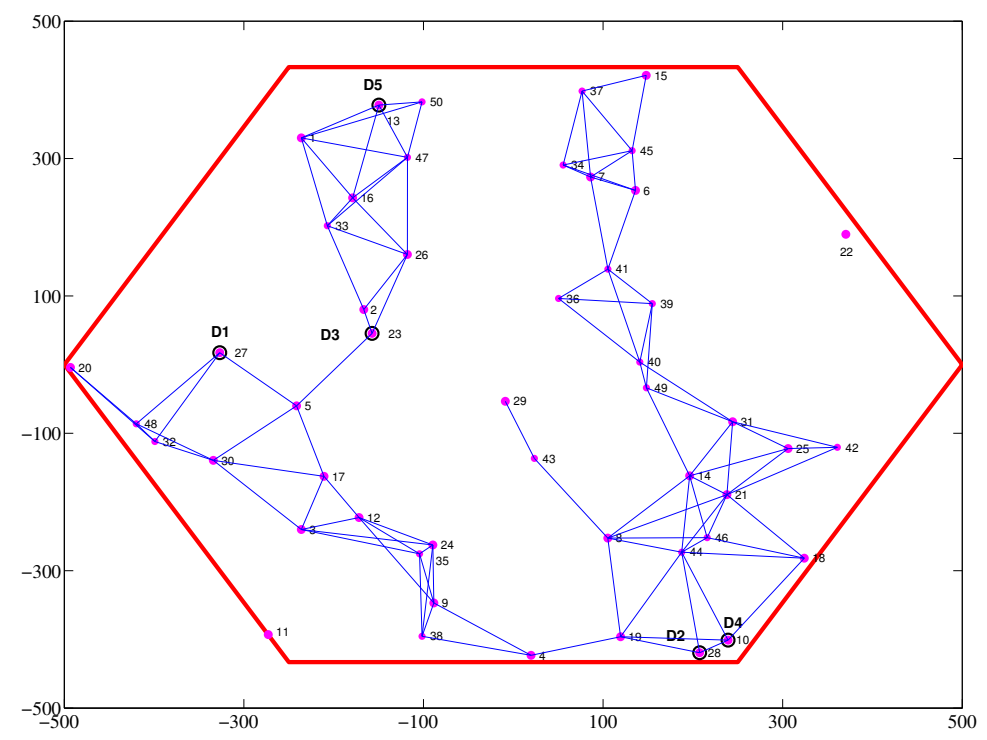

Figure 8.3: Network topology

represented by $s_{\ell}$, which is log-normal distributed with $0 \mathrm{~dB}$ mean and standard deviation $\sigma_{s}$ in $\mathrm{dB}$, and fading is represented by $r_{\ell k}$, which is complex Gaussian distributed with zero mean and unit variance. To simulate practical communication scenarios, we have selected the distance values and the log-normal shadowing and path loss parameters corresponding to the urban macro-cell (UMa) scenario of IMT-Advanced document [65]. For that scenario, the corresponding shadowing standard deviation, $\sigma_{s}$, is $6 \mathrm{~dB}$ and the noise power, $\sigma^{2}$, is chosen to be $174 \mathrm{dBm} / \mathrm{Hz}$. The path loss channel model is chosen to be the NLoS which by setting the carrier frequency to $2 \mathrm{GHz}$ and the elevation of each device to $1.5 \mathrm{~m}$ is expressed as $\rho(\ell)=10^{-18.66-40.32 \log _{10}\left(d_{\ell}\right)}$ where $d_{\ell}$ is the length of link $\ell$.

Example 1: (Performance Evaluation of the Proposed Scheme) In this example, we evaluate the performance of the scheme proposed in Section 8.5. To do that, consider the exemplary network in Figure 8.3. The available bandwidth is set to 10 MHz. The number of OFDM tones is 1024, which are assumed to be grouped into 16 subcarriers.

The average sum rates yielded by the algorithm in Section 8.5 for the values of $P$ ranging from 0 to $30 \mathrm{dBm}$ is depicted in Figure 8.4. The baseline scheme for comparison is the joint optimization without power allocation, i.e., the output of the first stage in Algorithm 2. Also Figure 8.4 provides a performance comparison with 


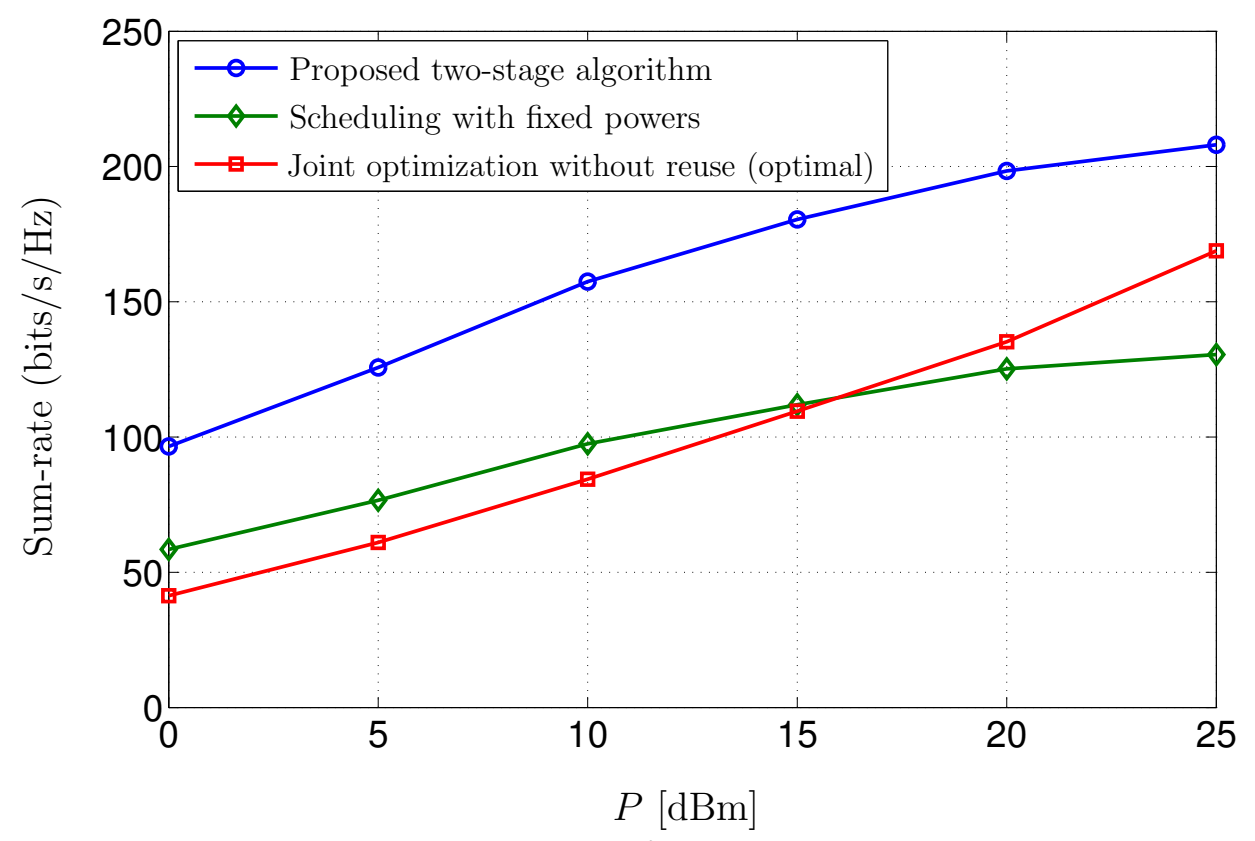

Figure 8.4: Performance evaluation

the design in which frequency-reuse is not considered, cf. Chapter 4.

As can be seen from Figure 8.4, the sum rate yielded by the proposed scheme significantly outperforms the design in which frequency-reuse is not considered. This figure also suggests that the power allocation technique in Section 8.4.2.3 and iterating over the two stages in Section 8.5 can improve the outcome of the scheduling technique significantly.

To illustrate the convergence behaviour of the power allocation technique in 8.4.2, in Figure 8.5 we depict the outputs of the interference and noisy-signal sub-problems in Section 8.4.2.1 versus the number of iterations. For this example we chose $\alpha_{i}=\frac{10}{i}$ and $\beta_{i}=\frac{1}{\alpha_{i}}$, where $i$ is the index of iteration. As shown in this figure, for the above example it takes only 10 iterations to converge which is quite fast compared to the algorithm in Chapter 7 which indicates the practicality of this algorithm for designing larger networks.

Example 2: (Performance Comparison with GP-based Approach) In this example, we compare the performance of our proposed algorithm in Section 8.5 with the one proposed in Chapter 7 to solve the same problem. The algorithm in Chapter 7 is based on approximating the constraints with monomial expressions and solving the 


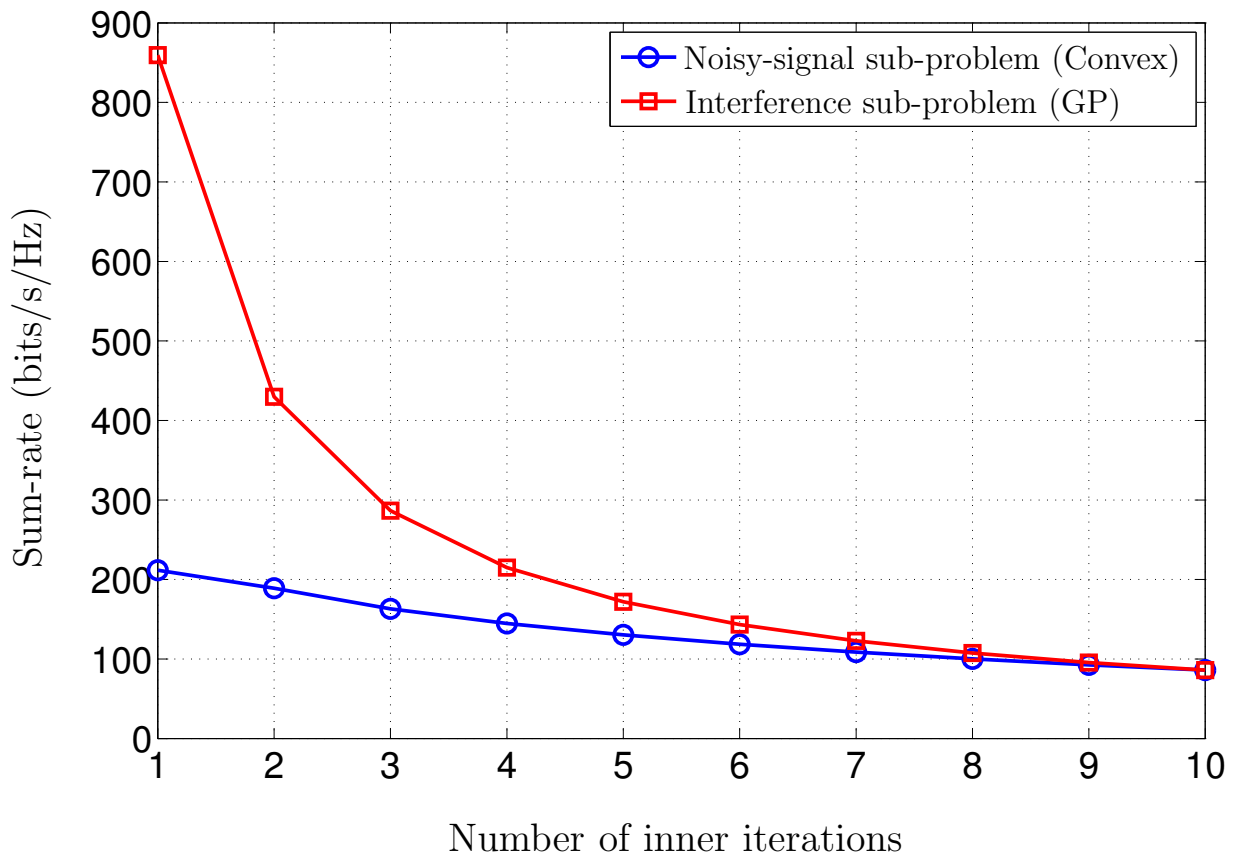

Figure 8.5: Convergence behaviour

approximated GP problem iteratively. This algorithm although analytically proved to converge to a KKT solution, has slow convergence. In particular, as shown in Chapter 7 for a small network of $N=4$ nodes, it takes 180 iterations to converge which makes it impractical for larger networks. Our proposed scheme in this chapter overcomes this difficulty. Indeed, the new scheme not only converges much faster than the algorithm in Chapter 7, but also offers better performance. This phenomenon can be attributed to the ability of the algorithm to find a better initial point. While the scheme in Chapter 7 starts from a random feasible initial point, our proposed scheme explores the feasible region for a better initial point by moving toward the gradient ascent direction. It is worth noting that as both the algorithms in Chapter 7 and Section 8.5 are suboptimal, the superior performance of one algorithm over the other one cannot be guaranteed.

To simulate the performance of these algorithms, consider an exemplary network with $N=3$ nodes and $L=6$ links depicted in Figure 8.1. We assume that there are $K=4$ subcarriers available, each with $200 \mathrm{KHz}$ bandwidth. In this network, two nodes wish to communicate with each other and the third node acts as a relay. The average sum rates yielded by the algorithm in Section 8.5 for the values of $P$ ranging from 0 to $30 \mathrm{dBm}$ are depicted in Figure 8.6. This figure also provides a comparison 


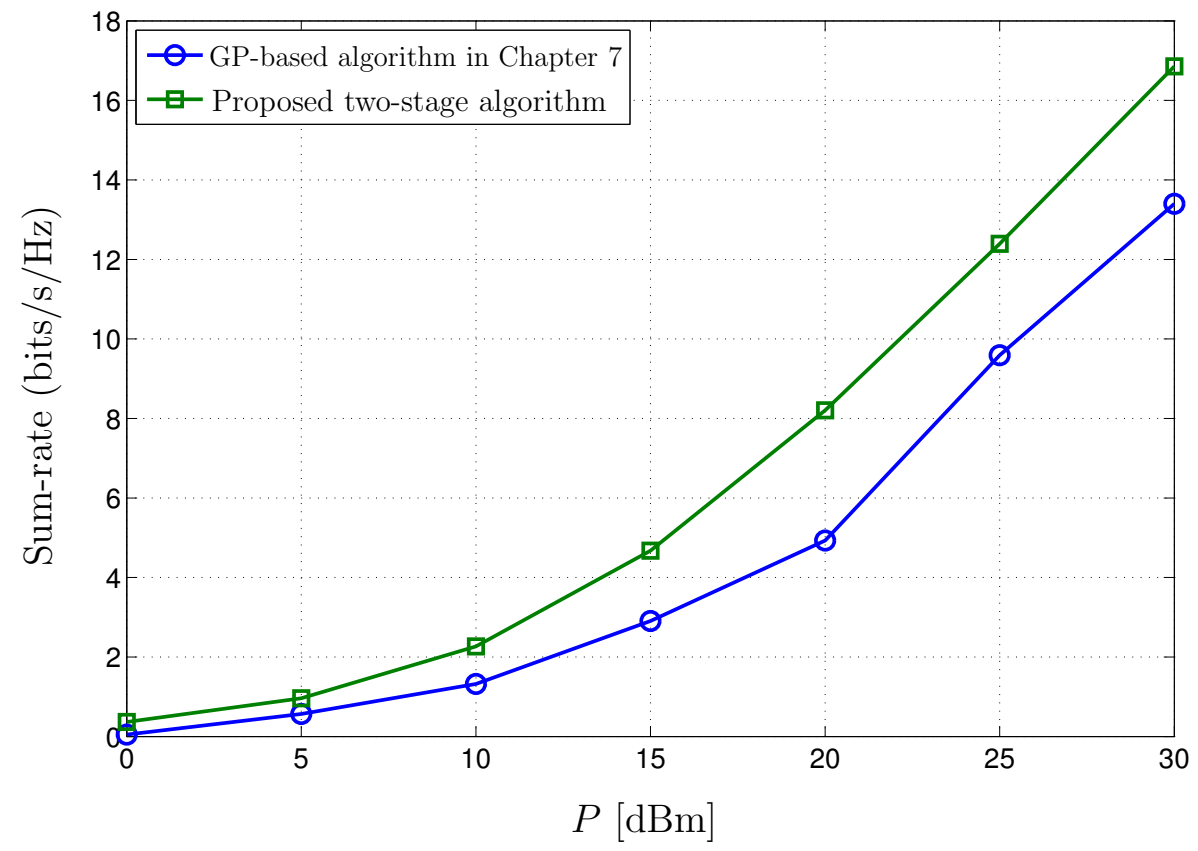

Figure 8.6: Performance comparison

with the sum rates yielded by the design based on GP-based approach in Chapter 7 .

As it can be seen from this figure, the algorithm proposed in this chapter offers higher sum-rates in comparison withe the GP-based approach in Chapter 7. It is worth noting that while our algorithm converges within 6 iterations, the one in Chapter 7 took 146 iterations to converge which renders it impractical for larger networks.

\subsection{Conclusion}

In this chapter we focused on the joint optimization of data routing, subcarrier scheduling and power allocations in a half-duplex multicarrier network when nodes can act as sources, destinations and relays. Our main contribution in this chapter is to develop a low-complexity algorithm for designing OFDMA networks such that it suits operation in medium-to-large networks. Our approach is based on decomposing the problem into two smaller but tractable stages, scheduling and power allocation, while allowing partial coupling between them. In the first stage, we solved the joint 
design when power allocations are fixed. This assumption yielded an LP which is known to be efficiently solvable. In the second stage, we solved the complementary design, i.e., the joint design when subcarrier schedules are fixed. This problem is nonconvex and difficult to solve. To overcome this difficulty, we proposed a novel iterative technique which we refer to as constraint-splitting approach. This approach exhibits fast convergence and finds a suboptimal power allocation in polynomial time. In this algorithm we split the capacity constraint into two parts. We defined appropriate lower and upper bounds and, at each iteration, we solved the problem by considering only one part while fixing the other one. It was shown that the corresponding problems are either GP or convex which are both efficiently solvable. By iterating over these parts and adjusting the bounds accordingly, we managed to find a suboptimal solution for the power allocation stage. To take advantage of these two sub-problems in solving the entire design problem, we developed an outer loop in which we used the gradient ascent direction to search for a good initial point. Using that initial point and the techniques developed for each of the sub-problems we managed to find an approximate solution for the OFDMA joint design problem. Our numerical results showed that the proposed algorithm has much less computational cost and offers higher performance gains in comparison with any currently available technique. 


\section{Chapter 9}

\section{Conclusions and Future Work}

\subsection{Summary and Contributions}

In this thesis, we focused on the joint design of data routes, subcarrier schedules and power allocations in a generic multicarrier wireless network. Each node was assumed to be capable of sending, receiving and relaying data simultaneously in the half-duplex operation mode. The work presented in this thesis opens new horizons for better exploitation of scarce resources in future wireless networks. The contributions of this thesis can be summarized as follows:

- We considered the case where each subcarrier is allowed to be used exclusively by one link at any time instant. In other words, subcarriers are allowed to be time-shared but not reused by multiple links in the network. In this case, by performing a change of variables, the joint optimization problem was cast in an efficiently solvable convex form. The computational complexity of this approach is polynomial in the number of nodes and subcarriers. Numerical results suggested that the joint design in this case offers significant performance improvements over the one with fixed power allocations.

- We studied the case where each subcarrier is used exclusively by one link during the whole signalling interval. In other words, subcarriers are neither time-shared nor reused by the links in the network. In this case, the joint design assumes a mixed integer form which is known to be NP-hard. To alleviate this difficulty, we developed two efficiently computable lower bounds on the maximum weighted sum of the rates that can be reliably communicated over this network. The first bound is based on a GP approximation of the original design problem, 
whereas the second bound is based on normalizing and rounding the solution of the problem in the first instance. We analyzed the computational complexity of these algorithms and it was found to be relatively small. Numerical investigations suggest that the gap between these lower bounds and the true optimal obtained through exhaustive search is generally small.

- We examined the case where a subcarrier, once assigned to a set of links, would be used by those links throughout the signalling interval. In other words, subcarriers were entitled to be potentially reused but not allowed to be time-shared by multiple links in the network. In this case, the joint design problem was shown to be nonconvex. To alleviate this difficulty, we developed an efficient iterative approach that enabled us to obtain locally optimal solutions in polynomial time. Although potentially suboptimal, for some network scenarios, the data routes and power allocations obtained by our technique enabled achieving significantly higher rates than those achieved by their optimal counterparts in scheduling-based cross layer designs.

- We considered the general case where a subcarrier can be used by a set of links at any time instant. In other words, subcarriers can be both time-shared and reused by multiple links in the network. Hence, this instance generalizes the aforementioned three instances and its performance constitutes an upper bound on their performance. Considering both time-sharing and frequencyreuse jointly gave rise to, generally difficult to solve, nonconvex optimization problems. To circumvent this difficulty, we invoked a GP-based approximation technique and provided a method for obtaining locally optimal solutions. Despite its potential performance gains, the proposed optimization framework is computationally complex and is thus only suitable for designing small-tomedium size networks. For larger networks, this framework can be seen as a benchmark and a first step towards developing practical designs that make judicious use of the degrees of design freedom offered by the physical wireless medium. Numerical results showed that the design developed in this instance yielded performance that is superior to that of their counterparts in which frequency-reuse and time-sharing are not considered simultaneously.

- We focused on the general case when subcarriers can be reused and time-shared 
by multiple links. Our main contribution in this case is to propose a lowcomplexity algorithm for designing large wireless OFDMA networks. Our approach relies on decomposing the problem into two smaller but tractable stages, i.e., scheduling and power allocation, while allowing partial coupling between them. In the first stage, we solve the joint design with the power allocations fixed. The design problem in this case reduces to an efficiently solvable LP. In the second stage, we solve the joint design with subcarrier schedules fixed. This problem is nonconvex and difficult to solve. To overcome this difficulty we proposed a novel iterative technique which we refer to as constraint-splitting. This technique exhibits fast convergence and finds a suboptimal power allocation in polynomial time. In this technique we split the capacity constraint into two parts. We observe that by fixing the RHS of this constraint and defining an appropriate lower bound, the problem can be cast as a GP, which can be readily converted into a convex optimization problem. We also observe that fixing the LHS of the constraint makes the problem convex and hence, efficiently solvable. To take advantage of the two sub-problems in solving the entire design problem, we developed an outer loop in which we use gradient ascent direction to search for a good initial point. Using that initial point and the techniques developed for each of the sub-problems we managed to find an approximated solution for the joint design problem. Our numerical results showed that the proposed algorithm has much less computational cost and offers higher performance gains in comparison with the GP-based technique.

\section{$9.2 \quad$ Future Work}

The work presented in this thesis opens new horizons for future research.

Distributed Designs: The design considered in this thesis is centralized, in the sense that the design is performed by a central entity that is aware of the network parameters and it then forwards the decisions to all the nodes, possibly over a dedicated control channel. With the rapid growth of wireless devices, future wireless networks are expected to be denser with larger numbers of nodes. For such networks, the signalling exchange between the nodes and the central entity might render the centralized designs less practical due to the excessive amount of signalling overhead. Hence, it is desirable to develop distributed or 
semi-distributed designs in which the central entity off-loads all or parts of the joint design to the nodes of the networks. Such designs could be implemented in less structured networks such as sensor networks.

Imperfect CSI: In this work perfect CSI was assumed available at the central entity. In other words we assumed that at the beginning of each scheduling interval, each node estimates its CSI and then sends this information to the central entity via a dedicated control channel. It is also assumed that this information is correctly received at the central entity. In real networks, these assumptions are not generally precise. First, CSI is usually estimated and second, CSI may erroneously be received at the central entity. Imperfect CSI may result in rates that cannot be reliably decoded. An interesting research problem is to take into account the impact of imperfect CSI in joint design of the network.

Delay Constraints: In this research, scarce radio resources are allocated in a way that optimizes objective measures based on delivered end-to-end rates. In this work we did not consider the number of hops involved in routing data between source-destination pairs. This can result in transmission delays which may not be tolerable for some users. Although increasing the weights of less delay tolerable users prioritize them over other users, it does not guarantee their delay requirements. An extension to our work is to reformulate the design problem such that it incorporates the delay constraints.

Data Traffic Models: In this research, we developed joint designs of routes, schedules and power allocations in networks with full-buffer traffic. An important extension of this work is to study the case of different traffic models with different burstiness levels. In such cases, the designs developed herein are not readily appropriate and hence it is important to devise fundamentally different designs that incorporate various traffic models.

Clustered Networks: In this research we proposed a low-complexity approach for designing large networks. For future wireless networks with massive connectivity and large number of devices, this approach can be improved further. In particular, the network can be decomposed into several clusters and within each cluster the algorithms proposed herein can be used for designing that cluster. Developing a framework for communication between the clusters and coordinating them through a central entity is currently underway. 
Resource-Block Granularity: In this work we considered the scheduling at the subcarrier level. In particular, when time-sharing is allowed, each subcarrier can be shared by multiple users with infinite granularity. Since in real systems, the signalling interval is divided into resource blocks, it has limited granularity and hence, from practical point of view, sharing subcarriers in its general form cannot be implemented. An interesting extension to this work is to consider the scheduling at the resource-block level rather than the subcarrier level. In this case the designs proposed herein constitute upper bounds on the performance of the systems with finite granularity.

Energy Efficiency: The objective of the designs developed in this thesis was to maximize a weighted sum of the rates that are communicated over the network. Another interesting objective that could be maximized is the energy-efficiency which can be interpreted as minimizing the power consumption in the network while satisfying the quality of service constraints of each user. This is specially interesting for designing future wireless networks where most of devices are battery-operated and energy efficiency is crucial for their communications. 


\section{Appendix A}

\section{Geometric Programming}

\section{A.1 The GP Standard Form}

A GP optimization problem can be readily transformed to an efficiently solvable convex one. To provide the standard form of a GP, let $z \in \mathbb{R}^{n}$ be a vector of positive

entries. A monomial in $z$ is defined to be a function of the form $c_{0} \prod_{i} z_{i}^{\alpha_{i}}$ and a posynomial in $z$ is defined to be a function of the form $\sum_{k} c_{k} \prod_{i} z_{i}^{\alpha_{i k}}$, where $c_{k}>0$, and $\left\{\alpha_{i}\right\}$ and $\left\{\alpha_{i k}\right\}$, are arbitrary constants, $k=0,1, \ldots$, and $i=1, \ldots, n$. A standard GP is an optimization problem of the following form with $\left\{f_{i}\right\}$ being posynomials and $\left\{g_{i}\right\}$ being monomials $[9,11]$ :

$$
\begin{aligned}
& \min _{z} f_{0}(z), \\
& \text { subject to } f_{i}(z) \leq 1, \quad i=1, \ldots, m \text {, } \\
& g_{i}(z)=1, \quad i=1, \ldots, p .
\end{aligned}
$$

\section{A.2 Monomial Approximation}

A monomial approximation of a differentiable function $h(z) \geq 0$ near $z^{(0)}$ is given by its first order Taylor expansion in the logarithmic domain [9]. Defining $\beta_{i}=$ $\left.\frac{z_{i}^{(0)}}{h\left(z^{(0)}\right)} \frac{\partial h}{\partial z_{i}}\right|_{z=z^{(0)}}$, we have

$$
h(z) \approx h\left(z^{(0)}\right) \prod_{i=1}^{n}\left(\frac{z_{i}}{z_{i}^{(0)}}\right)^{\beta_{i}} .
$$

This approximation is used to provide a GP approximation of the cross-layer design problem. 


\section{Appendix B}

\section{Single Condensation Method}

In GP-based approaches, the technique in Appendix A.2 is used to approximate each posynomial on the RHS of the inequality constraints in the GP-framework by a monomial. The solution of the GP resulting from this approximation is then used as an initial point for the next iteration and so on. The iterates generated by this sequential algorithm converge to a solution of the KKT system corresponding to the original problem if the conditions outlined in [11] and [10] are satisfied. For completeness, these conditions for the instance of the constraint in (5.12e) can be expressed as

1. $\delta_{\ell k}\left(q_{\ell k} / q_{\ell k}^{(0)}\right)^{\theta_{\ell k}} \leq W N_{0}+q_{\ell k}\left|h_{\ell k}\right|^{2}$,

2. $\left.\delta_{\ell k}\left(q_{\ell k} / q_{\ell k}^{(0)}\right)^{\theta_{\ell k}}\right|_{q_{\ell k}=q_{\ell k}^{(0)}}=1+\left.q_{\ell k}\left|h_{\ell k}\right|^{2}\right|_{q_{\ell k}=q_{\ell k}^{(0)}}$,

3. $\left.\frac{\partial\left(\delta_{\ell k}^{-1}\left(q_{\ell k}^{(0)} / q_{\ell k}\right)^{\theta} \ell k\right)}{\partial q_{\ell k}}\right|_{q_{\ell k}=q_{\ell k}^{*}}=\left.\frac{\partial\left(1+q_{\ell k}\left|h_{\ell k}\right|^{2}\right)^{-1}}{\partial q_{\ell k}}\right|_{q_{\ell k}=q_{\ell k}^{*}}$,

where at each iteration $\delta_{\ell k}=1+q_{\ell k}^{(0)}\left|h_{\ell k}\right|^{2}$, and $\theta_{\ell k}=\frac{q_{\ell k}^{(0)}\left|h_{\ell k}\right|^{2}}{\delta_{\ell k}}$. The optimal power allocation at convergence point is denoted by $q_{\ell k}^{*}$. These three conditions are known to be satisfied by affine functions (see e.g., [56]), which guarantees convergence of the single condensation method. 


\section{Appendix C}

\section{Complexity Analysis}

\section{C.1 Self-Concordance}

The complexity analysis of the algorithms proposed in this work hinges on the assumption that the log-barrier functions of the problems considered herein possess the self-concordant property which is defined as follows: We begin by recalling the definition of self-concordant functions [8].

Definition 1. (Self-concordance)

$A$ function $f: \mathbb{R}^{n} \rightarrow \mathbb{R}$ is said to be self-concordant if, for all $x, v \in \mathbb{R}^{n}, s \in \mathbb{R}$ such that $x+s v$ is in the domain of $f$ and $\left|\frac{\partial^{3}}{\partial s^{3}} f(x+s v)\right| \leq 2 \frac{\partial^{2}}{\partial s^{2}} f(x+s v)^{3 / 2}$.

\section{C.2 Proof of Proposition 1}

To bound the complexity of solving the problem (4.11), we begin by noting that its log-barrier function is not self-concordant. This difficulty can be circumvented by adding the following auxiliary set of constraints which have no effect on the actual feasible set or the final solution:

$$
c_{\ell k}+y_{\ell k} g_{\ell k} \geq 0, \quad \forall k \in \mathcal{K}, \ell \in \mathcal{L}
$$

To construct the log-barrier function, $\psi$, the sum of the logarithm of the inequality constraints in (4.11) and the auxiliary constraints in (C.1) is superimposed on the scaled objective in (4.11). In particular, using $t$ to denote the non-negative scalar 
that multiplies the objective of the log-barrier function of the IPM, we can write

$$
\begin{aligned}
\psi=\phi & -\sum_{\ell, k} \log \left(c_{\ell k} \log \left(1+\frac{y_{\ell k} g_{\ell k}}{c_{\ell k}}\right)-\sum_{d} \frac{x_{\ell k}^{(d)}}{W}\right)-\sum_{\ell, k} \log \left(c_{\ell k}+y_{\ell k} g_{\ell k}\right), \\
\phi= & -t \sum_{n} \sum_{d} w_{n}^{(d)} s_{n}^{(d)}-\sum_{\ell, k, d} \log \left(x_{\ell k}^{(d)}\right)-\sum_{n, d} \log \left(s_{n}^{(d)}\right)-\sum_{\ell, k} \log \left(c_{\ell k}\right) \\
& -\sum_{\ell, k} \log \left(y_{\ell k}\right)-\sum_{k} \log \left(1-\sum_{\ell} c_{\ell k}\right)-\sum_{n} \log \left(P_{n}-\sum_{\ell, k} y_{\ell k}\right) .
\end{aligned}
$$

The function $\phi$ represents the log-barrier function of a linear optimization problem, which is known to be self-concordant [8]. It remains to show the self-concordance of the last two terms of (C.2). To show this, we add and subtract $2 \sum_{\ell, k} \log c_{\ell k}$ to (C.2). Hence,

$$
\psi=\phi-2 \log \left(c_{\ell k}\right)-\sum_{\ell, k} \log \left(\log \left(1+\frac{y_{\ell k} g_{\ell k}}{c_{\ell k}}\right)-\frac{\sum_{d} x_{\ell k}^{(d)}}{W c_{\ell k}}\right)-\log \left(1+\frac{y_{\ell k} g_{\ell k}}{c_{\ell k}}\right) .
$$

Now, the first two terms of (C.3) are self-concordant. To show the self-concordance of the last two terms, we note that these terms can be expressed in the form $-\log b-$ $\log (\log b-a)$, where $a=\frac{\sum_{d} x_{\ell k}^{(d)}}{W c_{\ell k}}$ and $b=1+\frac{y_{\ell k} g_{\ell k}}{c_{\ell k}}$. This form is known to be selfconcordant [8], which establishes the fact that the log-barrier function corresponding to the problem in (4.11) is self-concordant. We will now use this result to bound the complexity of the IPM that solves (4.11).

With the log-barrier function possessing the self-concordance property, the number of Newton iterations required to obtain the solution of (4.11) can be shown to be proportional to $\sqrt{m}$ where $m$ is the number of inequality constraints. In addition, each Newton step is known to have a cubic complexity [70]. Hence the computational complexity of finding the optimal continuous scheduling-based design in (4.11) can be bounded by $\mathcal{O}\left((L K(4+D)+N+K+D(N-1))^{3.5}\right)$, which completes the proof of the first statement of Proposition 1. 


\section{C.3 Proof of Proposition 2}

\section{C.3.1 Proving the First Statement of Proposition 2}

To prove the first statement of Proposition 2, we note that the rounding-based lower bound requires solving (4.11) followed by solving (5.4) with the corresponding fixed rounded schedules.

To bound the complexity of solving (5.4), we show that the log-barrier function corresponding to this problem is self-concordant. To do so, we add the following auxiliary set of constraints:

$$
1+\frac{p_{\ell k}\left|h_{\ell k}^{2}\right|}{W N_{0}} \geq 0, \quad \forall \ell \in \mathcal{L}, k \in \mathcal{K} .
$$

Invoking these constraints and arguing along the same lines as in the proof of the first statement, it can be shown that the capacity constraints in (5.4e) can be cast in the self-concordant form. Using this result, the complexity of solving (5.4) with IPM can be shown to be bounded by $\mathcal{O}\left((L K(3+D)+N+D(N-1))^{3.5}\right)$. Details are omitted for brevity.

\section{C.3.2 Proving the Second Statement of Proposition 2}

To determine the complexity of solving the problem in (5.12), we begin by converting this problem into a convex one, using standard exponential transformations as follows:

$$
\begin{array}{lrl}
t_{n}^{(d)}=\exp \left(\ln (2) s_{n}^{(d)}\right), & n \in \mathcal{N} \backslash\{d\}, d \in \mathcal{D}, \\
r_{\ell k}^{(d)}=\exp \left(\ln (2) \frac{x_{\ell k}^{(d)}}{W}\right), & \ell \in \mathcal{L}, k \in \mathcal{K}, d \in \mathcal{D}, \\
y_{\ell k}=\exp \left(q_{\ell k}\right), & \ell \in \mathcal{L}, k \in \mathcal{K} .
\end{array}
$$

Substituting the variables in (5.12) with the ones in (C.5) and taking the logarithm of the obtained objective and constraints result in a convex optimization problem which can be solved efficiently using IPM technique. To use this technique, a logbarrier function is synthesized from the objective and inequality constraints. The complexity analysis of the IPM technique is simplified when the log-barrier function is self-concordant [8], cf., Definition 1. 
The log-barrier function corresponding to the convex form of (5.12) can be expressed as

$$
\phi=-t \sum_{n} \sum_{d} w_{n}^{(d)} s_{n}^{(d)}+\psi,
$$

where $\psi$ represents the component of the log-barrier function associated with the inequality constraints in the convex form of (5.12). To examine whether $\phi$ is selfconcordant, we note that the converted objective in $(5.12 \mathrm{a})$ and the inequality constraints corresponding to $(5.12 \mathrm{c}),(5.12 \mathrm{~d})$ and $(5.12 \mathrm{~g})$ are linear and therefore their corresponding components in the log-barrier function are self-concordant [8]. Hence it remains to consider the self-concordance for the constraints in (5.12e) and (5.12f). For simplicity, we write the posynomial constraint in (5.12e) in the standard form in (A.1). After changing the variables and taking the logarithm of both sides, this constraint can be written in a general form as

$$
\log \left(\sum_{i} \exp \left(a_{i} \alpha_{i}+b_{i} \beta_{i}+c_{i}\right)\right) \leq 0
$$

where $\left\{\alpha_{i}\right\},\left\{\beta_{i}\right\}$ are the optimization variables and $\left\{a_{i}\right\},\left\{b_{i}\right\},\left\{c_{i}\right\}$ are constants. The component corresponding to the constraint in (C.7) in the log-barrier function can now be expressed as

$$
-\log \left(-\log \sum_{i} \exp \left(a_{i} \alpha_{i}+b_{i} \beta_{i}+c_{i}\right)\right)
$$

To ensure that (C.8) is self-concordant, we introduce auxiliary variables, $\lambda_{i}$, to bound the exponentially transformed variables in (C.7). Using these new variables, the constraint in (C.7) can be replaced with the following set of constraints [8]:

$$
\begin{aligned}
& \sum_{i} \lambda_{i} \leq 1, \\
& \lambda_{i} \geq 0, \\
& a_{i} \alpha_{i}+b_{i} \beta_{i}+c_{i}-\log \lambda_{i} \leq 0 .
\end{aligned}
$$

Now the associated log-barrier function of the constraints in (C.9) can be shown to be self-concordant, cf., [8, Example 9.8].

By introducing new auxiliary variables, we construct a self-concordant log-barrier function. Using this function, the complexity can be shown to be proportional to 
$m^{3.5}$, where $m$ is the number of inequality constraints. Hence, the complexity of solving (5.12) can be bounded by $\mathcal{O}\left((L K(3 L+2)+N+D(N-1))^{3.5}\right)$, which completes the proof of the first statement of Proposition 2.

\section{C.3.3 Proving the Third Statement of Proposition 2}

The optimal solution of (5.2) can be found by using exhaustive search over all possible binary schedules. Each feasible schedule, satisfying (4.9h), corresponds to a situation in which each subchannel is either not used by any of the $L$ links or used by one of them. Since there are $K$ subchannels, the number of possible schedules to search over is $(L+1)^{K}$. For each schedule, a convex optimization problem similar to the one in (5.4) is solved using IPM, which implies that the overall complexity of solving the JRSPA problem in (5.2) is bounded by $\mathcal{O}\left((L+1)^{K}(L K(3+D)+N+\right.$ $\left.D(N-1))^{3.5}\right)$. This completes the proof of the last statement of Proposition 2.

\section{C.4 Proof of Proposition 3}

Each iteration of the GP-based approach involves solving a GP of the form in (6.8). Following the steps analogous to the ones described in Appendix C.3, such a GP is converted to a convex problem using a standard exponential change of variables. Using auxiliary variables to bound the exponentially transformed variables, the logbarrier function corresponding to (6.8) can be shown to be self-concordant [8]. Using this result, the complexity of solving the GP in (6.8) can be shown to be bounded by $\mathcal{O}\left((L K(3+D+(L-1) / 2)+N+D(N-1))^{3.5}\right)$. See [8] for more details.

\section{C.5 Proof of Proposition 4}

The proof of the Proposition 4 follows by using an argument similar to the one used in the proof of the second statement of Proposition 2 and is omitted for brevity.

After constructing a self-concordant log-barrier function, it can be verified that the number of inequality constraints in (8.4) is equal to $2 L K N+N+D(N-1)+2 K\left(2^{L}-1\right)$, from which Proposition 4 follows. 


\section{List of References}

[1] "Cisco visual networking index: Forecast and methodology, 2014-2019," white paper, Cisco Systems Inc., May 2015. Available at: http://http: //www.cisco.com/c/en/us/solutions/collateral/service-provider/ ip-ngn-ip-next-generation-network/white_paper_c11-481360.pdf.

[2] J. G. Andrews, S. Buzzi, W. Choi, S. V. Hanly, A. Lozano, A. C. Soong, and J. C. Zhang, "What will 5G be?," IEEE J. Select. Areas Commun., vol. 32, pp. 1065-1082, June 2014.

[3] R. Pabst, B. Walke, D. Schultz, P. Herhold, H. Yanikomeroglu, S. Mukherjee, H. Viswanathan, M. Lott, W. Zirwas, M. Dohler, H. Aghvami, D. Falconer, and G. Fettweis, "Relay-based deployment concepts for wireless and mobile broadband radio," IEEE Commun. Mag., vol. 42, pp. 80-89, Sept. 2004.

[4] H. Yanikomeroglu, "Fixed and mobile relaying technologies for cellular networks," in Proc. 2nd Wksp. Apps Svcs. Wireless Ntwk, pp. 75-81, July 2002.

[5] M. N. Tehrani, M. Uysal, and H. Yanikomeroglu, "Device-to-device communication in 5G cellular networks: challenges, solutions, and future directions," IEEE Commun. Mag., vol. 52, no. 5, pp. 86-92, 2014.

[6] J. Leonard and J. Cimini, "Analysis and simulation of a digital mobile channel using orthogonal frequency division multiplexing," IEEE Trans. Commun., vol. 33, pp. 665-675, 1985.

[7] S. Sadr, A. Anpalagan, and K. Raahemifar, "Radio resource allocation algorithms for the downlink of multiuser OFDM communication systems," IEEE Commun. Surv. Tutorial, vol. 11, pp. 92-106, Aug. 2009.

[8] S. Boyd and L. Vandenberghe, Convex Optimization. Cambridge, UK: Cambridge University Press, 2004.

[9] S. Boyd, S.-J. Kim, L. Vandenberghe, and A. Hassibi, "A tutorial on geometric programming," Optimization and Engineering, vol. 8, pp. 67-127, Mar. 2007.

[10] B. R. Marks and G. P. Wright, "A general inner approximation algorithm for nonconvex mathematical programs," Operations Research, vol. 26, pp. 681-683, Aug. 1978. 
[11] M. Chiang, C. W. Tan, D. P. Palomar, D. O'Neil, and D. Julian, "Power control by geometric programming," IEEE Trans. Wireless Commun., vol. 6, pp. 26402650, July 2007.

[12] L. Liu, R. Zhang, and K.-C. Chua, "Achieving global optimality for weighted sum-rate maximization in the K-user Gaussian interference channel with multiple antennas," IEEE Trans. Wireless Commun., vol. 11, pp. 2640-2650, May 2012.

[13] S. R. Bhaskaran, S. V. Hanly, N. Badruddin, and J. S. Evans, "Maximizing the sum rate in symmetric networks of interfering links," Information Theory, IEEE Transactions on, vol. 56, pp. 4471-4487, Sept. 2010.

[14] A. Gjendemsj, D. Gesbert, G. E. Oien, and S. G. Kiani, "Binary power control for sum rate maximization over multiple interfering links," IEEE Trans. Wireless Commun., vol. 7, pp. 3164-3173, Aug. 2008.

[15] D. Gesbert, S. G. Kiani, A. Gjendemsjo, et al., "Adaptation, coordination, and distributed resource allocation in interference-limited wireless networks," IEEE Proc., vol. 95, pp. 2393-2409, Dec. 2007.

[16] D. Julian, M. Chiang, D. O’Neill, and S. Boyd, "QoS and fairness constrained convex optimization of resource allocation for wireless cellular and ad hoc networks," in Proc. IEEE Int. Conf. Comp. Commun. (INFOCOM), vol. 2, pp. 477486, June 2002.

[17] H. Inaltekin and S. V. Hanly, "Optimality of binary power control for the single cell uplink," IEEE Trans. Inf. Theory, vol. 58, pp. 6484-6498, Oct. 2012.

[18] G. Song and Y. Li, "Utility-based joint physical-MAC layer optimization in OFDM," in Proc. IEEE Glob. Commun. Conf. (GLOBECOM), vol. 1, pp. 671675, Nov. 2002.

[19] J. Jang and K. B. Lee, "Transmit power adaptation for multiuser OFDM systems," IEEE J. Select. Areas Commun., vol. 21, pp. 171-178, Feb. 2003.

[20] G. Song and Y. Li, "Cross-layer optimization for OFDM wireless networks-part i: theoretical framework," IEEE Trans. Wireless Commun., vol. 4, pp. 614-624, Mar. 2005.

[21] Z. Shen, J. G. Andrews, and B. L. Evans, "Adaptive resource allocation in multiuser OFDM systems with proportional rate constraints," IEEE Trans. Wireless Commun., vol. 4, pp. 2726-2737, Nov. 2005.

[22] A. Eryilmaz and R. Srikant, "Fair resource allocation in wireless networks using queue-length-based scheduling and congestion control," in Proc. IEEE Int. Conf. Comp. Commun. (INFOCOM), vol. 3, pp. 1794-1803, Mar. 2005.

[23] G. Song and Y. Li, "Utility-based resource allocation and scheduling in OFDMbased wireless broadband networks," IEEE Commun. Mag., vol. 43, pp. 127-134, Dec. 2005. 
[24] H. Li and H. Liu, "An analysis of uplink OFDMA optimality," IEEE Trans. Wireless Commun., vol. 6, pp. 2972-2983, Aug. 2007.

[25] K. Kim, Y. Han, and S.-L. Kim, "Joint subcarrier and power allocation in uplink OFDMA systems," IEEE Commun. Lett., vol. 9, pp. 526-528, June 2005.

[26] C. Y. Ng and C. W. Sung, "Low complexity subcarrier and power allocation for utility maximization in uplink OFDMA systems," IEEE Trans. Wireless Commun., vol. 7, pp. 1667-1675, May 2008.

[27] J. Huang, V. G. Subramanian, R. Agrawal, and R. Berry, "Joint scheduling and resource allocation in uplink OFDM systems for broadband wireless access networks," IEEE J. Select. Areas Commun., vol. 27, pp. 226-234, Feb. 2009.

[28] J. Huang, V. G. Subramanian, R. Agrawal, and R. A. Berry, "Downlink scheduling and resource allocation for OFDM systems," IEEE Trans. Wireless Commun., vol. 8, pp. 288-296, Jan. 2009.

[29] C. Y. Wong, R. S. Cheng, K. B. Lataief, and R. D. Murch, "Multiuser OFDM with adaptive subcarrier, bit, and power allocation," IEEE J. Select. Areas Commun., vol. 17, pp. 1747-1758, Oct. 1999.

[30] C. Y. Wong, R. S. Cheng, K. B. Letaief, and R. D. Murch, "Multiuser subcarrier allocation for ofdm transmission using adaptive modulation," in Proc. IEEE Vehicular Tech. Conf. (VTC), vol. 1, pp. 479-483, July 1999.

[31] R. Aggarwal, M. Assaad, C. E. Koksal, and P. Schniter, "Joint scheduling and resource allocation in the OFDMA downlink: Utility maximization under imperfect channel-state information," IEEE Trans. Signal Processing, vol. 59, pp. 55895604, Nov. 2011.

[32] C. W. Tan, S. Friedland, and S. H. Low, "Spectrum management in multiuser cognitive wireless networks: Optimality and algorithm," IEEE J. Select. Areas Commun., vol. 29, pp. 421-430, Feb. 2011.

[33] G. Li and H. Liu, "Downlink radio resource allocation for multi-cell OFDMA system," IEEE Trans. Wireless Commun., vol. 5, pp. 3451-3459, Dec. 2006.

[34] L. Venturino, N. Prasad, and X. Wang, "A successive convex approximation algorithm for weighted sum-rate maximization in downlink OFDMA networks," in Proc. IEEE Conf. Inf. Sci. Sys., pp. 379-384, Mar. 2008.

[35] M. Salem, A. Adinoyi, M. Rahman, H. Yanikomeroglu, D. Falconer, Y.-D. Kim, E. Kim, and Y.-C. Cheong, "An overview of radio resource management in relayenhanced OFDMA-based networks," IEEE Commun. Surv. Tutorial, vol. 12, no. 3, pp. 422-438, 2010.

[36] Y. Park and E.-S. Jung, "Resource-aware routing algorithms for multi-hop cellular networks," in Proc. Int. Conf. Multimedia and Ubiquitous Eng. (MUE), pp. 1164-1167, Apr. 2007. 
[37] G. Middleton and B. Aazhang, "Relay selection for joint scheduling, routing and power allocation in multiflow wireless networks," in 4th Int. Symp. Commun. Control, Signal Processing (ISCCSP), pp. 1-4, Mar. 2010.

[38] S. Sharma, Y. Shi, Y. T. Hou, H. D. Sherali, and S. Kompella, "Cooperative communications in multi-hop wireless networks: Joint flow routing and relay node assignment," in Proc. IEEE Int. Conf. Comp. Commun. (INFOCOM), pp. 1-9, Mar. 2010.

[39] M. Salem, A. Adinoyi, M. Rahman, and H. Yanikomeroglu, "Fairness-aware radio resource management in downlink OFDMA cellular relay networks," IEEE Trans. Wireless Commun., vol. 9, pp. 1628-1639, May 2010.

[40] M. Salem, A. Adinoyi, H. Yanikomeroglu, and D. Falconer, "Opportunities and challenges in ofdma-based cellular relay networks: A radio resource management perspective," Proc. IEEE Vehicular Tech. Conf. (VTC), vol. 59, pp. 2496-2510, June 2010.

[41] M. Salem, A. Adinoyi, H. Yanikomeroglu, and D. Falconer, "Fair resource allocation toward ubiquitous coverage in ofdma-based cellular relay networks with asymmetric traffic," IEEE Trans. Vehicular Tech., vol. 60, pp. 2280-2292, June 2011.

[42] M. Salem, A. Adinoyi, H. Yanikomeroglu, and Y.-D. Kim, "Radio resource management in OFDMA-based cellular networks enhanced with fixed and nomadic relays," in Proc. IEEE Wireless Commun. Ntwk Conf. (WCNC), pp. 1-6, Apr. 2010 .

[43] M. Kaneko and P. Popovski, "Radio resource allocation algorithm for relay-aided cellular OFDMA system," in Proc. IEEE Int. Conf. Commun. (ICC), pp. 48314836, June 2007.

[44] G. Li and H. Liu, "Resource allocation for OFDMA relay networks with fairness constraints," IEEE J. Select. Areas Commun., vol. 24, pp. 2061-2069, Nov. 2006.

[45] M. Tao and Y. Liu, "A network flow approach to throughput maximization in cooperative OFDMA networks," IEEE Trans. Wireless Commun., vol. 12, pp. 1138-1148, Mar. 2013.

[46] T. C.-Y. Ng and W. Yu, "Joint optimization of relay strategies and resource allocations in cooperative cellular networks," IEEE J. Select. Areas Commun., vol. 25, pp. 328-339, Feb. 2007.

[47] K. Hosseini and R. Adve, "Cooperative strategies and fairness-aware resource allocation in selection-based OFDM networks," in Proc. IEEE Int. Conf. Commun. (ICC), pp. 1-6, June 2011.

[48] K. Karakayali, J. Kang, M. Kodialam, and K. Balachandran, "Cross-layer optimization for OFDMA-based wireless mesh backhaul networks," in Proc. IEEE Wireless Commun. Ntwk Conf. (WCNC), pp. 276-281, Mar. 2007. 
[49] K.-D. Lee and V. C. Leung, "Fair allocation of subcarrier and power in an ofdma wireless mesh network," IEEE J. Select. Areas Commun., vol. 24, pp. 2051-2060, Nov. 2006.

[50] D. Incebacak, B. Tavli, , and H. Yanikomeroglu, "Trade-offs in sum-rate maximization and fairness in relay-enhanced OFDMA-based cellular networks," in Proc. IEEE Glob. Commun. Conf. (GLOBECOM), pp. 4770-4775, Dec. 2014.

[51] L. Le and E. Hossain, "Multihop cellular networks: Potential gains, research challenges, and a resource allocation framework," IEEE Commun. Mag., vol. 45, pp. 66-73, Sept. 2007.

[52] A. Nosratinia, T. E. Hunter, and A. Hedayat, "Cooperative communication in wireless networks," IEEE Commun. Mag., vol. 42, pp. 74-80, Oct. 2004.

[53] S. H. Ali, K.-D. Lee, and V. C. Leung, "Dynamic resource allocation in OFDMA wireless metropolitan area networks [radio resource management and protocol engineering for IEEE 802.16]," IEEE Wireless Commun. Mag., vol. 14, pp. 6-13, Feb. 2007.

[54] L. Xiao, M. Johansson, and S. P. Boyd, "Simultaneous routing and resource allocation via dual decomposition," IEEE Trans. Commun., vol. 52, pp. 11361144, July 2004.

[55] M. Johansson and L. Xiao, "Cross-layer optimization of wireless networks using nonlinear column generation," IEEE Trans. Wireless Commun., vol. 5, pp. 435445, Feb. 2006.

[56] R. H. Gohary and T. J. Willink, "Joint routing and resource allocation via superposition coding for wireless data networks," IEEE Trans. Signal Processing, vol. 58, pp. 6387-6399, 2010.

[57] R. H. Gohary and T. N. Davidson, "On power allocation for parallel gaussian broadcast channels with common information," EURASIP Wireless Commun. Ntwk., pp. 1-15, Feb. 2009.

[58] S. Shabdanov, P. Mitran, and C. Rosenberg, "Cross-layer optimization using advanced physical layer techniques in wireless mesh networks," IEEE Trans. Wireless Commun., vol. 11, pp. 1622-1631, 2012.

[59] A. Maaref, J. Ma, M. Salem, H. Baligh, and K. Zarifi, "Device-centric radio access virtualization for $5 \mathrm{G}$ networks," in Proc. IEEE Glob. Commun. Wkshp., pp. 887-893, Dec. 2014.

[60] X. Lin, N. B. Shroff, and R. Srikant, "A tutorial on cross-layer optimization in wireless networks," IEEE J. Select. Areas Commun., vol. 24, pp. 1452-1463, Aug. 2006.

[61] Y. Nesterov, A. Nemirovskii, and Y. Ye, Interior-Point Polynomial Algorithms in Convex Programming. Philadelphia, USA: SIAM, 1994. 
[62] M. Grant and S. Boyd, CVX: Matlab Software for Disciplined Convex Programming, version 1.21, Jan. 2011. Available at: http://cvxr.com/cvx.

[63] J.F.Sturm, "Using SeDumi 1.02, a Matlab toolbox for optimization over symmetric cones," Optim. Methods Softw., vol. 11-12, pp. 625-653, 1998.

[64] MOSEK Apps., The MOSEK Optimization Toolbox for Matlab Manual, Version 6.0, 2012. Available at: http://www.docs.mosek.com.

[65] Int. Telecommun. Union (ITU), Guidelines for Evaluation of Radio Interface Technologies for IMT-Advanced. ITU-R: TR M.21351, Dec. 2009. Available at: http://www.itu.int/pub/R-REP-M.2135-1-2009.

[66] C. W. Tan, "Optimal power control in rayleigh-fading heterogeneous networks," in Proc. IEEE Int. Conf. Comp. Commun. (INFOCOM), pp. 2552-2560, 2011.

[67] C. W. Tan, M. Chiang, and R. Srikant, "Maximizing sum rate and minimizing MSE on multiuser downlink: Optimality, fast algorithms and equivalence via max-min SINR," IEEE Trans. Signal Processing, vol. 59, no. 12, pp. 6127-6143, 2011.

[68] M. Charafeddine and A. Paulraj, "Sequential geometric programming for $2 \times 2$ interference channel power control," in Proc. IEEE Conf. Inf. Sci. Sys. , pp. 185189, Mar. 2007.

[69] C. W. Tan, D. P. Palomar, and M. Chiang, "Solving nonconvex power control problems in wireless networks: low SIR regime and distributed algorithms," in Proc. IEEE Glob. Commun. Conf. (GLOBECOM), vol. 6, pp. 3445-3450, Dec. 2005.

[70] G. Strang, Introduction to Linear Algebra. Wellesley, MA: Wellesley-Cambridge Press, 2009. 